\title{
A Systems Approach to Rich User Experience Design
}

by

\author{
Sara Fortier
}

A thesis submitted to the Faculty of Graduate and Postdoctoral Affairs in partial fulfillment of the requirements for the degree of

Master of Design

in

Industrial Design

Carleton University

Ottawa, Ontario

(C) 2012, Sara Fortier 
Library and Archives

Canada

Published Heritage

Branch

395 Wellington Street

Ottawa ON K1A ON4

Canada
Bibliothèque et

Archives Canada

Direction du

Patrimoine de l'édition

395 , rue Wellington

Ottawa ON K1A ON4

Canada
Your file Votre référence

ISBN: 978-0-494-93651-1

Our file Notre référence

ISBN: 978-0-494-93651-1
NOTICE:

The author has granted a nonexclusive license allowing Library and Archives Canada to reproduce, publish, archive, preserve, conserve, communicate to the public by telecommunication or on the Internet, loan, distrbute and sell theses worldwide, for commercial or noncommercial purposes, in microform, paper, electronic and/or any other formats.

The author retains copyright ownership and moral rights in this thesis. Neither the thesis nor substantial extracts from it may be printed or otherwise reproduced without the author's permission.
AVIS:

L'auteur a accordé une licence non exclusive permettant à la Bibliothèque et Archives Canada de reproduire, publier, archiver, sauvegarder, conserver, transmettre au public par télécommunication ou par l'Internet, prêter, distribuer et vendre des thèses partout dans le monde, à des fins commerciales ou autres, sur support microforme, papier, électronique et/ou autres formats.

L'auteur conserve la propriété du droit d'auteur et des droits moraux qui protege cette thèse. $\mathrm{Ni}$ la thèse ni des extraits substantiels de celle-ci ne doivent être imprimés ou autrement reproduits sans son autorisation.
In compliance with the Canadian Privacy Act some supporting forms may have been removed from this thesis.

While these forms may be included in the document page count, their removal does not represent any loss of content from the thesis.
Conformément à la loi canadienne sur la protection de la vie privée, quelques formulaires secondaires ont été enlevés de cette thèse.

Bien que ces formulaires aient inclus dans la pagination, il n'y aura aucun contenu manquant. 


\begin{abstract}
Today's saturated market of goods and services has created a new competitive battleground where companies are shifting to compete on the basis of providing compelling and memorable experiences for their users and customers. However, as the world grows in complexity, the designers responsible for delivering these rich experiences must address the sizeable networks of interconnected people, things, and environments surrounding the products and services they create. As such, design and research processes must evolve to embrace experience design through a system's lens. Using data collected from seven case studies of experience-centric design firms, including IDEO and Adaptive Path, this study explores the methodologies and organizational behaviours leading to the creation of rich user and customer experience from a system's perspective. From the findings five patterns particularly critical to designing for experience were uncovered: They include a holistic perspective, building an emotional connection, interdisciplinary collaboration, harmonizing touchpoints and client empowerment. A synthesis of these patterns led to the development of a Rich Experience Design Diffusion Approach (REDDA).
\end{abstract}




\section{ACKNOWLEDGEMENTS}

First and foremost, I would like to thank my advisor Prof. WonJoon Chung at the School of Industrial Design and my co-advisor Prof. Nicolas Papadopoulos from the Sprott School of Business for their continued support and guidance throughout this process, as well as the countless hours spent discussing and reviewing ideas.

Furthermore, I would like to thank Jennifer and Arjun for their invaluable and unrelenting efforts in connecting me with all the right people. I am also grateful to the designers, researchers, strategists, and leaders in experience design who participated in the interviews and made this study possible.

To all my friends, family, and classmates, thank you for helping me brainstorm when work seemed at a standstill and thank you for all of your encouragement. Lastly, I would like to say a special thank you to my parents for their undying love and support; they are and always will be my best cheerleaders. 


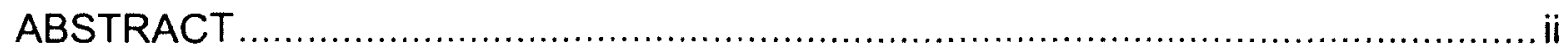

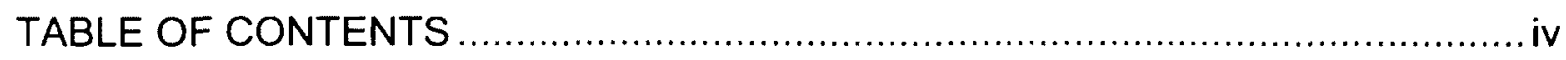

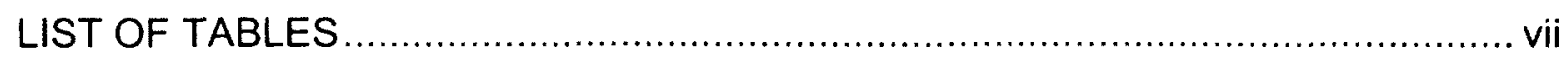

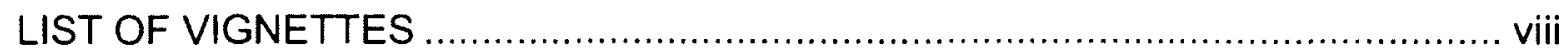

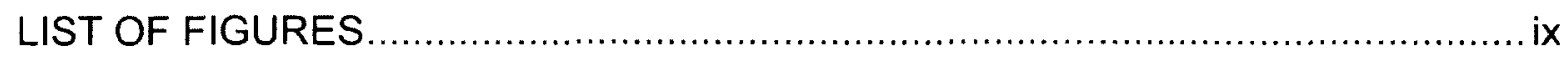

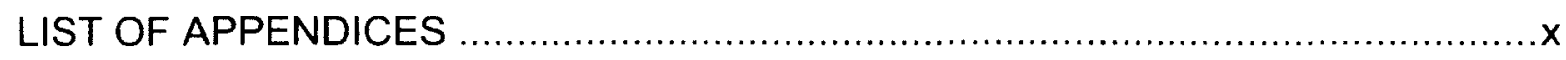

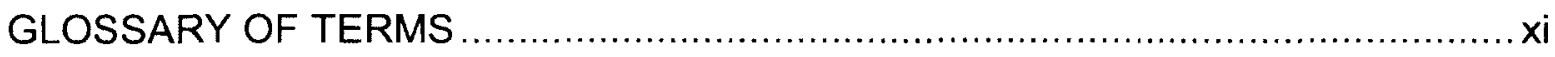

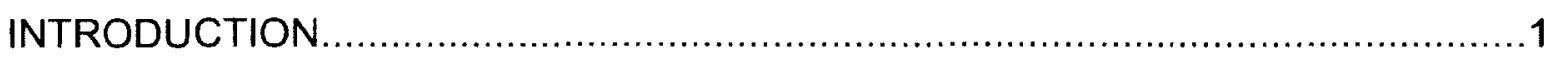

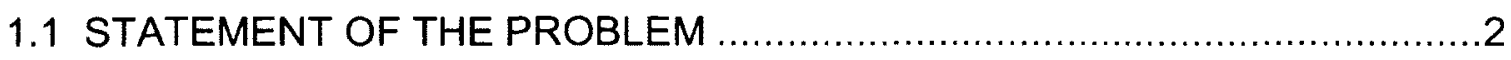

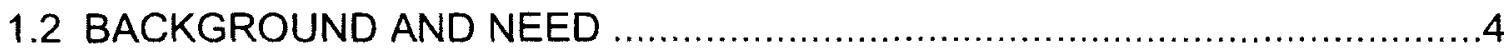

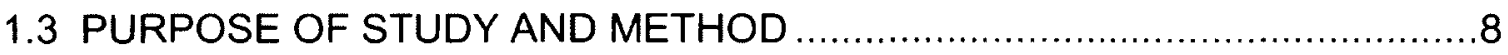

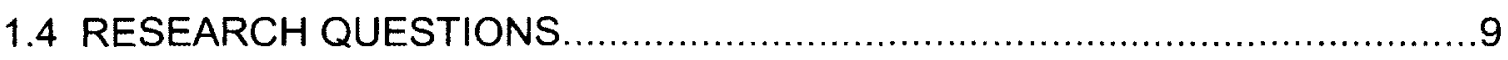

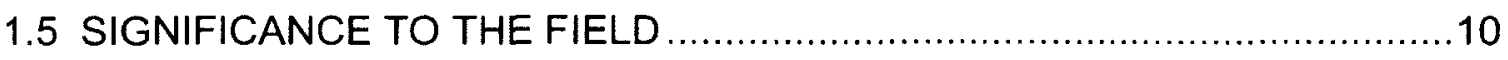

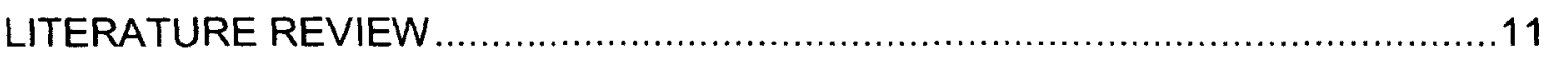

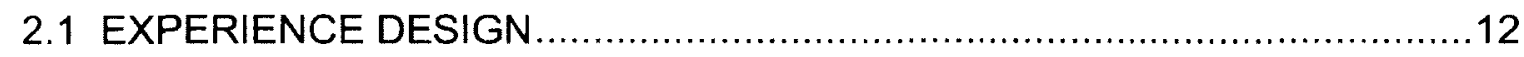

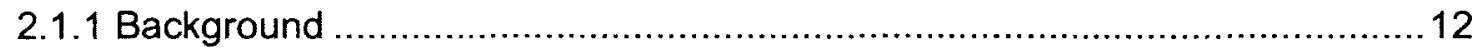

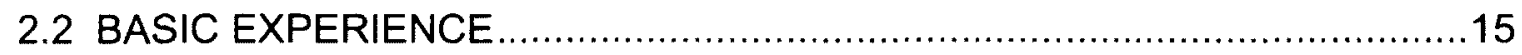

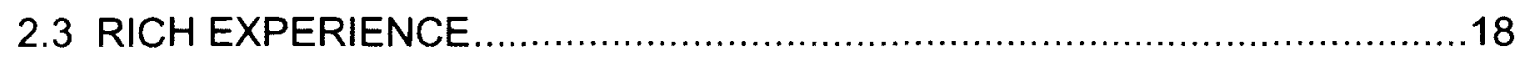

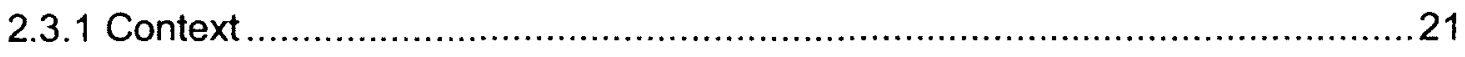

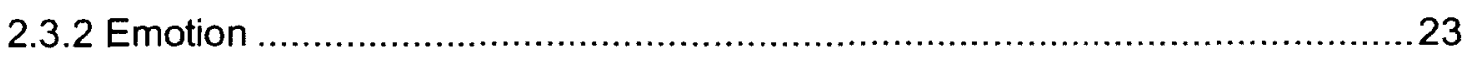

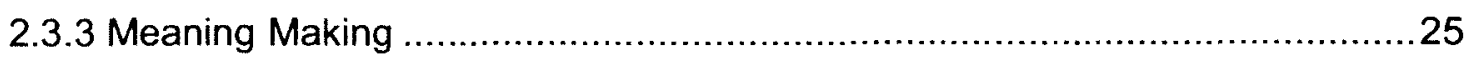

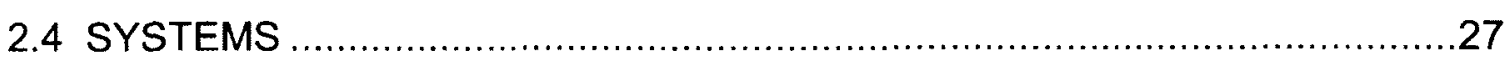




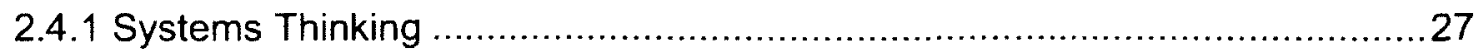

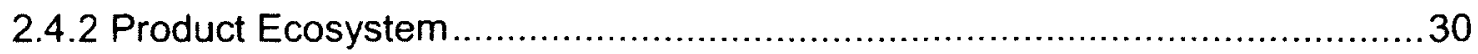

2.4.3 Product Ecosystem as a Design Strategy ..................................................

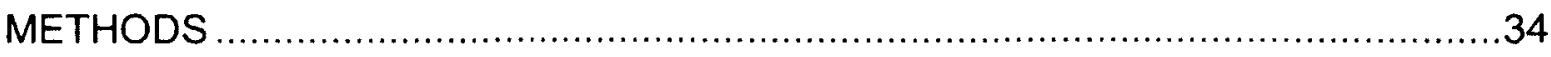

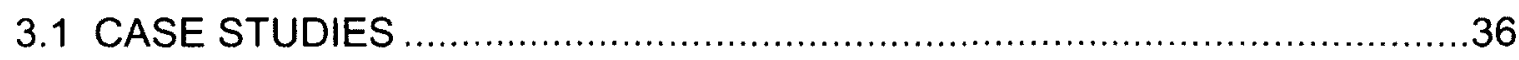

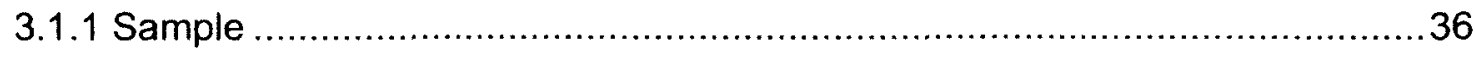

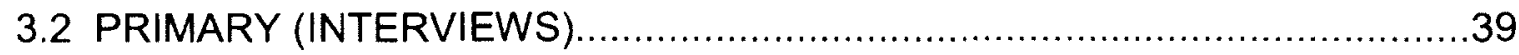

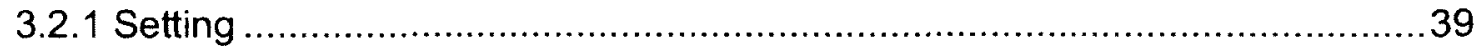

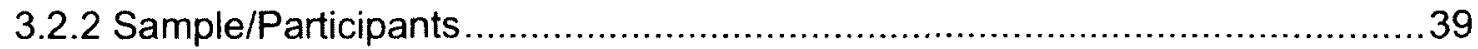

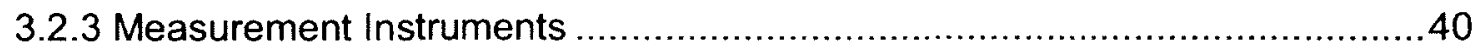

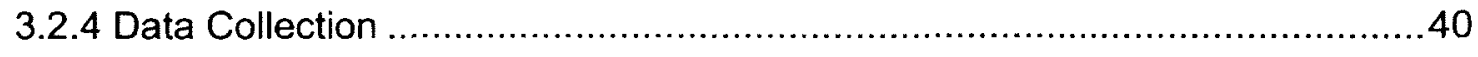

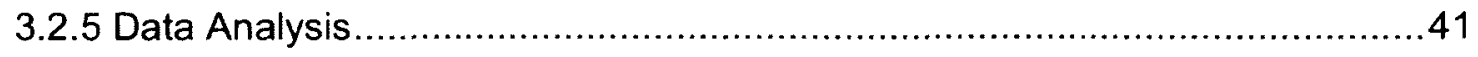

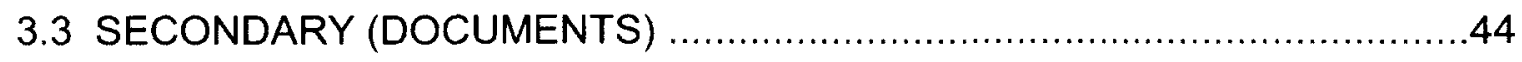

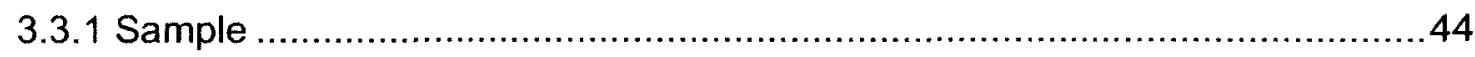

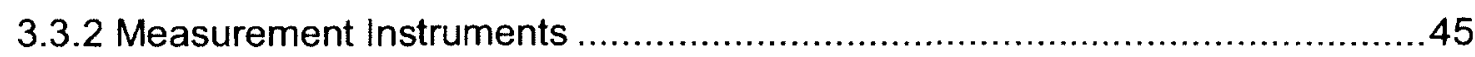

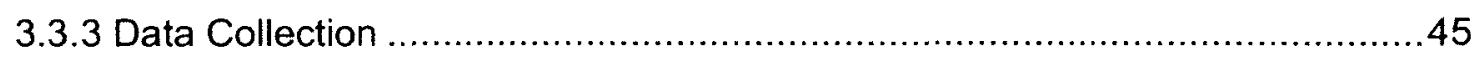

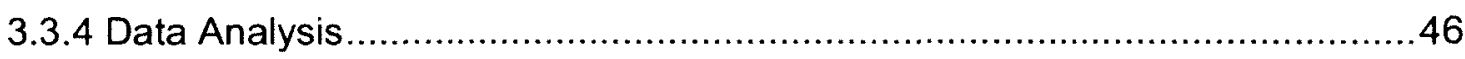

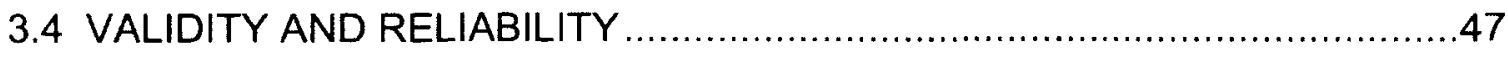

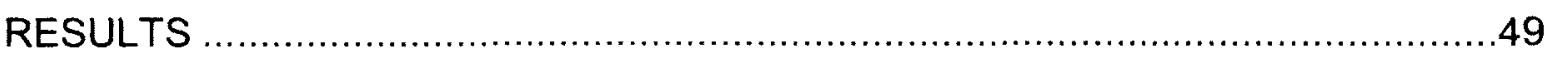

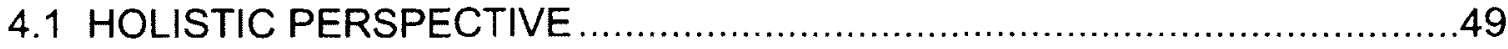

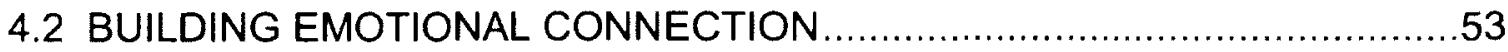

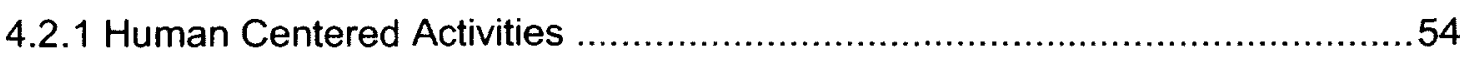

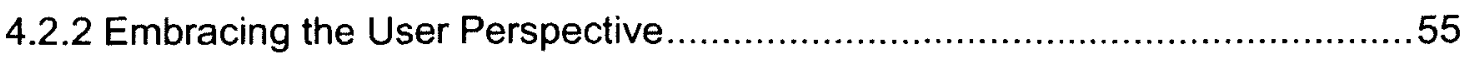

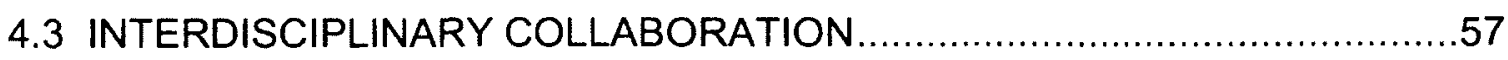

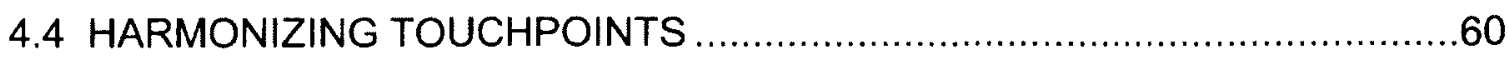




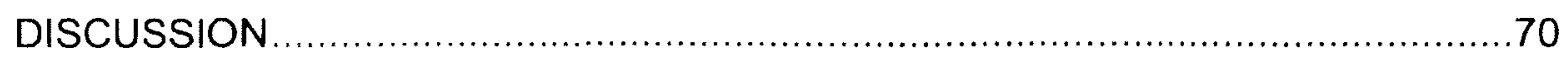

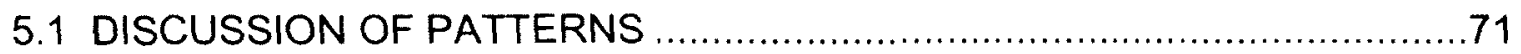

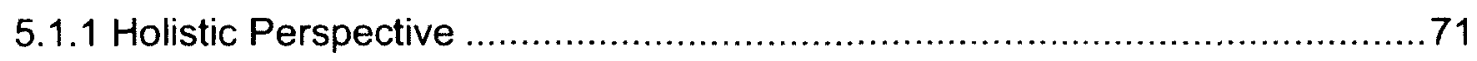

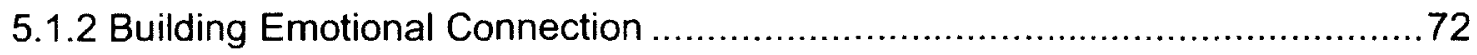

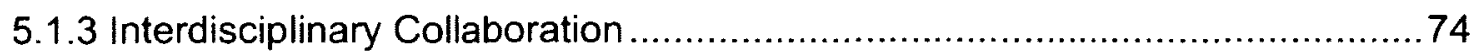

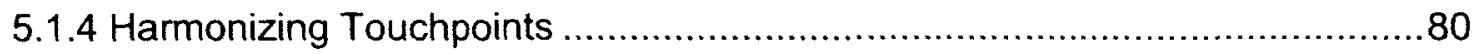

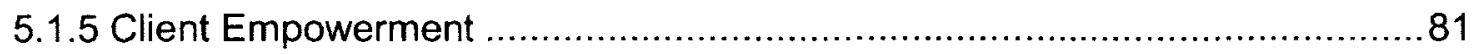

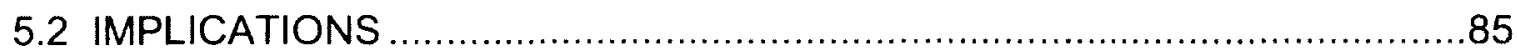

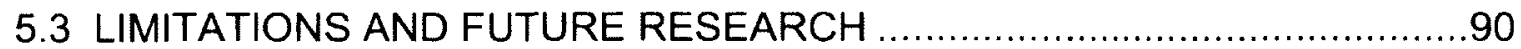

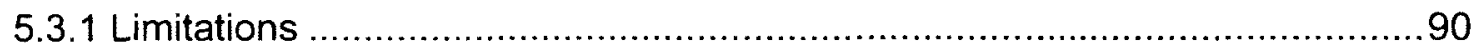

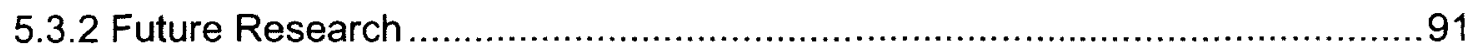

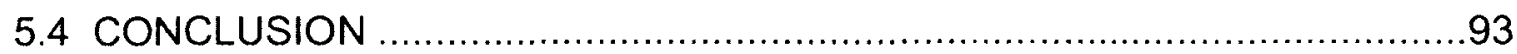

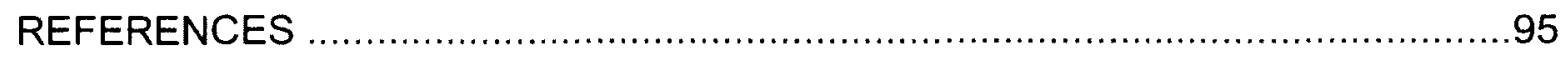




\section{LIST OF TABLES}

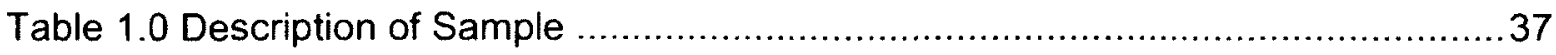

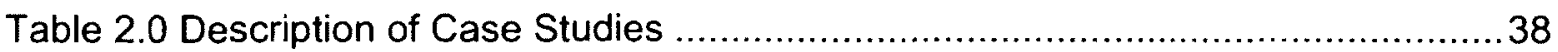

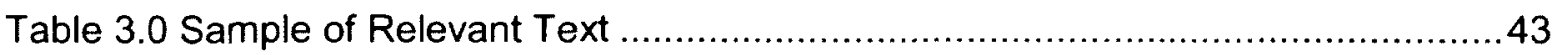

Table 4.0 Sample of Repeating Ideas .................................................... 44 


\section{LIST OF VIGNETTES}

Vignette 1.0 Philosophy Behind the Holistic Perspective ......................................53

Vignette 2.0 Building an Emotional Connection to Foster Rich Experience .................57

Vignette 3.0 Human Resource Allocation for Rich Experience Design ........................60

Vignette 4.0 Harmonizing Touchpoints for Rich Experience Design ......................64

Vignette 5.0 Useful Tools to Empower Clients .................................................69 


\section{LIST OF FIGURES}

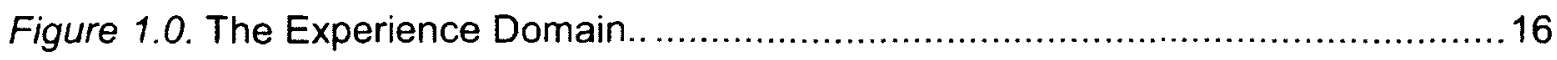

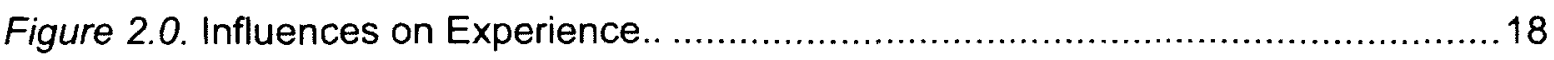

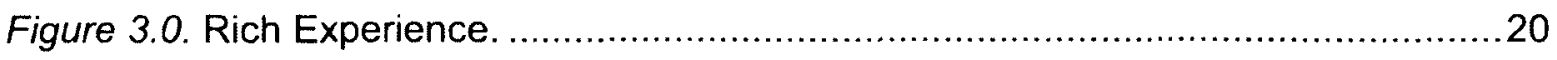

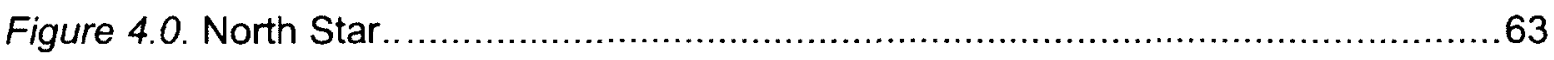

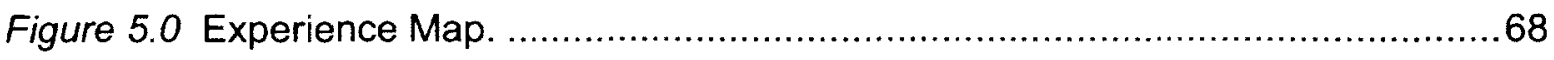

Figure 6.0. Building an Emotional Connection............................................ 74

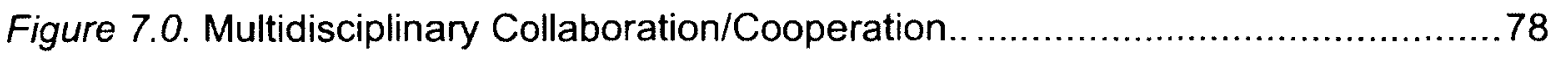

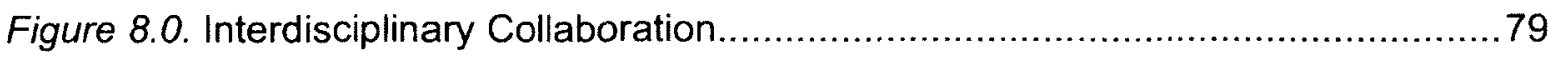

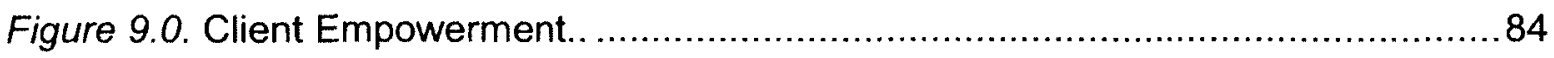

Figure 10.0. Rich Experience Design Diffusion Approach................................ 87 


APPENDIX A




\section{GLOSSARY OF TERMS}

User-customer Journey: a user-customer journey denotes all of the activities that a customer comes across while engaged with a service and extends to include all userproduct and user-service interactions post-purchase.

Product Ecosystem: A product ecosystem is a dynamic unit consisting of "interdependent products and users, functioning together with its surrounding ambience, as well as their interactive relations and business processes ( $\mathrm{Zhou}, \mathrm{Xu}$, and Jiao 2011 , p.45)."

Rich Experience: Rich experiences are generated when a person forms an emotional connection to an interaction with an element of their environment. They are formed as the result of a series of smaller, satisfying experiences throughout the user-customer journey.

Touchpoints: Touchpoints occur whenever a customer or user come into contact with a part of an organization, whether it be a product, interface, customer service call, in-store interaction, or point of purchase display. They occur across several channels and at multiple points in time. 


\section{CHAPTER ONE}

\section{INTRODUCTION}

"A lot of people in our industry haven't had very diverse experiences. So they don't have enough dots to connect, and they end up with very linear solutions without a broad perspective on the problem. The broader one's understanding of the human experience, the better design we will have."

- Steve Jobs

Today's competitive market of goods and services has led companies and designers to seek out continual means of creating indelible impressions for their users and customers through the products and services they offer. Those companies effective in this can evoke emotions of excitement, anticipation, and joy, and are even synonymous with falling in love. The successes are easily identified, they are the companies that have gained international renown and are used time and again as examples of great marketing, design, and service. Oftentimes, it can be difficult to articulate why these experiences, like those had with Apple, IKEA, Porter Airlines, or Nike, are so memorable, they simply possess a "wow" factor that compels brand loyalty and repeat visits.

Just as the winners are easy to spot, so are the failures; they may be beautiful or embody a good idea, yet remain unsatisfying. Often where these companies fail is in disregarding the complex system of interconnected people, products, services, and contexts attached to their own products and services (Brown 2009), leading to negative user experiences along the way. For example, consider trying to find information on a 
company website, then calling customer service and enduring long wait times, before being redirected, repeating oneself to multiple different call staff. It is the sum total of all these individual experiences which either leave a person feeling enthusiastic and satisfied, or in this case, disappointed and frustrated with a company or brand.

Thus, how do highly regarded companies such as Apple and Starbucks create their coveted products and outstanding services? This is a mystery that intrigues today's corporate world, where the competition to stand out amongst competitors is steep. Beyond the great execution of a great idea, one element helping these companies is that they provide their customers and users with something more valuable than the functional fulfillment of needs (Brown 2009). They allow users to connect to the products and services on an emotional level (Pullman and Gross 2004) and to create meaning (Nelson and Stolterman 2003).

Separating the breakthrough products and services from the ones that fall flat, is the total experience they offer their users and customers - from the smallest of details in the product packaging to the design of the product itself, all the way to the customer service interactions and the company brand. This research is based on understanding the factors that result in this ability to move seamlessly between the macro and the micro while designing every component of a system to foster rich experiences at each stage.

\subsection{STATEMENT OF THE PROBLEM}

In such a competitive environment, with growing complexity and increasing user and customer expectations, companies are searching for innovative ways of differentiating themselves. Consequently, many of them have begun to recognize the impact of great experience design and are imploring design-consulting firms to help facilitate this shift. In turn, these firms are allocating more time and resources to creating 
rich experience, as well as to understanding the intricacy of the social systems and product ecologies within which they work (Forlizzi 2008).

However, as demand in this area expands, these consulting firms must develop new design tools and methods leading to memorable, compelling and engaging user and customer experiences, through the products and services they create. In addition, they must adapt their organizational competencies in order to foster design teams capable of embracing and managing the inherent complexities of relational elements impacting the overall experience. Subsequently, designers should look to tackle experience design from a systems perspective.

This notion, coupled with the intrinsic messiness of our lives today, presents designers and companies with "wicked" problems - defined as ill-structured, constantly evolving problems, involving multiple stakeholders of differing perspectives (Conklin 2006). Traditional design and research methods are proving to be insufficient for addressing these sizeable, interconnected networks of people, things, and environments (Pourdehnad, Wexler, and Wilson 2011). As such, critical components of systems are overlooked, resulting in products and services designed in isolation, often leading to market failure (Conklin 2006).

While experience design as a larger field of research has most often been tackled from the angles of human-computer interaction $(\mathrm{HCl})$ (Garrett 2003), marketing, and service design (Pullman and Gross 2004; Schmitt 1999; Stuart 2006; Zomerdijk and Voss 2010 ) it is a relatively new area of investigation for product designers and has had little practical application in industry for their referral. Although these explorations from other fields are important for expanding our understanding of experience and certainly for contributing to the design for experience from a scaled perspective, further inquiry is needed. 
Therefore, the research of this thesis is situated within the context of rich experience design from a systems perspective, and focuses on new methodologies for consulting firms and designers involved in the delivery of rich user experience for corporate organizations with a mainly product based offering.

\subsection{BACKGROUND AND NEED}

Epistemologically, an experience is defined as being yielded when a human comes into contact with any element of nature, whether that is another human, product, or environment (Dewey 2005). For the purposes of designing for experience a distinction must be made between basic human experience and rich experience. Rich experiences are seen as memorable and compelling, and help to increase brand loyalty and satisfaction (Shedroff 2009). They are also generated when a person forms an emotional connection to an interaction with an element of their environment (Pullman and Gross 2004). Authorities on experience span from the theoretical investigations of researchers such as John Dewey (2005) and Mihaly and Isabella Csikszentmihalyi (1988) who studied experiences and how they come to be, to the investigation of their practical application in design by researchers such as Liz Sanders (2001) and Nathan Shedroff (2009), who outlined the specific, reproducible, and controllable elements leading to the creation of memorable and compelling experiences. These authors have described the influencing factors on experience, such as context and culture as well as the components that are paramount in fostering rich experience, such as emotion and meaning creation. These elements are profoundly interconnected and help to form the foundation of designing for rich experience from a systems perspective.

In tackling systems and experience, marketing has established a customeroriented business philosophy referred to as the marketing concept. This philosophy 
centers on coordinating all marketing activities and business efforts across an organization, whether they have to do with planning, product development, pricing, operations, or customer service, etc., (Sommers and Barnes 2004) to create customer satisfaction. The marketing concept has helped to direct the marketing field toward fostering long-term customer relationships and away from its earlier focus on products. The concept determines that every employee or department a customer interacts with is ultimately responsible for the overall customer satisfaction (Sommers and Barnes 2004), thus recognizing that all people, places, events, and things surrounding the organization are intertwined as part of a system. While this philosophy has permeated throughout the marketing domain, it nonetheless lacks critical integration of the design field.

Significant research has been completed on how brands can create experiences (Pullman and Gross 2004; Schmitt 1999). As such, the subject of rich experience cannot be broached without bringing up the value of brand. As the piracy of ideas becomes increasingly common and BRIC (Brazil, Russia, India and China) countries fail to recognize copyright laws, personal interactions with brands are becoming more important than ever (O'Reilly 2012). At present business models based on copyright are becoming obsolete, and companies must learn to distinguish themselves in new ways or feel the consequences of an Internet dominated era where design and technology are easily copied (O'Reilly 2012). Thus, developing long-term brand-customer relationships may be effective in challenging some of these issues (Sommers and Barnes 2004; Veloutsou and Moutinho 2009). Brand relationships are strongly impacted by brand reputation and more importantly by the social influence of brand communities or brand tribes (Veloutsou and Moutinho 2009). Therefore, brands will need to create intense customer relationships built on experiences, as relationships; culture; and experience are unique to the individual and as such, cannot be duplicated (Arnould and Price 1993; Pine and Gilmore 1999; Walls et al. 2011). 
This, along with the assumption that in today's world, especially in the affluent Western societies, people's basic needs have been fulfilled, has led to the emergence of the experience economy, where companies compete on the basis of experiences (Pine and Gilmore 2011). Consequently, user and customer expectations of the products and services they use have grown exponentially. It is no longer sufficient to simply have products and services that are functional - they must now be meaningful and emotional in order to have value. As such, many companies have shifted to focusing on the delivery of experiences. This has brought about the advent of so-called experience brands, like Disney and Virgin America (Brown 2009), as well as experiential marketing (Schmitt 1999). However, this type of experience design is focused primarily in the world of services and little investigation has been completed on the role of products within a larger network of services, people, and environments.

As producing exceptional experiences through services has been thoroughly investigated, the secrets behind thriving experience brands in the hospitality and tourism industries, as well as specific "how-tos" for service and marketing professionals have been uncovered. Experts such as Tim Brown (2009), Pine and Gilmore (2011), Zomerdijk and Voss (2010), and Pullman and Gross (2004) all provide the tips and tricks necessary for any company looking to create remarkable customer experiences through their services. Concepts like engaging front line employees (Brown 2009; Zomerdijk and Voss 2010), sensory design (Pine and Gilmore 1998; Shedroff 2009; Zomerdijk and Voss 2010), and minimizing negative cues (Pine and Gilmore 1998) have all been proven as successful techniques. However, this study takes a new perspective on the concept of delivering exceptional experiences by tying the customer's journey with that of the user's, as more often than not, the user is the customer and vice-versa. Traditionally, in the realm of product design, the definition of a user was restricted to the use of a product or interface, while the definition of a customer was limited to purchasing 
activities, directly involved with the service sector of a company. This may largely be due to the fact that, until recently, the design and marketing of products were separate from the product exchanges of the people who buy them, therefore focusing mainly on userproduct interactions and less on customer service interactions. Hence, by broadening this segmented perception, not only can the elements that these groups encounter individually be understood, but also their experiences across all touchpoints within a company can be assessed.

Subsequently, by expanding the perspectives of users and customers, it is possible to begin appreciating a company's products and services within an entire system. Thus, for the remainder of this thesis, the terms "user" and "customer" will be used interchangeably. In discussing complex systems, design thinking, in its ability to utilize varying perspectives and problem solving techniques, has recently been seen as effective in attempting to tackle the "wicked" problems that emerge from the web of interconnected people, things and contexts (Brown 2009; Pourdehnad, Wexler, and Wilson 2011). However, design thinking alone is insufficient, as it breaks down systems into their constituent parts, losing the overall essence that defined the system to begin with (Nelson and Stolterman 2003). Therefore, in order to design for a world with so much complexity, designers must shift their point of view from the isolated to the connected, wherein services and products are linked in a comprehensive system. Accepting this paradigm shift will require the design industry to develop new business models that will enable professionals to understand and design for today's complexity and the impending business challenges. More specifically, this study looked at product ecosystems. It takes a broader approach, moving outside the singular service, product, packaging, or interface, to understanding the connections, relationships, and patterns between all the elements of an ecosystem and how they can come together to create rich user experiences. 


\subsection{PURPOSE OF STUDY AND METHOD}

Through an investigation of experience-centric design consulting firms, this study set out to uncover the methodologies and organizational behaviours that should be embraced when designing for rich user experience.

As discussed, designing for experience has had a broad application in the realm of services (Pine and Gilmore 1999; Schmitt 1999; Stuart 2006; Zomerdijk and Voss 2010), but very limited application in the area of product design. However, moving beyond the concept of a single service or a single product to understanding the total customer experience governed by the interactions within a system of interconnected products and services, presents a new design space and opportunity.

A few consulting firms have begun to embrace customer experience design through a systems lens - notably, firms such as IDEO, Ziba Design, Adaptive Path and Design Continuum. Still, their processes in this area are not well documented and warrant further examination. The common thread connecting these firms, regardless of whether they are product or service oriented, is that they have approached the design of their client's offerings consistently, harmonizing all touchpoints throughout the customer journey. The concept of rich experience design, utilizing a system's approach, has been loosely referred to in trade literature (Merholz et al. 2008; Brunner and Emery 2009). These authors provide several valuable tools that companies wishing to create products of desire can implement. While the suggestions they provide through anecdotal evidence is of significance to the field, an empirical study of the components that are critical to creating successful user experiences has yet to be executed.

In order to understand this, multiple case studies of seven "experience-centric" (Zomerdijk and Voss, 2010) design consulting firms were carried out, investigating firms which are focused on fostering rich user experiences through the products and services 
they create. The primary source of data collection involved in-depth interviews with experts from each firm who were heavily involved in the design and development of products, interfaces, and service design through a systems lens. Subsequently, a document analysis of each case firm was carried out and served to crosscheck and if possible, corroborate and support the evidence found through the interviews. Data analysis primarily involved coding and categorizing ideas into major themes. The research questions, as well as the literature review, served as the theoretical framework through which to carry out the investigation.

After identifying consulting firms which at present, are successfully designing for rich user experiences by approaching their offering as a system, this study hoped to uncover the fundamental components of the services and products responsible for great user experiences, as well as how these lessons could be applied to other businesses and designers. This research aimed to develop a set of design patterns that would form the foundation for future research in building specific principles or guidelines to aid designers and company visionaries in designing meaningful experiences through their products and services. Furthermore, as any consulting firm wishing to adopt an experience culture inevitably involves some level of organizational change, this study aimed to discover the central organizational behaviours of existing firms which place user experience at the core of their offering. In addition, the research looked to delve more deeply into the specific methods and processes they used in achieving this and executing the design for rich experience.

\subsection{RESEARCH QUESTIONS}

Through an empirical investigation of design consultancy firms at the forefront of experience-centric product and service design from a system's perspective, as well as a 
review of relevant trade and academic literature, this research aimed to answer the following questions:

R1. What are the components or elements that lead to rich user experience?

R2. What are the organizational behaviours and perspectives that must be adopted by design consulting firms shifting focus from the delivery of products and services to fostering rich user and customer experiences?

R3. What are the most effective design processes and practices being utilized by experience-centric design consulting firms that ultimately give rise to rich user experiences?

\subsection{SIGNIFICANCE TO THE FIELD}

This research has significant implications for the design field and may also be beneficial to professionals involved in operations, helping them to drive organizational and marketing focus within a business. Furthermore, this study will provide valuable insight into the elements that should be considered when developing innovative, experience-centric products and services. As an aside, the insights uncovered will bring light to a vague area of design and as a result may also aid design educators in articulating the abstract concepts of experience, as well as assist them in developing coursework centered around experience design as the field moves towards this new horizon. Moreover, the design patterns identified will provide information to managers on the qualities to look for in designers during the hiring process and consequently, the areas designers in the field should focus on when developing their professional skills and experience. 


\section{CHAPTER TWO \\ LITERATURE REVIEW}

Successful products like the iPod and services like Starbucks have caused an industry wide lust for the elusive "wow" factor - the element that can elevate a product or service and allow it to outperform its competitors. This has led to a shift in the design discipline away from its traditional focus on the "artifact" and towards a larger system of experiences. In fact, Sanders described a new design space where "designers [would] transform from being designers of "stuff" to being the builders of scaffolds for experience" (Sanders 2002, 3).

Increasingly, companies are seeking novel means in differentiating themselves as the products and services they offer are easily reproduced (Pine and Gilmore 1998; Pullman and Gross 2004; Walls et al. 2011). As such, the opportunity lies in designing for rich user experience, an attribute much harder to replicate if done well (Garrett 2003; O'Reilly 2012). While extant literature on experience design has been written from the perspectives of services research, marketing, $\mathrm{HCl}$, operational management, and hospitality (Pine and Gilmore 1998; Pullman and Gross 2004; Sheng and Chen 2011; Stuart 2006; Zomerdijk and Voss 2010; Walls et al. 2011), there are relatively few studies completed from a product design perspective. However, central to the concept of experience is that they exist as a result of human interactions with products, not only with services.

Furthermore, fundamental to the industry of product and services development, yet often neglected, is the notion that nothing exists in isolation (Nelson and Stolterman 2003), and as such everything is a part of a larger system of services and products 
offered within an organization. Every component of that system is interrelated and in order to evoke a wholly rich user experience and achieve the "wow" factor, they must work together harmoniously, evoking satisfying user experiences at each stage within the product and service lifecycle.

In this chapter, an in-depth literature review on the theoretical underpinnings of human experience is presented, as well as a comprehensive overview of its applications in the service and $\mathrm{HCl}$ sectors to date. The latter helps to demonstrate the advantage of user experience design and conveys why further investigation into experience from a product and systems perspective is needed. In essence, experience has been investigated from various disciplines and each has discovered similar triggers leading to positive user experience. This review will examine the principles utilized by these respective disciplines, and which, if any, can be carried forward by experience-centric design consulting firms. Secondly, rich or extraordinary experience is explored, uncovering the fundamental components that should be critically considered by designers wishing to foster intrinsically enjoyable encounters between users and companies. Finally, the notion of systems thinking and product ecosystems is presented and examines how designers can approach rich user experience design from a systems perspective, helping to set the context for the empirical study.

\subsection{EXPERIENCE DESIGN}

\subsubsection{BACKGROUND}

In 1999, "The Experience Economy" written by Pine and Gilmore forecasted a significant transformation of the consumer goods and services industry, stating that experiences would provide companies an increased competitive position as an added value in the saturated market of goods and services. In particular, they described an 
economic progression from commodities to goods; goods to services; and ultimately from delivering services to staging experiences. While the concept of experience had previously been categorized under the larger umbrella of services, Pine and Gilmore (1999) made a clear division between the two, arguing that staging experiences presented a distinct economic offering. Explicitly, where services were understood as intangible and customized to the customer; experiences were memorable and personal to the individual. Nevertheless, both were described as profoundly intertwined: The service is the stage upon which the experience occurs.

The latter relationship launched a revolution prevalent in the service sector and directed marketing teams to explore new and innovative ways of building stronger customer relationships (Pullman and Gross 2004; Schmitt 1999; Walls et al. 2011). This led to the birth of Experience Design, more accurately represented as designing for experience, as experiences are subjective events that cannot be designed, only stimulated (Schmitt 1999). Numerous experts have established service design principles effective in fostering positive customer experiences (Pine and Gilmore 1998; Zomerdijk and Voss 2010; 2011; Pullman and Gross 2004; Stuart 2006). While each author has an individual interpretation of these principles, they revolve around similar themes: Storytelling (Shedroff 2009), sensory design (Zomerdijk and Voss 2010), harmonizing touch-points (Pine and Gilmore 2011), designing for cues (Pine and Gilmore 2011; Zomerdijk and Voss 2010), engaging customers through front-line employees (Brown 2009; Zomerdijk and Voss 2011; Pine and Gilmore 2011), and minimizing negative effect (Pine and Gilmore 2011; Pullman and Gross 2004). Select principles are revisited in more depth further on in this chapter. Similarly of note are reputable organizations from the hospitality and tourism industries such as Disney or W Hotels which have mastered the art of designing for experience (Walls et al. 2011). Studying these types of companies can provide significant insights, as unlike purely product or service oriented 
organizations, hotels, amusement parks, and museums base their survival on their capacity to create positive experiences for their guests (Pine and Gilmore 2011; Sheng and Chen 2011; Walls et al. 2011).

More recently, in the field of $\mathrm{HCl}$, User Experience (UX) design involves the creation of a computer or web application's user interface (UI), information architecture and visual appearance. In essence, UX looks into the dynamic between user-technology interactions and focuses on their experiential qualities (Hassenzahl, Diefenbach, and Goritz. 2010). In this sense, the elements forming these interactions are designed to be highly usable and realize user expectations (Garrett 2003). As such, industry standards, such as Jakob Neilson's 10 Usability Heuristics (Neilson 2005), have been outlined for best practices and are carefully followed by industry professionals.

The level of interest arising from these respective disciplines in this area is not surprising, as the impact of good experience design is unmistakable. However, only of late has experience design gained more widespread attention from designers traditionally involved in the development of products (Brown 2009; Merholz et al. 2008; Shedroff 2009).

Regardless of the medium, whether a service, product, media, print, theatre, or interactive experience, if designers are to develop truly compelling experiences for customers and users across a company, the solutions require a deep understanding of experience, as well as its' causes or triggers. Subsequently, these multidisciplinary perspectives on best practices and the science of user experience are valuable in identifying the influencing factors leading to good or bad experiences, as well as the elements that can be manipulated in order to foster positive interactions with a company or brand. From the literature three main categories could be drawn out as crucial for designing rich experiences: Context, Meaning, and Emotion. While these three elements are intertwined they are discussed individually in the subsequent sections. This research 
helps to establish the framework from which to develop new solutions for design consultants dealing with complex systems, involved in rich experience design.

\subsection{BASIC EXPERIENCE}

In order to design for experience, particularly rich experience, we first examine its rudimentary definitions and epistemological foundations. John Dewey (2005), renowned philosopher and pioneer in demystifying human experience and expression from an aesthetic perspective made significant contributions to the field in his book "Art as Experience". At its' most basic, he described an experience as being yielded when a person interacts with any element of the world in which they live. Thus, every interaction is an experience, whether it is human-to-product, human-to-environment, or human-tohuman. Similarly, Zomerdijk and Voss (2010) investigated the customer experience from a services perspective and referred to it as occurring "when a customer has any sensation or acquires knowledge from some level of interaction with the elements of a context created by a service provider" (p.67).

What is more, Mihaly and Isabella Csikszentmihalyi (1988) explain that the activity and the experience are independent of each other and "only the interaction between the two can lead to answers as to why an activity fosters an enjoyable experience" (p.85). Thus, the key to eliciting satisfying experiences is in making each interaction pleasurable (Popovic 2002).

Dewey's (2005) interest lay in what he referred to as "real experience", not a passive event, but one that can be recalled as either good or bad, referenced by saying "that was an experience". According to his work, this type of "real experience" is not infinite, but rather has a beginning and an end. Therefore, in order for an experience to transpire, the event in question must first reach fulfillment - defined by what went before 
and what will come after (Dewey 2005). He stressed that experiences have more depth and intensity when the person has sufficient past experiences to fully understand their present experiences. Sanders (2001) explained experiencing as the point where memories and dreams meet (depicted in Figure 1.0). This indicates that our past experiences play a significant role on how we interpret our present world, while our future goals drive and shape current experiences. Consequently, a sweet spot exists for designers focused on understanding a user's past experience together with their dreams, allowing them to uncover a user's motivations and latent needs in order to best mould rich experience (Sanders 2001).

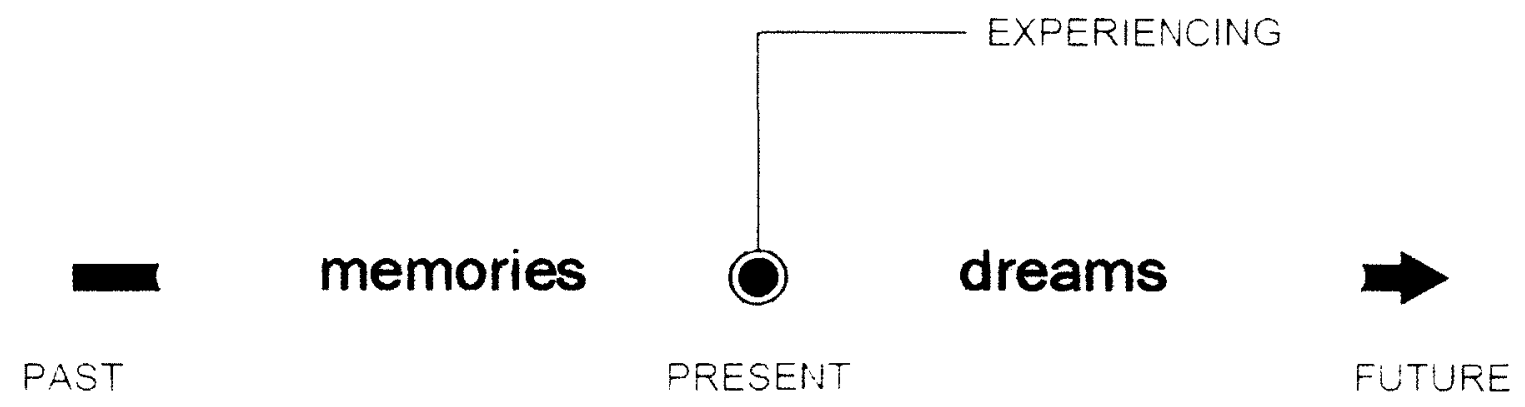

Figure 1.0. The experience domain. This figure depicts the point at which experiences occur through time. Source; Adapted from Sander's (2001).

As the dialogue on experience progresses, a brief inquiry into the types of experience under investigation is necessitated. An individual will have various experiences every day, some of more significance than others, hence considering them all would vastly limit the feasibility of this study. Therefore, a distinction must be made between what Egall et al. (1997) referred to as a common "consumption" experience and a "consumer" experience. A consumption experience is equal to the ordinary experience of everyday life and may involve activities like picking up your child from school or a dinner party with friends (Egall et al. 1997). However, this is outside the realm of the marketplace and therefore of less concern to those individuals designing interactions between people and products, and services. In comparison, consumer experience is 
constrained to product and service exchanges (Egall et al. 1997) and is a central concern of this study.

Regardless of whether the experience is of a canoe trip or an online banking session, each has an identifiable structure and a pattern (Dewey 2005). Namely, a singular experience is made up of a countless number of smaller experiences culminating when the activity comes to an end (Forlizzi and Ford 2000). In addition, an experience as a whole has unity, which defines it. Dewey (2005) postulated that emotion helps provide this unity in and through the varied parts of an experience. Therefore, binding these constituent experiences into a full and complete experience requires a common quality that pervades throughout. This quality is one that Dewey stipulated must be "esthetic". The latter demarks Dewey's (2005) great insight and central contribution: He defined experiences as having an "esthetic" quality if there is a desire by the person involved to have accomplished a process - described as an urge to fulfillment and a feeling and interest towards the things that happen along the way, either hindering or helping the experience.

As previously mentioned, experiences are highly subjective and as Sanders (2001) noted, they are innately personal. Thus, activities, specifically human-to-product interactions, evoke different meanings for different people. For this reason, designers cannot design an experience. They can only design elements that people can interact with to stage desired experiences (Forlizzi and Ford 2000; Sanders 2001; Pine and Gilmore 2011). Explicitly, "within management's domain, the service designer can design for experience and operations manager can facilitate an environment for experience by manipulating key elements" (Pullman and Gross 2004, 552).

Nonetheless, there are factors influencing how a person perceives an experience, as either pleasurable or uncomfortable. Forlizzi and Ford (2000) described 
these factors as including elements of emotion and context. To understand this better, consider the following diagram (Figure 2) of influences on experience.

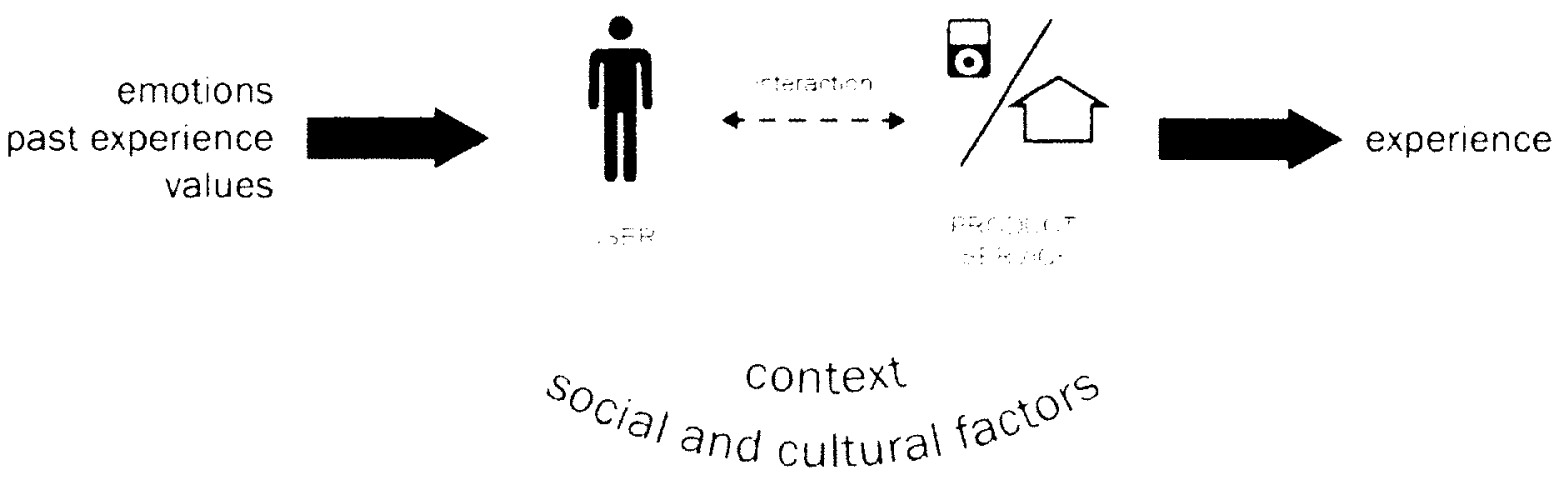

Figure 2.0. Influences on experience. This figure depicts the influencing factors on a user's experience through user-product and user-service interactions. Source; Adapted from Forlizzi and Ford (2000).

Considering these elements, along with meaning creation, is crucial for designers and managers, as even sitting in a coffee shop, driving a car, or watching a movie, denotes an experience - not simply a person carrying out a function. Thus, each of these activities and subsequent interactions is an opportunity for designers to design the root components of rich user experiences.

\subsection{RICH EXPERIENCE}

For the purposes of designing for experience a distinction must be made between basic human experience and rich experience. The emphasis placed on rich experience results from the notion that designers and companies ultimately seek to deliver memorable and compelling experiences to their users through the products, services, and systems they design. 
Having understood the foundations of experience, several authors have progressed to explore the causes behind peak, optimal, or transformative experiences (Abraham 1986; Arnould and Price 1993; Csikszentmihalyi and Csikszentmihalyi 1988; Thorne 1963; Walls et al. 2011). In particular, Mihaly and Isabella Csikszentmihalyi (1988) assert that in order for optimal experience to exist an individual must enter a state of consciousness referred to as "flow". They maintain that flow can only be reached when the activity a person is engaging in has a clear set of goals and provides immediate feedback as to how well they are advancing through the task. Subsequently, when an individual's skills meet the goals with the right equilibrium of challenges, "flow" develops. Csikszentmihalyi and Csikszentmihalyi (1988) stress that this optimal state of consciousness and therefore optimal experience, can occur during any activity, regardless of whether it is leisure or work oriented. In essence, providing that the abovementioned elements are present, flow can ensue. The characteristics defining an optimal experience derived from this state of flow include deep concentration and immersion, leading to a distorted perception of time, suggestive of the idiom "time flies when you're having fun".

Csikszentmihalyi and Csikszentmihalyi (1988) go on to state that the "activities and experiences that are most enjoyable will have a greater chance of being remembered and of being built into the memory-storage of the culture" (p.34). Therefore, it could be said that the concept of flow is akin to natural selection when it comes to choosing the activities in our lives that are worth pursuing and repeating. Subsequently, if those involved in the design and delivery of user-product or user-service interactions are able to create a state of flow they will, among other accomplishments, engender customer loyalty and repeat purchases as well as strong user satisfaction.

Rich experience is a critical ingredient to creating a successful product or service. Nevertheless, the fact that a product is part of a broader network of products 
and services offered within a business, intended or not, is often neglected. A rich user experience can only be achieved when every component of that network works together, evoking satisfying experiences at each stage along the user-customer journey (Forlizzi, and Ford 2000). Moreover, rich experiences are only generated when a person forms an emotional connection to an interaction with an element of their environment (Pullman and Gross 2004). Following Dewey's understanding of experience, it could be expanded to say that if all experiences have a quality unifying its constituent parts - then if that quality was emotionally rich, we would have rich experience. Therefore, rich experiences are created as the result of a series of smaller satisfying experiences, unified within a product's ecosystem (Forlizzi 1997). Figure 3.0 depicts how a rich user experience can be fostered.

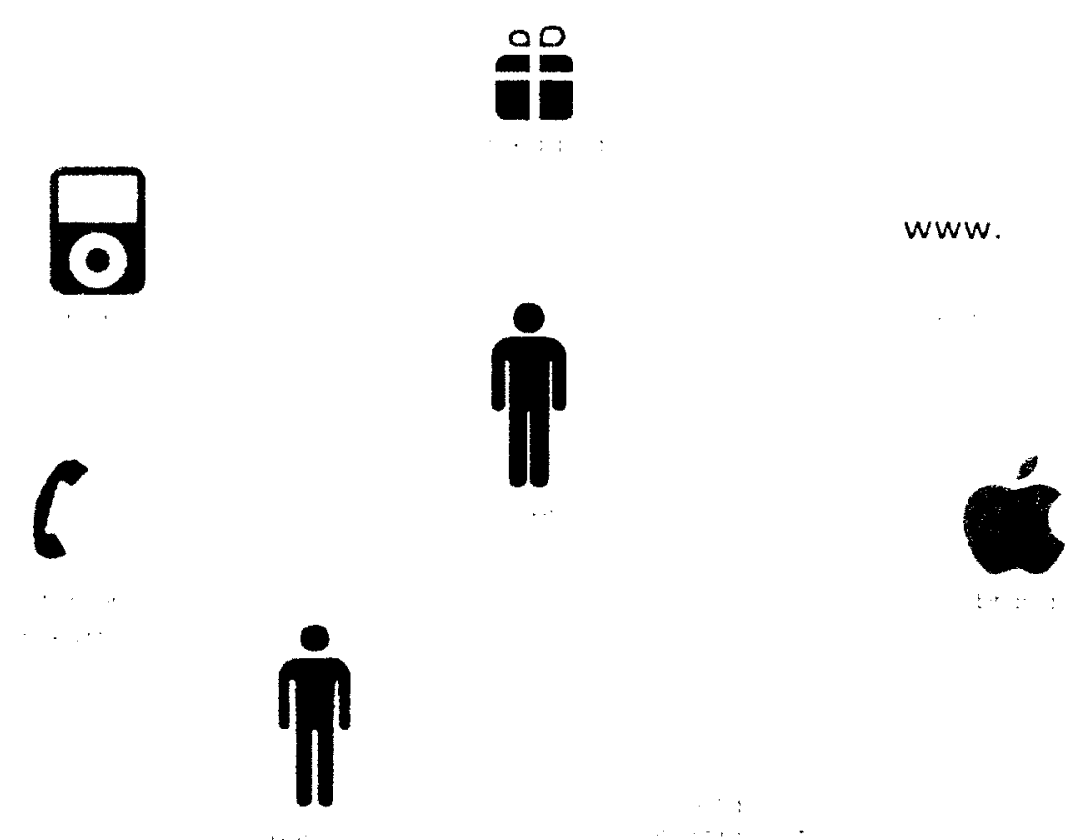

Figure 3.0. Rich experience. This figure illustrates how a rich experience can be fostered through a collection of satisfying, emotionally charged interactions between the user and each touchpoint. 


\subsubsection{CONTEXT}

Perhaps the single most influencing factor on experience is that of context. Irrespective of the discipline in which experience design has been studied, the concept of context has most often been identified as its primary concern (Merholz et al. 2008; Zomerdijk and Voss 2010; Pine and Gilmore 2011; Pullman and Gross 2004). Explicitly, experiences are shaped by the context in which they are lived, therefore if the context changes so shall the experience (Forlizzi and Ford 2000). What is more, context is heavily affected by a person's past experiences and culture, including societal norms (Merholz et al. 2008; Khalid and Helander 2006).

The notion of context can be understood from two separate standpoints. The first, a perspective from the services sector, explains context as consisting of all the physical and relational components in an environment (Bitner 1992; Pine and Gilmore 1999; Pullman and Gross 2004; Zomerdijk and Voss 2010; Walls et al. 2011) and these elements can be designed to evoke meaning and emotions among users. The second is more commonly referred to in product and UX design and that is the context of use that the product will be used in, whether that is a car, a kitchen, or an office. The key difference between these elements is that the former is controllable and designable, whereas the latter cannot be controlled and is often difficult to predict. Nonetheless, the context of use it is just as critical to product experience as physical and relational environmental aspects are to service experience. However, both types of context must be well understood when considering entire systems of products and services.

Service Context. The successful management of a product's environment before and after purchase is paramount to the creation of rich experience. In particular, the manipulation of a service's physical context has been established as a successful method in attempting to create an emotional connection between a company and a customer (Pullman and Gross 2004), as the components of a physical environment are 
controllable and therefore designable. One way to manipulate the environment is to design in a manner that engages the user's five senses. Sensory design which has been included in design principles outlined by Pine and Gilmore (2011), Shedroff (2009), and Zomerdijk and Voss (2010) involves the incorporation of sight, sound, smell, touch, and taste into a product or services environment. It is a technique that has proven to be particularly useful in the design of retail atmospherics (Bitner 1992). For example, IKEA stores encourage customers to touch, feel, and try the furniture, helping them to envision these products in their home, ultimately leading to more purchases. In stark contrast, are the home and housewares stores, such as Structube, where customers are discouraged from trying out the furniture. Although it is not explicitly against the rules the atmosphere and even the staff do not engender a sense of comfort and connectivity. This is just one of the reasons why IKEA has become a household name around the world. IKEA is not the only company to use these techniques; Starbucks also incorporates sensory design by playing coffee house music in their stores, using ambient lighting, and taking care to brew a fresh pot of coffee every half-hour for its delectable aroma. All of these aspects are carefully designed to coordinate with the brand and all other customer touchpoints in the product ecosystem. By engaging a customer's senses you enhance their experience: "The more effectively an experience engages the senses, the more memorable it will be" (Zomerdijk and Voss 2010, 69).

However, beyond designing the physical/tangible elements of an environment, it is also necessary to manage the relational elements of the environment, through the social interactions between customers and employees, as well as the interactions between customers themselves (Pullman and Gross 2004; Walls et al. 2011). Likewise, Abraham (1986) stated that these interpersonal interactions are an important trigger leading to intrinsically enjoyable experiences for the customer. Therefore, an effective means of staging experiences is by managing employees to engage with customers in a 
targeted and socially appropriate way (Walls et al. 2011). For instance, in trying to foster positive interactions between customers and employees Apple created the "Genius Bar" at all their retail stores, where customers can ask an Apple Genius about any technical problem, adding a sense of playfulness, while engendering trust and making the experience memorable. Similarly, Disney trained employees to always look like they were having fun, while MacDonald's trained them to be enthusiastic with a good sense of humour (Arnould and Price 1993). Pullman and Gross (2004) found that the relational elements of a service environment strongly affected guest emotions, more so than the physical context.

Context of Use. Additionally, the study of a product's context of use can deliver vital insights into a user's goals and motivations. While a designer may have no control over the context of use, it must still be considered and extensively researched in order to achieve a holistic design (Forlizzi and Ford 2000). Both product design and UX design, are highly context dependent (Khalid and Helander 2006; Merholz et al. 2008); while in some cases the context of use may be simple to predict, oftentimes, especially when dealing with entire user-customer journeys, as design for rich experience requires, it is not as easily captured.

\subsubsection{EMOTION}

The second element impacting the design of experience is emotion, as our emotions govern how we feel, think, and behave in every situation. In fact, emotion and experience are so deeply intertwined that one cannot occur without the other (McCarthy and Wright 2004). Moreover, as Dewey (2005) noted, a prominent emotional quality resulting from a single interaction can be amply dominant to mark the experience as a whole. In establishing the impact emotion has on a customer experience, Pullman and Gross (2004), completed an empirical study of service elements created to elicit 
compelling experiences at a VIP hospitality tent for a world-renown circus. Most notably, their study revealed that the customer's emotional behaviour significantly impact their loyalty behaviours.

As such, the foundation of emotional design lies in trying to maximize positive emotional reactions and minimize negative reactions to a product or service (Khalid and Helander 2006), thereby eliciting pleasurable interactions. In fact, two of Pine and Gilmore's (1998) five experience design principles demonstrate the latter. They stated that by eliminating negative cues and harmonizing impressions with positive cues, indelible impressions that affirm the nature of the experience to the customer are created. The difference is largely to do with the negative emotions attached to warning signs, clutter, dirt, etc., which trigger avoidance behaviours to leave or ignore.

Conversely, positive emotions trigger approach behaviours - involving the desire to stay, interact, and explore (Zomerdijk and Voss 2010). Hassenzahl, Diefenbach, and Goritz (2010) found similar results during their study of 548 positive user experiences with technological products. In particular, they found that psychological need fulfillment led to strong positive affect among individuals, that resulted in a higher perceived product perception and an overall positive experience. While this study assessed experience resulting from user-technology interactions, they can nonetheless be broadened to predict that positive affect reveals positive experience, regardless of the interaction platform.

Traditionally, it was believed that emotion and cognition were separate, however advances in neuroscience have shown that they are in fact linked: "Emotion and cognition contribute to the control of thought and behavior conjointly and equally" (Khalid and Helander 2006, 198). Don Norman (2004), in his book "Emotional Design", described three levels of human emotional-cognitive processes: Visceral, behavioural, and reflective, which he also extended to depict the levels of emotional design. Explicitly, 
Norman (2004) explained visceral design as appealing to the basic senses and is subject to a person's immediate response to the elements in an environment - a gut reaction. In the field of design, this type of reaction is based primarily on the visual aesthetics of a product. Whereas, behavioural design, largely referred to as a product's usability and performance, is of primary concern for professionals in $\mathrm{HCl}$. Comparatively, reflective design plays on cultural and societal norms and can involve the user's social perception of the design. A product or interface's ability to effectively meet this third level of processing, over the first two, has been found to be particularly relevant in the area of experience design (Khalid and Helander 2006; Shedroff 2009) Similarly, Shedroff (2009) argued that experiences can be fulfilling if they challenge a person's beliefs. In doing so they are required to reflect upon their own sense of self and values, allowing the activity and interaction to either affirm or deny their sense of self. Equally, Arnould and Price (1993) described a consumer's satisfaction as relying on whether or not a product or service met their expectations - where expectations include beliefs, evaluative criteria, attitudes and activity sequences. Therefore, designers focused on designing for rich experience might focus on understanding user and customer expectations in order to meet or exceed them. This means they must have a profound understanding of the user, in order to understand their motivations, behaviours (Merholz et al. 2008) and even their dreams (Sanders 2001). Nonetheless, user interactions leading to strong emotional connection should be designed to address all three levels of emotional processing (Norman 2004).

\subsubsection{MEANING MAKING}

Shedroff (2009) believed that meaning is the most important factor of experience. The previous section demonstrated that experiences are emotional, yet we cannot have emotions without connecting or attaching them to an object, person, or event (Dewey 
2005). Csikszentmihalyi (1981) worked to understand the significance people attach to the objects in their everyday lives, and described meaning making as a communicative act, resulting from the perception of an object as a sign or symbol.

As such, several authors claim that meaning creation and experience is incomplete without recounting it through a story (Forlizzi 1997; Forlizzi and Ford 2000). They explained that an experience only becomes an experience once it is translated into knowledge about the interactions or occurrences (Forlizzi 1997). Storytelling is the most common means of communicating our experiences to others every day. "Stories are the vehicles that we use to condense and remember experiences, and to communicate them in a variety of situations to certain audiences (Forlizzi and Ford 2000,420)." Moreover, we relate to products through storytelling by attaching meaning to products, as such they become part of our own life story. A product can either affirm or challenge our beliefs, giving it the power to alter a story line (Shedroff 2009). Similarly, Nelson and Stolterman understood meaning making as "the creation of which is experienced and the one who experiences. These relationships form a belief system, inclusive of the real, the true and the ideal, that informs actions, reflection and imagination in specific situations" (2003, 80).

Effectively, storytelling is so vital to the user-customer journey that theatre has been used as a model for staging experiences (Stuart 2006; Pine and Gilmore 2011); where employees are actors, the physical environment of the service is the stage, products are the props, service delivery is the script, and the customers are the audience. This metaphor has helped companies to unify the customer journey. The latter can also be strengthened by themeing the experience: "The theme must drive all the design elements and staged events of the experience towards a unified story line that wholly captivates the customer" (Pine and Gilmore 1998, 103). 
In essence, a product is a narrative; a formal representation of values and beliefs of the designers who created it. A product can communicate to a user through its affordances $^{1}$ and aesthetic - it may communicate to them a sense of social status or how it should be used. According to Forlizzi (1997), when a product's narrative interlaces with the user's own past experiences and life story, rich experiences are yielded. This process of storytelling helps users form meaning when interacting with a product. Meaning can foster customer loyalty, affirm the user's own sense of self, create value and is the underpinning of experience (Shedroff 2009). The emotion that a product engenders along with the context, will shape a product's narrative to an individual user.

In the end, meaning creation only exists as a result of interpreting our world through the relationships and interactions occurring within a context. Thus, artifacts, services, interactions, activities only become significant when understood and communicated as part of a broader system.

\subsection{SYSTEMS}

\subsubsection{SYSTEMS THINKING}

Systems thinking is a way to approach meaning making by forging the relationships between elements. It allows us to gain a more comprehensive understanding of our world and the elements within it. According to Nelson and Stolterman (2003), systems thinking is the rational component of design and helps to bridge the gap between two cognitive counterparts: The imaginative, intuitive, and emotional side with the analytic side.

\footnotetext{
Affordances on products provide clues to the user as to they should be operated. For example a doorknob indicates to the user that it can be turned based on subtleties in its form (Norman. 2002).
} 
While analytic thinking is behind much of design reasoning, they caution that it can lead to logical dissection; in other words, where the study of things is in isolation from their intact whole. Therefore, a solely analytic approach results in artifacts, services and process designed within a vacuum. When this happens the emergent quality of those artifacts, services and processes is lost. For example, if while studying a family or community, individuals are separated from the unit, the very essence of what was under investigation is lost, as a brother or sister are only so in relationship to one another. Nelson and Stolterman (2003) refer to this as the overarching quality delineating the system, similar to Dewey's (2005) view of a unifying quality defining an overall experience. Similarly, Pourdehnad, Wexler, and Wilson (2011) noted that "failing to consider the systemic properties as derived from the interaction of the parts leads to sub-optimization of the performance of the whole" (p.3). Thus, it could be interpreted that by analyzing elements or constituent parts of an experience separate from the whole, the overall quality of the total experience is lost. As such, human experiences possess a complex structure and a rich experience must therefore be understood as a complex system (Mutlu 2004).

Today's culture and current design tradition, reason that the more knowledge gained on a particular phenomenon the better. As such, analytic thinking has given birth to a reductionist approach in understanding the world. Reductionist thinking refers to a worldview where everything is reduced and studied through its constituent parts (Pourdehnad, Wexler, and Wilson 2011). Contrastingly, systems thinking focuses on understanding the emergent qualities, patterns and relationships between and across these parts, rather than the specific elements themselves (Nelson and Stolterman 2003). Thus, a systems approach can lead to successful design if the whole remains intact and is understood as a compound of interconnected relationships. 
Take for instance the fable of the blind men and the elephant, each touching different parts in the hopes of understanding what lay before them. In the story, one man touches the trunk and describes the elephant as a snake, while another touches the tail, describing the elephant as a rope, while yet another man feels its tusks and describes a spear. They confer to find only that their individual perspectives were nonsensical when brought together after the fact. Hence, by breaking down or reducing a whole into its constituent parts with no regard for context or systemic influences it becomes impossible to understand the true, complete reality. Whereas a person capable of seeing the whole picture, or in the case of the elephant fable, a man with sight, can understand the elephant for what it truly is.

Another component of complex systems is that of utilizing multiple perspectives to gain a more comprehensive and inclusive view of the whole. By utilizing varying perspectives or frames of reference, for example the perspectives of different stakeholders, can result in different understandings and meaning of the same singular experience (Nelson and Stolterman 2003). This can undoubtedly lead to tensions and paradoxical outcomes. Yet system thinking works to reconcile these differences through the in-depth level of understanding that is inherently missing from the analytic reductionist approach.

Csikszentmihalyi and Csikszentmihalyi (1988) described the main barrier to optimal experience as the tendency towards "rationalization" in Western culture. In particular, rationalization leads to the separation of life functions, the division of labour and creates separate life spheres - for work, school, leisure. This leads to negative or positive connotations of each. This concept is at odds with a human's natural tendency towards understanding their world through relationships (Nelson and Stolterman 2003), as system thinking intrinsically affords: Presenting a challenge for designers, where they must utilize the power of analytic thinking (in its ability to look at one thing in-depth and 
in isolation) but to integrate these individual elements into an overall compositional or systems approach. In doing so, these elements contribute to positive meaningful experiences.

Similarly, systems can be scaled, from the macro to the micro. Bak (1996) explains "I will define systems with large variability as complex. The variability may exist on a wide range of length scales. If we zoom in closer and closer, or look out further and further, we find variability at each level of magnification, with more and more new details appearing. In the universe, there is variability on the greatest scale" (p.5). Moreover, Mutlu (2004) explains that each system has an infinite number of subsystems, each with an infinite number of interactions of varying experiential degrees.

Furthermore, steps in defining and categorizing a system are crucial for designers when considering complex systems, so as not to become overwhelmed by the sheer number of connections. For example, it is possible to have organizational systems, ecosystems, biological systems, etc. These classifications help designers to become more effective in choosing a context and the elements to be investigated and designed (Nelson and Stolterman 2003).

\subsubsection{PRODUCT ECOSYSTEM}

Over the years the design process for a single product has been thoroughly investigated and steadfast principles have emerged, such as considering the context of use, performing design research, and understanding user needs. However, the design of a standalone product is no longer enough to create this "wow" factor (Merholz, et al. 2008). Instead, it is created through the design of a network of harmonized products and services, referred to as the product ecosystem (Forlizzi 2007; Forlizzi 2008; Zhou, Xu, and Jiao 2011). A traditional ecosystem is defined as 
"the complex of a community of organisms and its' environment functioning as an ecological unit" (Merriam Webster Dictionary 2011). Here organisms and their environment are linked together in a life cycle and energy flow, giving and taking from one another. Similarly, a product ecosystem is a dynamic unit consisting of “interdependent products and users, functioning together with its surrounding ambience, as well as their interactive relations and business processes" (Zhou, Xu and Jiao 2011,45$)$. It is important to note the definition of the term product is borrowed from Victor Margolin's definition as the "array of objects, activities services and environments that fill the life-world $(1997,8) . "$ The classic example of a product ecosystem is Apple's iPod. While other MP3 players existed on the market when the iPod was introduced, none of them were conceived of within a system of interdependent products and services. The iPod not only brought simple, beautiful UI and product design, but it was the first music player to incorporate a music management system: iTunes. This allowed users to import music and manage thousands of songs and playlists on a large screen with more capability and functionality than a small screen device could offer. Later, the online music store was added to the system and as the newer versions emerged, the iPod ecosystem became the hub for all media playing, viewing and management (Merholz, et al. 2008).

\subsubsection{PRODUCT ECOSYSTEM AS A DESIGN STRATEGY}

In order to understand this further, the following section discusses the nature of user experience from the perspective of designing an ecosystem.

Forlizzi (2008) developed a product ecology framework that would inform design research methods in order to conduct qualitative research that would lead to the deepest 
insights regarding a user's social context surrounding a product. This framework is informed by social ecology theory. Moreover, Zomerdijk and Voss' (2010) work on experience-centric service organizations discusses the importance of product ecosystem design when creating positive user experiences. After completing 17 case studies with service providers like Disney World and consulting firms such as IDEO, three main principles were found to be especially true in the delivery and design of services eliciting rich experiences: They involve designing the dramatic structure of events, sensory design, and harmonizing touchpoints. Of particular interest, the last proposition states that the design of experience-centric services involves designing customer journeys and harmonizing touchpoints. Zomerdijk and Voss (2010) describe touchpoints as occurring whenever a customer comes into contact with a part of an organization, whether it is a call to customer service, interacting with a product in-store or at home. They are across several channels and at multiple points in time. Although this research stems from a services research perspective, its' essence can be applied to the design of product ecosystems - stressing the importance of a positive experience at each and every stage within the product lifecycle. Likewise, Shedroff (2009) offers the concept of consistency. He states that consistency across all touchpoints leads to a higher level of meaningful connection between the user and product, as well as perceived reliability of a product and brand. However, oftentimes this can be difficult to achieve in large organizations as the separate organizational silos do not communicate with one another, thereby losing the consistency across the touchpoints and resulting in independent units whose lack of coordination causes a mitigated customer experience (Merholz, et al. 2008). Therefore, moving forward, designers must consider the impact of all facets of the product system on the user experience.

In effect, systems thinking is a lens through which to approach our understanding of the world and helps to focus our actions to appreciate the relationships (Nelson and 
Stolterman 2003; Pourdehnad, Wexler, and Wilson 2011). It is not based on specific domain knowledge or scientific disciplines. It is a broader focus on the relationships and emergent patterns between interconnected elements in reality. While, companies are realizing the significance/impact that experiences have on customer loyalty and purchasing behaviours, the specific tools and methodologies elude the design professionals in charge of creating the platforms for these experiences, be they products, services, or interfaces. As such, new principles and methodologies utilizing systems as a lens, must be outlined for consulting firms and more specifically for the designers and researchers directly involved in the design and development of these platforms. Therefore, the following study attempts to elucidate some of the phenomena surrounding rich experience and uncover new methodologies and perspectives through a case study analysis of seven experience-centric design firms. 


\section{CHAPTER THREE}

\section{METHODS}

As previously discussed, the industry landscape of service and product design is evolving towards an experience-oriented culture. As such, designers must adapt to recognize these products and services within an interconnected system if they are to create truly meaningful experiences for the users and customers involved. While these concepts have been loosely explored in academia, an empirical investigation into the methods and practices of industry leaders was nevertheless needed to illuminate this new field of inquiry. Subsequently, through a case study analysis of experience-centric design agencies and consulting firms this study aimed to uncover patterns in existing design methodologies leading to rich user experiences. This thesis addressed the following research questions:

R1: What are the components or elements that lead to rich user experience?

R2: What are the organizational behaviours and perspectives that must be adopted by design consulting firms shifting focus from the delivery of products and services to fostering rich user and customer experiences?

R3: What are the most effective design processes and practices being utilized by experience-centric design consulting firms that ultimately give rise to rich user experiences?

To examine the research questions the study followed a qualitative multiple-case study design as outlined by Yin (1994). The case study research strategy was chosen as it allowed for how, what and why questions to be answered. This allowed the researcher to gain a holistic understanding of real-life events such as organizational and managerial 
processes. Moreover, this type of research tool is well suited to exploratory investigations in which the variables are still unknown and the phenomenon is not fully understood (Meredith 1998). A case study design was therefore appropriate, as approaching the area of experience design from a system's perspective presents a new design space in need of further exploration.

Accordingly, seven case studies of design consulting firms offering experiencecentric services were completed. Semi-structured, in-depth interviews served as the primary source of evidence. One of the major strengths of case-study data collection is the opportunity to use multiple different sources of evidence (Yin 1994). This provides the study with converging lines of inquiry, offering multiple measures of the same phenomenon. Therefore, a qualitative document analysis on each firm was also completed in order to corroborate the evidence collected from the interviews. It is important to note that the term document serves as a catchall phrase to include such things as podcasts, books, journal and magazine articles, and website stories of the respective case firms. The narrative data from the interviews were transcribed and analyzed based on Auerbach and Silverstein's (2003) methodology, where relevant text is highlighted and grouped into repeating ideas. A cross-case analysis allowed the repeating ideas to be categorized into five patterns related to the research questions. Similarly the document analysis served as a secondary source of evidence and followed the qualitative media analysis research method as set out by Altheide (1996).

This chapter offers a detailed account of the research methodologies employed by the investigator in conducting the case study analysis. Firstly, the companies chosen for investigation are discussed, followed by a description of the research actions taken in performing the in-depth interviews and document analysis, respectively. Finally, steps to ensure the study's validity and reliability are discussed in the last section of this chapter. 


\subsection{CASE STUDIES}

\subsubsection{SAMPLE}

Due to the specialized nature of experience design the researcher chose a purposive sampling strategy. Based on the research questions, the ultimate goal of this study was to uncover design patterns or guidelines that could be implemented by other designers or corporate visionaries in industry. Therefore, the main criteria for selection involved firms which placed the user experience at the core of their business offering. Critically important was their ability to deliver these experiences across a multitude of business services such as product, system, service, retail, and/or UX design. Moreover, only companies with established expertise in delivering exceptional user experiences for their clients were selected, as they were most likely to provide the insights that would lead to a set of design patterns that could have broader applications in the design community. Lastly, as this area of design is relatively new, identifying companies which were able to provide access to key individuals with in-depth knowledge in the area, such as designers, strategists, or company founders was paramount in selection.

Companies were primarily identified based on public reputation. Furthermore, companies which had a global presence, were active in social media and contributed regularly to reputable publications were pursued. Expert opinions also served in helping to select appropriate design firms. Of the list of firms contacted, seven agreed to participate in the study. Table 1.0 provides an overview of the chosen design consulting firms and the services they provide. Additionally, Table 2.0 provides a description of each case company and the primary and secondary sources of evidence analyzed. Although this study is not situated within the realm of $\mathrm{HCl}$, it is important to note that several companies chosen for investigation had a solid UX or UI design foundation. This may be due to the fact that when selecting case companies, those with a strong 
understanding of experience were predominantly UX based. However, the context of the study was clearly established and provided to interviewees prior to beginning interviews and therefore, should not affect the outcome of the study. Nonetheless, this topic is revisited in Chapter Five, under research limitations.

Table 1.0 Description of Sample: Design Consulting Firms, Services and Locations

\begin{tabular}{|c|c|c|}
\hline Name & Services & Location \\
\hline IDEO & $\begin{array}{l}\text { Brand, Business Design, Digital Experiences, } \\
\text { Education, Energy, Engineering, Financial, } \\
\text { Services, Food \& Beverage, Health \& Wellness, } \\
\text { Organizational, Design, Play, Public Sector, Social } \\
\text { Innovation }\end{array}$ & $\begin{array}{l}\text { USA, UK, } \\
\text { Germany, } \\
\text { Japan, } \\
\text { China, } \\
\text { Korea, } \\
\text { Singapore, } \\
\text { India }\end{array}$ \\
\hline Adaptive Path & $\begin{array}{l}\text { User Research, Experience Strategy, Experience } \\
\text { Design, Implementation Support, } \\
\text { Mentorship \& Training }\end{array}$ & $\begin{array}{l}\text { USA, } \\
\text { Amsterdam }\end{array}$ \\
\hline Method & $\begin{array}{l}\text { Research and Design, Brand Identity, Digital Media } \\
\text { Design, Product User Interface, Print } \\
\text { Communications }\end{array}$ & USA, UK \\
\hline Akendi & $\begin{array}{l}\text { Brand Experience, Customer Experience, Business } \\
\text { Software UX Design, Web \& Customer Portals, } \\
\text { Mobile User Experience, Product Design, } \\
\text { Wayfinding Design, Usability Consulting, User } \\
\text { Experience Training }\end{array}$ & $\begin{array}{l}\text { Canada, UK, } \\
\text { USA }\end{array}$ \\
\hline Macadamian & $\begin{array}{l}\text { User Experience Design, Software Development, } \\
\text { Training, Research and Strategy }\end{array}$ & $\begin{array}{l}\text { Canada, } \\
\text { USA }\end{array}$ \\
\hline Ziba Design & $\begin{array}{l}\text { Product Design, Retail Design, Service Design, } \\
\text { Interaction Design, Communication Design, } \\
\text { Packaging Design, Brand DNA \& Identity, Product } \\
\text { and Service Innovation }\end{array}$ & $\begin{array}{l}\text { USA, } \\
\text { Germany }\end{array}$ \\
\hline Kicker Studio & $\begin{array}{l}\text { Service Design, User Experience Design, Sensory } \\
\text { Design }\end{array}$ & USA \\
\hline
\end{tabular}


Table 2.0 Description of Case Studies: Sources of Evidence Collected per Company

\begin{tabular}{|c|c|c|}
\hline \multirow[t]{2}{*}{ Name of Company } & \multicolumn{2}{|c|}{ Sources of Evidence } \\
\hline & $\begin{array}{c}\text { Primary } \\
\text { (interviews) }\end{array}$ & $\begin{array}{c}\text { Secondary } \\
\text { (Document Analysis) }\end{array}$ \\
\hline IDEO & $\begin{array}{l}1 \text { in-depth interview } \\
3 \text { on-site unstructured } \\
\text { interviews }\end{array}$ & $\begin{array}{l}\text { - About Us \& Services } \\
\text { Description } \\
\text { - } \text { Process Description } \\
\text { - } \text { Podcast by Diego } \\
\text { Rodriguez } \\
\text { - Website stories/case- } \\
\text { studies } \\
\text { - Published Article }\end{array}$ \\
\hline Adaptive Path & $\begin{array}{l}1 \text { in-depth interview (Peter } \\
\text { Merholz, Founder) }\end{array}$ & $\begin{array}{ll} & \text { About Us \& Services } \\
& \text { Description } \\
\text { - } & \text { Process Description } \\
\text { - } & \text { Blog Posts } \\
\end{array}$ \\
\hline Kicker Studio & 1 in-depth interview & $\begin{array}{l}\text { - About Us \& Services } \\
\text { Description } \\
\text { - } \quad \text { Process Description }\end{array}$ \\
\hline Method & 1 in-depth interview & $\begin{array}{l}\text { About Us \& Services } \\
\text { Description } \\
\text { - } \quad \text { Process Description }\end{array}$ \\
\hline Akendi & 1 in-depth interview & $\begin{array}{l}\text { - About Us \& Services } \\
\text { Description } \\
\text { - } \quad \text { Process Description } \\
\text { - White Papers }\end{array}$ \\
\hline Macadamian & 1 in-depth interview & $\begin{array}{l}\text { - About Us \& Services } \\
\text { Description } \\
\text { - Blog Posts }\end{array}$ \\
\hline Ziba Design & 1 in-depth interview & $\begin{array}{l}\text { - About Us \& Services } \\
\text { Description } \\
\text { - White Papers } \\
\text { - Core } 77 \text { Educational } \\
\text { Series }\end{array}$ \\
\hline
\end{tabular}




\subsection{PRIMARY (INTERVIEWS)}

\subsubsection{SETTING}

The in-depth interviews were conducted remotely via telephone, with the exception of three on-site, unstructured interviews completed in addition to an in-depth interview with employees of IDEO. The three on-site interviews took place in casual environments at the IDEO office in Palo Alto, California, including the lunchroom and a public meeting room. Prior to conducting any interviews, participants were contacted via email to assess their interest, availability, as well as to schedule a date and time for conducting the interview. As the telephone interviews were conducted remotely, the researcher called participants from a home office at the number and time of their choice. Some interviews were conducted during regular working hours, where participants were reached on their office phones, while others preferred to be contacted at home or on a mobile phone. Participants were located in either Canada or the United States, depending on their company headquarters.

\subsubsection{SAMPLE/PARTICIPANTS}

Based on literal replication logic, participants who shared similar professional backgrounds and experience were selected, expecting to lead to similar results (Yin, 1994). Due to the particularity of the experience design field, a purposive sampling strategy was chosen, as only experts in this area would be able to provide a great deal of in-depth knowledge regarding the best processes and practices necessary when designing for experience. The researcher also utilized a snowball sampling procedure. A letter of information explaining the background and purpose of the research was attached in an email to colleagues to help them in identifying suitable candidates (Appendix A). This research overview was then distributed to the potential participants in 
an email for their review. Any candidates interested in participating were prompted to contact the researcher directly. All contact, excluding the interview session, was made via email.

In order to understand the most comprehensive components of experience design participants who had a minimum of five years of professional experience in this field were requested. Interviewee job titles included CEO, founder, partner, creative director, systems designer and vice-president of strategy.

\subsubsection{MEASUREMENT INSTRUMENTS}

Semi-structured, in-depth interviews were conducted using an interview protocol. The semi-structured format encouraged an open discussion allowing themes to evolve naturally. Questions moved from the general to the specific in order to build rapport with the participant and avoid establishing a set, where the participant would be inclined to provide predisposed answers (Sommer \& Sommer, 1997). A list of ten to fifteen questions was asked based on the duration and direction of the interview. Consequently, at times some questions were omitted, while others were added ad-hoc during the course of the interview to delve deeper into a particular topic of interest when relevant. Furthermore, questions were asked in an open-ended format and focused mainly on the meaning of experience design, the research and design methods used, and perspectives of the respective firms. Probes were also used to further explain an abstract concept or obtain supplementary information on a certain question.

\subsubsection{DATA COLLECTION}

As previously mentioned, the researcher conducted telephone interviews for reasons of convenience, limited access and availability of participants, as well as the geographic distance between the researcher and the participants. A total of ten one-on- 
one interviews were conducted between November 2011 and January 2012. An interview script (Appendix B) and protocol (Appendix C) were drafted to aid in systematic data collection. Interviews began by providing participants a general overview of the study, helping to set the context for obtaining information relevant to the research problem. The interviewer also requested each participant's oral consent in identifying the design firm's company name in the reported findings. Participants were informed that their responses would not be attributed to them in any way and they would remain anonymous in the findings and thesis. In order to ensure accuracy of data collection, participants were also asked for permission to audio record the interview session. As the phone interviews were held with participants who live outside of Canada (i.e., United States), oral consent versus written consent was more appropriate. Each interview lasted between 30 and 45 minutes.

\subsubsection{DATA ANALYSIS}

Interviews were transcribed verbatim - however, hesitations and grammatical errors were not included as they were not significant in helping to answer the research questions. Due to the semi-structured and open-ended question format, data analysis involved a coding process to categorize and label the data into meaningful chunks. All analysis was completed using Microsoft Word. Interviews were analyzed based on Auerbach and Silverstein's (2003) book Qualitative Data: An Introduction to Coding and Analysis. The goal was to draw out major themes or relationships in the hopes of developing a set of design patterns. The research questions as well as the theoretical framework established in the literature review created a bounding box through which to approach the data analysis. With the latter in place, the researcher analyzed the transcripts individually before merging the comprehensive results from each. 
Firstly, from the raw transcript, relevant text was selected and transferred to a separate document. Relevant text is text that relates in any way to the research questions and theoretical framework (Auerbach and Silverstein 2003). Subsequently, the relevant text was scanned for repeating ideas. Repeating ideas are similar ideas expressed by two or more research participants (Auerbach and Silverstein 2003). Repeating ideas were first identified in the separate transcripts and then combined with the repeating ideas from all other transcripts. The coding process involved selecting a starter idea and moving that excerpt into a new document titled Repeating Idea 1 . Then the relevant text was searched for all ideas related to the starter text. This process was repeated until all unique ideas were drawn out and separated into their respective "Repeating Idea" documents. A master list of repeating ideas was created, where all repeating ideas assimilated from the individual interview transcripts were combined. At this stage, ideas were either amalgamated, re-grouped, or discarded as orphan text. In total, 48 repeating ideas were identified. A sample of a repeating idea with relevant passages is shown in Table 3.0. These repeating ideas were then grouped into coherent categories and organized into 15 themes. Table 4.0 provides an example of repeating ideas grouped into themes. The next step involved organizing the themes into patterns that would help in answering the research questions. In addition, Appendices $G$ through I provide supplementary samples of repeating ideas grouped into themes and themes into patterns. However, for reasons of client confidentiality as well as participant anonymity the full transcripts could not be provided. The process was designed to be transparent and systematic in order to ensure the validity of the results (Auerbach and Silverstein 2003). In sum, five patterns emerged and are discussed in detail in the following chapter. 
Table 3.0 Sample of Relevant Text Selections Grouped into Repeating Idea 1

Repeating Idea \#1: Customer experience means every way you touch that product or brand: "anything that a consumer touches that is related to your company or your brand is part of that experience"

a) "The way in which products and services are designed to be experience [...] across touchpoints (P4, p.1)."

b) "the experience is every way in which the end customer experiences and touches the product that goes way beyond the actual UI (P1, p.1)."

c) "Where the experience is every way you experience that product from the moment you buy it and get it and purchase it and unpackaged it, your experience purchase it. The most common and overused example is the iPod versus other music players where what Apple did was realize that the experience was also how you discover, purchase and consume the music - it wasn't just the click wheel on the device which related to the design engineering. It went way beyond that and that's what made it so successful (P1, p.1)."

d) "Experience design means a consideration of design more than experience beyond just the point that you interact with something so you can talk about how someone interacts with a piece of software or an object but actually considering how this all fits together in a wider experience so the piece of software exists as a part of a workflow. What does that workflow look like? An object intersects to serve a particular task, maybe a task you perform before or after it. Technology exists; you get an object out of a piece of packaging. The experience of opening the packaging also contribute to the experience that you have with the object itself. So its really a case of not just concentrating on one particular instance of interaction but going beyond that and seeing how these interactions get together and form a whole experience (P3, p.3)."

e) "I started to realize the power of true consumer experience and how that all worked together - to me that means all of these things, anything that a consumer touches that is related to your company or your brand is part of that experience. (P2, p.2)." 
Table 4.0 Sample of Repeating Ideas Organized into Themes

\section{Theme \#1: Every Touchpoint Counts}

R1. Customer experience means every way you touch that product or brand: "anything that a consumer touches that is related to your company or your brand is part of that experience"

R2. Customer experience as a whole is mitigated or enhanced depending on the experience at each touchpoint: "everything that touches the product either adds or takes away from the experience as a whole"

R3. Holistic picture of everyone involved (not just the end user)

Theme \#2: Realizing Where You Fit

R4. Understanding the user holistically and how each touch point within the chain of events serves them: "A holistic approach to design that takes into account the whole person" - human centered cognitive

R5. Hiring big picture thinkers: "It helps to have big picture thinkers on the team" R6. Acknowledge other parts of the chain even if you are only designing one channel: "Understanding how that fits in with a larger ecosystem"

R7. Experience unfolds within a wider narrative: "Realizing that it exists within a wider narrative that unfolds when people start to experience a brand"

\subsection{SECONDARY (DOCUMENTS)}

\subsubsection{SAMPLE}

As previously mentioned, a document analysis was carried out to augment the evidence obtained through the in-depth interviews (Yin 1994). The research questions helped in informing the most appropriate units of analysis to determine which documents should be investigated. The units of analysis included About Us and Service descriptions from the respective firm websites, as well as blog postings, white papers, articles, process descriptions, and any other relevant material. These documents were selected as they were expected to most accurately reflect the perspectives, philosophies, and practices of the individual case firms. It is important to note that not all of the documents described were available from each firm. Appendix D provides a summary of the design 
firms and the units of analysis that were selected from each. The analysis of the interviews led to five design patterns, which served as a lens through which to collect and analyze documents from each case organization. A theoretical sampling strategy was used, where materials were selected for being conceptually relevant to those five patterns. This helped in narrowing the possible documents for investigation.

\subsubsection{MEASUREMENT INSTRUMENTS}

Based on the five patterns identified from the interviews a protocol was prepared to guide the data collection process. The protocol evolved over several drafts as more documents were collected and was amended to include all suitable topics. Once all adjustments were finalized the protocol was assessed to see if the conceptual problem was adequately covered by its themes. The final document protocol (Appendix E) included case number, title, medium, date, author, main topic, a brief description of the document under investigation, source and contained the five patterns as categories for analysis. It also included a reflective segment in which the researcher could comment on the relevance of the selected passages or highlighted concepts from the text.

\subsubsection{DATA COLLECTION}

With the units of analysis serving as a preliminary guide, case firms' websites were visited and webpages pertaining to their mission statements, services and processes were saved. Within their websites, links to their blogs, whitepapers or any other relevant material were investigated. In most cases, these were visibly displayed as direct links from the home page. Largely, specific blog posts and whitepapers concerning the five key patterns could be uncovered using a generic web search functionality embedded within the corresponding webpage. In order to uncover the most pertinent material, the patterns were used as search terms. As well, other more general 
searches included key words such as "experience + design" and "systems + design". As more documents were found and read, more search words were identified and helped to expand the theoretical sample.

Whenever possible PDFs or screen captures were taken and saved electronically. The data were managed using Microsoft Word and stored in electronic folders organized per firm and unit of analysis. Each document's completed protocol was stored in the same folder under the following file name: DP_title of document.doc.

According to Altheide (1996) 15 to 20 documents provides a sufficient sample size as qualitative content analysis most often focuses on the range of meanings, themes and processes. In total, 23 documents were analyzed. They included, but were not limited to podcasts, videos, whitepapers, and website stories. Once two thirds of the documents had been analyzed the files were reviewed and searched for examples of missing or underrepresented categories, at which point adjustments were made to the sample. Detailed notes were kept on retrieval dates and evidence as most of the documents were gathered from online sources. Documents were thoroughly read and relevant text was highlighted and added to the protocol under each category - capturing many descriptive examples and using direct quotations whenever possible. Key words were used to further categorize collected data under themes relating to each pattern in order to expedite the data analysis.

\subsubsection{DATA ANALYSIS}

As formerly stated, the document analysis followed the research methods outlined by Altheide (1996) for qualitative content analysis. The goal of the analysis was to associate the meanings of each document with the conceptual issues raised during the interview analysis and were mainly used to supplement understanding and interpretation from those sources (Yin 1994). Data analysis began by carefully reading 
and listening to all documents and categorizing their content based on the five patterns previously identified. In order to aid the researcher in becoming more familiar with the data, comprehensive notes were taken throughout and mini-summaries were provided on each topic added to the individual document protocols. Once all documents had been thoroughly investigated and their content broken into the patterns using the protocol, a new Word file was created for each pattern. Subsequently, the relevant passages and mini-summaries for each pattern from the respective documents were amalgamated within each new Word file. The integrity of the company and source information for each passage was maintained so as to facilitate report writing. The dedicated Word file for each pattern aided in comparing and contrasting the findings from each document investigated across case firms. These findings were then integrated with the outcomes from the interviews to ultimately form the seven case studies on experience-centric design firms.

\subsection{VALIDITY AND RELIABILITY}

To increase the validity of the interviews a pilot study was conducted with one participant to ensure that the appropriate questions were asked and identified insights relevant to the research problem. Once the pilot study had been conducted the answers were analyzed and resulted in a few minor changes to the interview protocol. Prior to beginning, the interview protocol was reviewed by an expert and the necessary adjustments were applied.

Throughout both the collection and analysis of the documents and interviews, steps were taken to ensure the validity of the qualitative findings. Detailed records were kept and the researcher maintained a strong chain of evidence by separating each analysis step into separate Microsoft Word documents. In doing so, it is possible to 
follow the derivation of any evidence starting from the raw text all the way to the five emergent patterns. Moreover, construct validity was addressed by using interviews and documents as multiple sources of evidence. Also, when reporting the results, footnotes and citations of specific documents and interviews were used. Similarly, thick descriptions, imparting the feelings, actions and meanings of the participants through quotations (Bui 2009), were provided of each pattern to paint a picture of the concepts. In order to increase reliability the thesis supervisor was asked to analyze three of the transcribed interviews based on the categories developed by the researcher for coding. Subsequently, both individuals came together and discussed the results and the appropriate changes were made to the coding procedure. A case-study database also was created where an annotated bibliography (Appendix F) was kept of all documents analyzed and a hierarchy of importance was established for each. 


\section{CHAPTER FOUR}

\section{RESULTS}

The seven case studies on experience-centric design firms provided a wealth of knowledge to the researcher in regards to the design processes and methods used in practice when designing for rich experience. From the case studies five patterns emerged as being particularly relevant. They involve holistic thinking, building emotional connection, harmonizing touchpoints, interdisciplinary collaboration and client empowerment. Each section of the results chapter is dedicated to a cross-case pattern, where the individual cases are dispersed throughout and summary information is presented in abbreviated vignettes. The choice to present the results based on patterns versus individual case studies was appropriate as the purpose of the research was not to portray the process of any single firm, but rather to synthesize the lessons from all of them.

Therefore, thick descriptions (Bui 2009) are provided for each pattern using key passages from interviews, as well as key pieces of evidence from the document analysis. The latter helps to paint a picture of the findings and set the context of the concepts presented.

\subsection{HOLISTIC PERSPECTIVE}

The design firms under investigation embraced a holistic perspective when it came to designing for experience. This necessitated a constant regard throughout the design process of all contexts, cultures, people, products and services impacting the experience. Particularly, when asked to describe the meaning of experience design 
almost all participants responded that it involves a consideration, beyond one interaction, to how all the interactions fit together within a broader experience. In describing this concept one participant stated,

"The experience of opening the packaging also contributes to the experience that you have with the object itself. So it's really a case of not just concentrating on one particular instance of interaction but going beyond that and seeing how these interactions get together and form a whole experience."

Consequently, participants went on to express that the experience as a whole is mitigated or enhanced depending on the impression of each touchpoint. The example of a long, complicated and unpleasant customer service call was offered in clarification. Explaining that as a result of this frustrating interaction, the user's entire experience would be diminished, regardless of their prior relationship with that company. In the same vein, one designer elucidated,

"it involves so many things, it's not just the aesthetic or your first reaction, it's every single interaction you have with the whole chain of events that lead you to experience the product, how you can have an emotional reaction to every part and it can lead to good or bad emotions along the way."

In order to accommodate for this, a holistic perspective is required by the design firm and subsequently, by its' team members. The firms described their employees as individuals who are inherently good at understanding the larger system and suggested hiring big picture thinkers. To this point, team makeup was a topic that persisted throughout the case studies and as such, is treated as a separate pattern further on.

Similarly, they stressed that their ability to create memorable user experiences rested on their understanding of how and where their offering fit within the broader chain of events or customer journey. This involved acknowledging all other parts of the chain, even if they were only designing one channel. One participant described it as, 
"understanding what the ecosystem of touchpoints are and how they work together. So even if you're only working on one piece of that - understanding how that fits in with a larger ecosystem, within that brand experience."

Diego Rodriguez (2006), a partner at IDEO, provided further evidence of the importance of ecosystems in a podcast for a Core77 Discussion. He explained that great experiences require ecosystems and must be designed for the context in which they live. Therefore, by recognizing the interconnected system the case consultancies were better qualified to deliver the appropriate experience for the touchpoint they were designing. In contrast, one participant explained that overlooking this would adversely impact the experience:

"What happens is if you don't acknowledge that concept you can overload the web experience or whatever channel you're working on. You tend to overload it with the entirety of the service."

The latter also avoids designing a product or service within a vacuum, impervious to outside factors (Vossoughi 2010). Correspondingly, IDEO's Fred Dust and llya Prokopoff criticized the infamous Segway as a product that fell victim to ignoring the total context in which it existed:

“The two-wheeled transporter didn't catch on for lots of reasons, from cost and practicality to a mountain-high learning curve for use. Still an overarching issue was that it wasn't [emphasis added] intentionally designed to be a part of a larger-scale system; rather, it was an individual product at odds within a larger ecosystem." $(2009,53)$

Moreover, participants explained that pivotal to designing for experience was the understanding that the experience a user has with any part of an organization ultimately sits within a wider narrative. Thus, designers recognized that each user has a life story and considered how the product or service they were designing fit into the broader narrative that is the user life. 
Interestingly, the document analysis revealed a strong opposition towards designing in silos - mentioned in multiple blog posts, videos, and whitepapers (Farnham n.d.; Newbery n.d.(a).; van Gelderen 2010). Here, the term silo refers to different functional groups, or departments within an organization working in isolation from one another. Dust and Prokopoff (2009) go on to explain that "functioning 'silos' can be effective at a particular task, but the overall system eventually threatens to bottom out or limp along. It is only by combining components into a whole that we see the potential for a solid, sustainable ecology" (p.55).

Participants also described how experience occurs over time and in particular, through three phases: Attract, engage and extend (Dubberly and Evenson 2008). The attraction phase refers to how the user is drawn in, whether through the website, advertising, or word of mouth. This is followed by an engagement phase, when the user is interacting directly with a core service or product and lastly, the extension phase. Notably, the case firms were most concerned in the third phase and differentiated themselves by their ability to extend a user's experience beyond the lifecycle of one product or service. They were constantly thinking about how to keep the user coming back and engaging in other parts of the company to enhance their collective experience.

Participants stressed that a holistic perspective is something to be carried through all phases and approaches of designing for experience. This also requires a holistic picture of everyone involved, not only the end user. As such, it must take into account the whole person - appreciating the user as a complex, multi-faceted human being. Ziba believes, "our job is to ensure that the characters are not lazy caricatures, but believable people with emotional and functional depth" (Backett 2011, 5). The latter denotes a theme reiterated in all conversations across several different interview topics, and is discussed in detail in the upcoming section. 
In brief, Vignette 1.0 provides a summary of the ideas identified through the case study analysis supporting a holistic perspective for teams concerned with the design of rich user experience.

Vignette 1.0 Philosophy Behind the Holistic Perspective

A holistic perspective involves:

- A consideration, beyond one interaction, to how all the interactions fit together to form a whole experience.

- Understanding that experience as a whole is mitigated or enhanced depending on the impression of each touchpoint.

- Recognizing the ecosystem attached to your offering to design the appropriate experience for that touchpoint.

- Avoiding designing in or for functional silos

- Experience occurs over time, through three phases: Attract, Engage, Extend.

- A holistic picture of everyone involved, not only the end user.

\subsection{BUILDING EMOTIONAL CONNECTION}

Here the concept of empathizing with the user who is utilizing a human centered process in order to build an emotional connection between the user and the product/service or system being designed is discussed. Although a human centered approach is not a new concept in design, it was a quality of experience design that case participants were adamant in expressing and as such, warrants attention. In fact, one participant stated plainly, "everything we do is human centered design." The emphasis on human rather than user centered stems from applying a holistic perspective in understanding the user as a complex being.

Through the case-study analysis a distinction was made between actionable items within the design process and a more cognitive connection to the user resulting from these activities. Ultimately, this allowed the design team to build a product, service, 
and/or system to which the user was emotionally connected. Therefore, this approach is divided into two applications: Human centered activities and embracing the user perspective resulting in an emotionally charged product or service. Human centered activities are equal to the traditional understanding of human-centered design - wherein users are consulted and continually involved at each phase of the research, design and development process. While embracing the user perspective involves building team empathy and understanding the user's latent needs. This section will focus more on the latter, as it is an underdeveloped phenomenon in the field of design.

\subsubsection{HUMAN CENTERED ACTIVITIES}

First and foremost, human centered activities began with in-depth, immersive research. Interviewees referred to in-depth research as the keystone of good experience design, forming the foundation from which to build their project. Participants believed that,

"preparing and front loading your work is the singular most important step. [...] This is a thing that can be really frustrating to [our] clients who haven't worked with us before. There's a lot of stuff that needs to happen up front, and I'm really a strong advocate in our research methods and our ability to dig deep and get down at the latent consumer needs - their emotional needs."

As compared to design firms which lack an experience-oriented philosophy, the case study firms differentiated themselves by the level of depth in which they approached and conducted their research. Participants made a significant distinction between types of research and focused mainly on ethnographic research methods. Chiefly, observations, shadowing and "day-in-the-life" activities were said to be the most effective in uncovering the user's latent needs and identifying design opportunities. While interviewing, surveys, and focus groups were also used, observational research was nonetheless said to lead to the richest insights. It was also stated that one of the 
major reasons for conducting this research was to gain a deep understanding of the contexts of use:

"The cornerstone for everything we do is contextual research and really understanding what context the products and services we're designing will be used in and the variety of contexts it will be used in across time and then really looking at how, what people want and need from a particular experience/brand in that context."

What is more, they spoke of iterative prototyping throughout the project, both high and low fidelity, and soliciting plenty of user feedback. Almost all participants described a process where the user was involved in every phase: During research, prototyping and testing, and/or design workshops.

\subsubsection{EMBRACING THE USER PERSPECTIVE}

The activities mentioned above were profound in helping the team to embrace the user's perspective. Once they had collected all the insights from the in-depth research their design teams were able to gain a holistic picture of the users' needs and how they could begin to serve them. One participant explained,

"because we really studied a day in the life of this one customer we really understand how this can be important to them and how it can solve some kind of need, pain or desire and we have empathy for that customer/user."

Moreover, firms explained that by involving the user in their design and research phases they were able to build empathy across their team members. Empathy appeared to be the magic ingredient behind the human centered approach and a critical component of knowledge production. As such, many participants stated that they aimed to hire innately empathetic individuals. On this subject, it was fervently expressed that, 
"literally "understand the customer needs" mindset is crucial. So one of the most important things we can do, I could do, is make everybody be able to have some empathy for our customer. [That way] they can't help but do great work for them [...] When you have that empathy you can appreciate what are better design solutions inherently because you can put yourself in your customers shoes better and so the research act and how you communicate the results of that research are crucial."

Subsequently, a tool often used to understand and communicate research findings in order to build this empathy across teams and clients was storytelling. When participants spoke of using stories, such as personas, scenarios and storyboards they stressed that they should be factual, based on real-world circumstances:

"So this is all within a story, so I think all experiences will be embedded within a story, and I think the more you acknowledge that and the sooner you rely on real stories and not just made up ones. But rather ones that we observe, your products and your end design will be far richer to some people and they will be able to resonate with it on a far more profound level."

Paramount to experience is emotion - one cannot exist without the other. Therefore, a recurring goal by many of the participants was to elicit positive emotions from the user in order to achieve rich experiences. In describing the importance of emotion one participant referred to Starbucks as the leading standard:

"So you're looking at thousands of percent of increase of the value there between the commodity and the experience. And that's because there is some emotional magic that happens there - the value add is really that emotional connection."

As such, creating rich experiences started with centering the design process around the user. Human centered activities, such as in-depth, ethnographic research, iterative prototyping, and involving the user in all phases resulted in a team able to empathize with the user. This mentality allowed them to gain a deep understanding of the user as a whole complex person with pains, needs and desires, thereby helping to identify unique design opportunities in order to build an emotional connection between 
the user and the product, service, and/or system. Vignette 2.0 provides a summary of these concepts.

Vignette 2.0 Building an Emotional Connection to Foster Rich Experience

Building an emotional connection between the user and product/service/system involves:

Human Centered Activities

In-depth research

- In-depth research forms the foundation from which to build a design

- Utilize immersive in-depth research techniques, such as ethnography and observation.

\section{User involvement and feedback}

- Involve the users throughout the entire process

- Soliciting user feedback through high and low fidelity concept testing and iterative prototyping throughout

Embracing the User's Perspective

\section{Building Empathy}

- Have all project members (including client) develop empathy for the user through team involvement in all phases and real-world stories

\section{Understanding user's latent needs}

- Understanding the user's emotional latent needs; their pains, needs, desires

- Prioritize user needs

\subsection{INTERDISCIPLINARY COLLABORATION}

The interviews and subsequent document analysis uncovered numerous instances where firms paid close attention to their team's composition and noted the importance of teamwork. In fact, they attributed much of their success in the field of experience design to having team members from different professional backgrounds with varying perspectives, collaborating to solve a common problem. Thus the concept of the "lone" or "genius" designer was obsolete. Participants spoke to creating cross-functional 
teams, collaborating tightly from beginning to end, whether they were anthropologists, sociologists, interaction designers, mechanical engineers, and/or product designers. The latter was reiterated during the document analysis. For instance, an analysis of Ziba's home page revealed that the firm boasts individuals from 42 fields of experience, 25 spoken languages, and 18 nationalities (Ziba Design $\mathrm{n}$. d). In describing their project team model, one participant noted,

"They work in this group environment across different disciplines. And this is the other thing; you don't just have hard-core designers, but you have people who are anthropologists or education background people, or some people who are computer geeks and we all get together and we're exchanging and cross-pollinating our ideas."

Equally, the UX group at Macadamian holds weekly design critiques where everyone is welcome and anyone can present a project to benefit from the feedback and outside perspectives of other designers, researchers, or engineers. Appreciably, participants emphasized the positive impact of hiring people capable of "wearing multiple hats" or in other words capable of assuming multiple roles within a project. These individuals were referred to as design generalists, a term used by two of the participants, but a concept expressed by many. Chiefly, one participant explained,

"On the ... project we had an additional interaction designer, design researcher, UX person and an intern who would do a lot of work on visual design but they all did kind of everything. It was more the emphasis, the researcher would be the one who shot the photos for the storyboards and the interaction designer was involved with the research as well. He was the floater; he was active on both of the two tracks of the project." 
Furthermore, corroboratory evidence was provided by examining IDEO's career website, where several job postings described interdisciplinary ${ }^{2}$ or generalist roles and responsibilities (IDEO 2012). Likewise, in a short video titled How to Be a Successful Designer, Robert Murdock, a principle of design at Method stressed that their most successful projects emerged when designers were willing to work on challenges across disciplines (Murdock n. d.).

Moreover, these firms advocated high team involvement in all phases. They argued that firsthand knowledge cannot be substituted. Therefore, designers should partake in research activities and vice-versa. The latter also helped all team members build empathy for the user, as they have witnessed the problems directly. One participant favoured small generalist teams, claiming that big teams can lead to confusion and lack of accountability. It was also stressed on several occasions that "hand-offs" between siloed groups in an organization should be minimized wherever possible. Claiming that hand-offs between phases and functional groups led to the loss of insights and momentum. One participant reasoned that

\begin{abstract}
"it's easier to get a great customer experience when the whole team is collaborating tightly and is aligned on their goals and works side by side throughout the entire project. One of the hardest things to do in creating a great customer experience is if you've got to hand off designs, because anything can happen at that part. It's hard because even if you're handing off a concept design to a details design team, just imparting all the context for why certain decisions were made and not being involved. I think that's one of the biggest destroyers of experience is when it's been designed and developed by completely different people or different agendas."
\end{abstract}

\footnotetext{
${ }^{2}$ Interestingly, most of the participants spoke of building "cross-functional" teams. However, their descriptions of team models related more closely to an "interdisciplinary" approach. Interdisciplinarity involves the tight collaboraticon of team members throughout a project and is defined as "an approach to a particular problem space using integrated outputs from combined collections of methods that owe to combined domains of expertise (Blevis and Stolterman 2008. 4)."
} 
One of the methods they spoke widely of was creating dedicated project spaces and collocating team members for the duration of the project, or at the very least during the early stages of the project. They believed some of the benefits in doing so were fast turn-around time, quick ideation, and project immersion. To illustrate this point, consider the following interview passage:

"We try to set up spaces for each other for our projects so that our teams can work within those spaces, from the start of a project right through to the end. Because I think, only when we truly collocate people in a space do they begin to adjust to each other's rhythm and begin to generate really great stuff together."

From the case study findings, Vignette 3.0 provides a summary of the ideal approaches that aid in building a team dedicated to designing rich experiences.

Vignette 3.0 Human Resource Allocation for Rich Experience Design

1. Build teams with good chemistry that best suit the design challenge.

2. Create teams of individuals from varying functional backgrounds and perspectives.

3. Hire design generalists capable of taking on multiple roles in or across projects.

4. Minimize hand-offs by having team members collaborating tightly throughout entire duration of the project.

5. Collocate team members in a designated project space.

6. Involve all team members in all phases of the project.

\subsection{HARMONIZING TOUCHPOINTS}

During the interviews, several participants considered the principal factor contributing to rich user experiences as designing across touchpoints. The latter was also reinforced through the analysis of the design agencies' respective websites, blogs, and publication materials (Adaptive Path 2011b; Matthew Hately, Macadamian Blog, entry posted August 18, 2011; Ziba Design n. d.). They advocated that their ability to 
deliver across touchpoints consistently made them powerful within the domain of experience-centric product, service and system design. Explicitly, when asked the reasoning behind their success one firm responded,

"If you're looking for experience design you should come to us, because we are looking at what's happening, what technologies you have, what the brand is, taking the pulse of the people that are out there. Looking at the pulse of the people who are buying watches, looking at materials, looking at finishes, looking at the packaging, looking at the brand promise that would show up in advertising. Looking at the point of sales display. Integrating across all those touchpoints, that's where we get our strength."

They went on to express that this could only be achieved through a team of individuals with a broad-reaching skill set. Not be confused with the concept of a "super hero designer", it is the idea that they have individuals from multiple different functional backgrounds capable of tackling the requirements needed in designing each touchpoint - further emphasizing the importance of building interdisciplinary teams. Similarly, the significance of harmonizing touchpoints was apparent across several of IDEO's projects, chiefly, their redesign of Air New Zealand's long-haul travel experience. IDEO's team rethought the "entire experience, from the cabin's layout and equipment (such as...seating) to the in-flight service and entertainment" (IDEO 2009).

As the topic evolved, a new theme emerged: Where fundamental to their success was their ability, not only to deliver across multiple touchpoints, but to do so consistently. This issue is considered in a whitepaper written by Akendi on the experience lifecycle: "Each experience is as important as the next time it occurs. For this reason, it is critical to have a cohesive plan that addresses all types of experiences throughout the experience lifecycle" (van Gelderen 2010). The notion of consistency was extended to a company's brand story. The design firms spoke of consistently telling the brand story and using it to help tie all of the touchpoints together. Decidedly, one participant remarked, 
"I really feel that its all about these storylines and trying to make sure that you are consistently telling the story of what your brand represents and how it makes its way through packaging, product, your website, your point of sales display, the clothes that your sales rep wear - so it's a pretty holistic concept."

The same participant offered the example of Apple, frequently cited when on the topic of experience design (Merholz et al. 2008). Exclaiming that Apple has done a very good job at consistently communicating their story, whether it be through the signage on the door of the retail environments to the packaging of their manuals.

Firms presented specific tools and methodologies in helping to ensure this consistency. Specifically they argued that good experience design requires detailed execution of each touchpoint. In explaining this idea, one participant used Virgin America as a leading example:

"On board the planes are different, the lighting, the entertainment system, the way the safety video plays, all these little touches that they have put a lot of thought into to make a coherent and very highly branded and very interesting experience."

Moreover, participants noted that the end experience is better served the more they are able to interact during the development and refinement stages. For one participant this meant staying involved with the client during the execution phase, to review the implementation and make any necessary tweaks to the design. Participants recognized that as consultants they are ultimately working within a set of constraints, and the scope of their contracts do not always include working over multiple touchpoints. Nonetheless, five of the seven firms presented practical solutions to help overcome these limitations in the hopes of impacting the service at a larger scale by aligning all team members and functional silos to a common vision.

In order to do so, one participant described the idea of a "North Star". Figure 4.0 depicts a quick sketch that was drawn during the interview to help clarify. They explained 
that every project should have a North Star (or design vision) guiding all functional silos and team members to a common end, ensuring a consistent experience across touchpoints. It was explained that this vision must be broad enough to encompass all touchpoints, flexible enough to be applied to each and individual enough to capture the buy-in of stakeholders, so they too can apply it and feel empowered. The North Star was described as a toolkit to reel in concepts that have gone astray and would utilize a set of overarching design principles or guidelines as its primary means of alignment. Likewise, many of the other design firms reported using design principles early on in the project to help align the team with the overall vision.

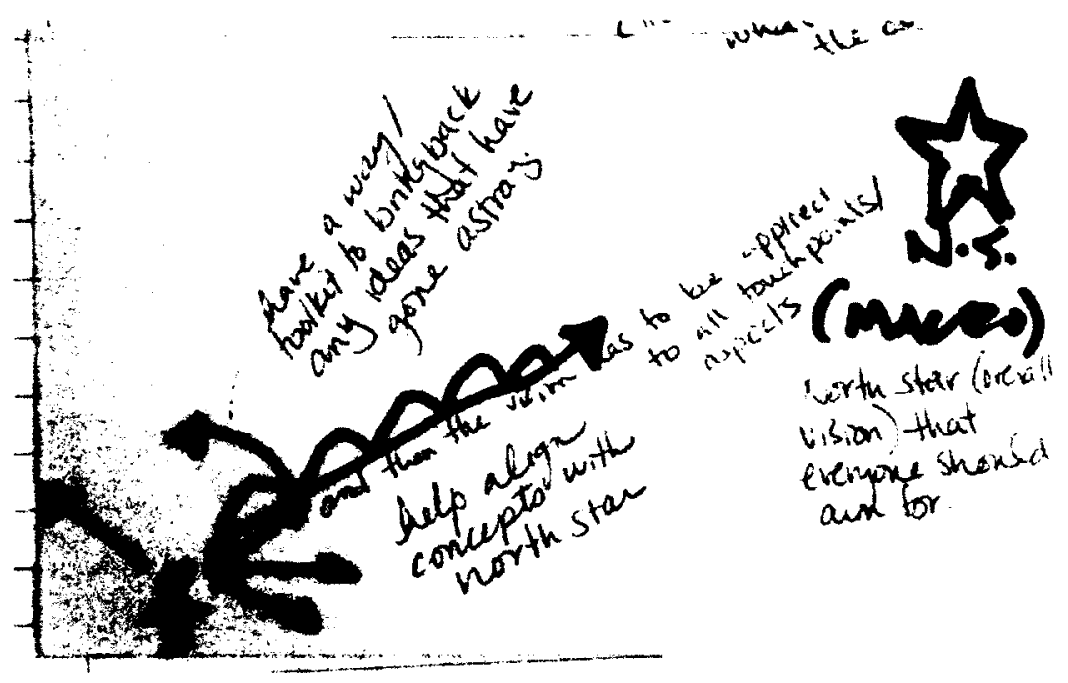

Figure 4.0. North Star. A picture of a sketch drawn by one participant during an on-site interview to help in expressing the concept of the North Star to align all touchpoints and functional silos.

Other examples of tools that some of the case firms employed included using design themes. For example, at Ziba teams build design themes during the early phases of a project that govern how each touchpoint will be designed: Its "feeling". Paul Backett, Ziba's lead industrial designer described design themes as "a set of carefully curated products, attributes, materials, and details that tell a clear story $(2011,7)$." Similarly, Adaptive Path talks about using design principles, personas, or value propositions as a lens through which to design the experience across multiple touchpoints (Chris Risdon, 
Adaptive Path Blog, entry posted November 20, 2011). One participant stressed that the design vision should communicate three concepts to everyone involved, including the client. They involve, the essence of what the product/service/system should be, its personality or DNA and the principles describing the brand and company.

To summarize, Vignette 4.0 presents an overview of the approaches and concepts introduced for harmonizing touchpoints in order to foster rich user experiences.

Vignette 4.0 Harmonizing Touchpoints for Rich Experience Design

Consistency

- Deliver across a multitude of touchpoints

- Integrate the experience consistently across all touchpoints

- Present a coherent brand story tied seamlessly across all touchpoints

Detailed Execution

- Strong attention to detail and careful execution of each touchpoint

- Involvement during the "refinement" stage to help implementation on client's end.

\section{Common Vision}

- Impact the service at a larger scale by aligning all team members and functional silos to a common vision using overarching design principles

\subsection{CLIENT EMPOWERMENT}

Beyond aligning all project members to a common vision, participants spoke of empowering their clients as a guarantee for impacting the experience on a larger scale. Participants recognized that ultimately, as consultants, they are handing off a design to their client and the responsibility to implement, sustain and grow the design vision shifts from the designer's hands to the client's. Consequently, these firms acknowledged that their success is intertwined with that of their client's. As such, they take on considerable effort to empower their clients throughout the design process so as to engender a sense 
of ownership, hopefully affording them the ability to take the design and, as one

participant aptly noted, "make it fly". Sohrab Vossoughi, Ziba's CEO and founder stated,

"Design beautiful experiences for your client and they will achieve one-off success; teach your clients how to find beauty for themselves and they will have the capacity to continually deliver truly beautiful experiences that can turn customers into long-term loyalists. (Vossoughi 2010, 4)"

Firms aimed to empower clients to bring the design in-house and learn to maintain it. They stated that experience design should become embedded in the client's business as it is an essential component of their business. Notably, in an interview, Peter Merholz, a founder of Adaptive Path, described the complexity of experience design,

"It's not a thing they can buy. You can try to buy the design from somebody but how is it going to live on? Are you going to have this agency forever to try to maintain it? You have to bring it in house to maintain it and make it grow and you need to feel that you own it and you need to feel that you are part of the process. [...] I think with experience design it is a different kind of design service that requires, in order for it to live, the client needs to understand it and embrace it and make good with it. "

The case companies offered some tools and techniques to help facilitate this dissemination. When asked about the most effective tool to help clients in extending the experience and applying it, one participant responded:

"Involvement in our process. Not to be a simple receiver of deliverables, but to be an active participant in our creation so they understand why decisions were made."

Participants noted that one of the biggest challenges they faced was in getting clients to trust the process. Subsequently, they explained that involvement in the process created vested partners, helping with enrolment and also building confidence. Likewise, participants mentioned that involving the client as soon as and as much as possible, 
allowed them to empathize with their user or customer. Once again highlighting the importance of a human centered approach. One participant remarked,

"A lot of times we'll have our clients build with us and at the same time experience the build. So at the same time see what their end users are experiencing. Doing that allows them to empathize, in which case now they're being impacted as well."

Similarly, in describing the importance of client involvement another participant referred to the familiar Chinese proverb: "Give a man a fish and you feed him for a day. Teach a man to fish and you feed him for a lifetime." However, this did not connote that clients became expert designers, rather that they gained a sensitivity towards making design decisions and implementing them. Several of the firms expressed analogous views, whereby clients have shifted from being idle receivers of deliverables to active participants in the research, design and development process. Markedly, Adaptive Path lists "Client empowerment. Through collaboration" as a major reason to hire them (Adaptive Path, 2011c). Firms noted this transition as a new movement in the design field, shifting away from the conventional "curtain" model where designers would unveil their design at various project milestones, using presentations and research reports as validation for design decisions. In this new approach, participants explained, clients participate during observation, testing, and they will also be brought in during strategic design workshops. Correspondingly, an element to emerge from the document analysis was the emphasis placed on training clients. In fact, four of the seven firms offered workshops and training in their list of services (Adaptive Path 2011a; Akendi 2012b; Macadamian 2012; Vossoughi 2010).

Part of this required communication between the client and the design consultants. Although the majority of these firms have moved away from typical design deliverables they did describe several techniques to be used when communicating to 
team members or clients using tangible artifacts. Key to their success was that the deliverables were actionable. They suggested that by creating deliverables that capture the design intent in a clear and concise manner, clients would be better able to take the design and execute the appropriate steps. Particularly, this meant using less wordy reports and presentations and using concepts and prototypes as support material.

Consider the following passage:

"If our deliverables are not actionable by our clients in some way then we are probably not doing our best job for them. And so one of the things that on my side we've been trying to do is make our presentations shorter, more clean, more distinct and really focus on the actual deliverables that the client cares about. [...] clients come to us for deliverables they want something that they can actually take action on - that's how you make your internal clients winners. They can take something from [us] and start to figure out how to make it."

Furthermore, participants felt that in order for research data and information to be salient, it must be represented in a visual way. Several examples were provided, such as building two by two population segmentation frameworks or experience maps. In a blog post by Adaptive Path, they described the process of creating an experience map for Rail Europe (Figure 5.0) and the advantages it brought to the project (Chris Risdon, Adaptive Path Blog, entry posted November 20, 2011). In doing so, the client was able to visually see the gaps and opportunities.

Moreover, the document analysis revealed a concept that eluded the interviews a central purpose behind using visual communication tools, as well as storytelling, was as a generative tool, or catalyst from which to inspire conversation and build on ideas (Backett 2011; Chris Risdon, Adaptive Path Blog, entry posted November 20, 2011). 


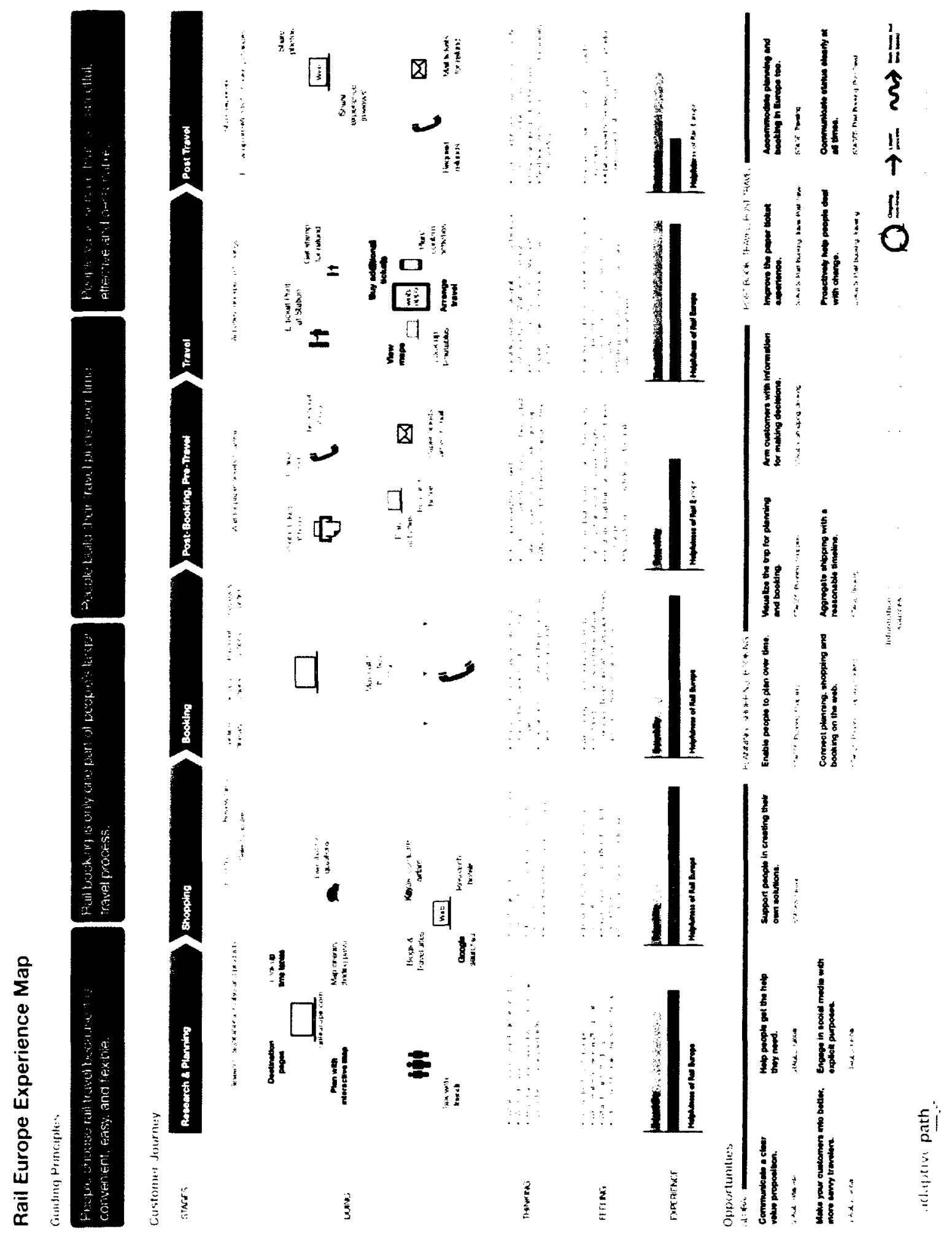

Figure 5.0 Experience Map. Depicts an experience map created by Adaptive Path for the Rail Europe user experience. (Chris Risdon, Adaptive Path Blog, entry posted November 20, 2011) 
Additionally, there was a strong association between designing for experience and storytelling as a means of communication. As previously mentioned, when using stories it is crucial to create them based on real-world data. However, beyond using realworld data, stories were often described by participants as being particularly useful in building empathy among clients and helping them to understand the reasoning behind the design.

For example, to illustrate the everyday challenges wounded war heroes face, IDEO created a heartrending video montage of three real life military families, struggling with those issues (IDEO 2010). Comparatively, in one project, Ziba had clients act out scenarios for how each product would be used in context as a sort of theatrical presentation (Vossoughi 2010). Other examples of storytelling included experience scenarios, personas, videos, and storyboarding. In particular, storyboards were described as being helpful in getting the client to understand the design within its larger context, as opposed to interpreting a written report. As seen, the case study analysis revealed several important steps that can aid in empowering clients to sustain and grow the design vision. A summary is presented in Vignette 5.0.

Vignette 5.0 Useful Tools to Empower Clients

Empower clients to bring the design in-house and learn to maintain it through:

\section{Client Involvement}

- Involve the client as soon as and as much as possible

- Clients should become active participants in the research, design and development process (design with them)

\section{Salient and Compelling Deliverables}

- Tangible deliverables with clear and actionable recommendations

- Information/data should be represented in a visual way whenever possible

- Real-world stories are compelling and foster empathy 


\section{CHAPTER FIVE}

\section{DISCUSSION}

As the market of goods and services becomes increasingly competitive, many companies are investing more time and money into innovative solutions, hoping to distinguish themselves as industry leaders. As Pine and Gilmore (1999) believed, the opportunity lies in designing for experience. However, in order to achieve the "wow" factor that engenders customer loyalty these experiences must be qualified as rich in meaning and emotion. In addition, as today's society is filled with more and more complexity, designing for experience must also consider how systems of people, things, and places interconnect. Therefore, this study aimed to understand how design consulting firms, entrusted with the task of creating the products and services that ultimately foster these rich user experiences, successfully achieve the latter from a systems perspective.

Consequently, this study set out to address the following research questions: Firstly, what are the components or elements that lead to rich user experience? Next, what are the organizational behaviours and perspectives that must be adopted by design consulting firms, shifting focus from the delivery of products and services to fostering rich user experiences? Lastly, what are the most effective design processes and practices being utilized by experience-centric design consulting firms that ultimately give rise to rich user experiences? Using case study data from seven experience-centric design consulting firms, five patterns emerged: They include a holistic perspective, building an emotional connection, interdisciplinary collaboration, harmonizing touchpoints and client empowerment. 
This chapter will revisit the research questions using the identified patterns as a lens, paying particular attention to the implications for designers working in this growing field. In addition, a Rich Experience Design Diffusion Approach (REDDA) developed through the synthesis of these five patterns is presented. The study's research limitations as well as possibilities for further investigation are discussed next, followed by a concluding section which highlights the criteria that can enable a design firm to develop and design rich user experiences.

\subsection{DISCUSSION OF PATTERNS}

\subsubsection{HOLISTIC PERSPECTIVE}

As the title denotes, a holistic perspective can simply be interpreted as a general propensity towards understanding the "big picture" when designing a product or service. Through the case studies it became clear that a critical ingredient to their success when designing for rich user experience was a consideration of the larger ecosystem of people, contexts, services, and products impacting the design of a singular element. Specifically, participants explained that the way a user experiences a company or brand is a culmination of every interaction had with that organization. As such, it was found that the experience as a whole is impacted positively or negatively depending on the impression of each touchpoint. The latter also supports the findings from the literature review: Wherein experiences result from interactions between people and their environment and an emotional quality of significant impact from one interaction is sufficient to define the overall experience (Arnould and Price 1993; Dewey 1964).

First and foremost, when starting a project, these firms adopted a strategy centered on understanding the broader picture. This holistic thinking radiated throughout the case firms' research and design processes, client interactions, and corporate culture, 
so far as to impact their hiring criteria. They were adept at understanding the product or service on a macro level, viewing everything, whether a human being, a product, a service, or an interface, as a system.

Coupling this finding with the notion of "Systems thinking", presented in the literature review, results in an understanding that each of these elements possesses their own ecosystem and together they overlap and intersect, resulting in countless interactions. Every interaction between and within these systems is an opportunity to create a series of satisfying experiences, culminating in a wholly rich user experience. Therefore, it could be assumed that by shifting their perspective to reflect the complexity of a holistic systems approach, other consulting firms involved in the creation of rich experience may be more effective. Moreover, these findings suggest that firms transitioning towards experience-centered design should look to find new research tools and methods to help their teams fully comprehend the high level of complexity brought about by the limitless number of interactions surrounding their products and services.

The latter implies that even for a product designer, responsible only for a single element within the chain of touchpoints, it is critical to understand the whole picture. Participants warned against designing in a "vacuum" - yet, adopting a holistic perspective and appreciating those inherent complexities, allows critical insights and users' latent emotional needs to be uncovered. Ultimately, helping to create the appropriate solution for the problem at hand, leading to rich user experience.

\subsubsection{BUILDING EMOTIONAL CONNECTION}

In describing successful user experiences, participants noted time and again the importance of building a product or service that was not only functional and usable, but also desirable. The case studies revealed that these firms went to great lengths in building this emotional connection with the user. In order to achieve the latter, they 
employed an array of human centered activities throughout their research and design processes. Including, in-depth ethnographic research, seeking continual user feedback through the use of iterative prototypes and testing, and by involving the user, in varying capacities, through all phases of the project.

They also provided examples of specific research tools they found to be particularly effective in outlining the details surrounding these complex systems - such as experience mapping to acquire a comprehensive understanding of the user-customer journey, as well as building $2 \times 2$ frameworks to understand user groups and the intersections between them. Beyond the level of depth at which they approached a problem, it is important to note the type of research case firms most often utilized when designing for rich user experience. While they regularly conducted interviews and surveys, participants nonetheless attested observational research to be the most effective at uncovering latent needs.

The data analysis revealed that these activities ultimately affected the team by enabling them to empathize with the user and gain a deep understanding of their latent emotional needs. Namely, Macadamian, Kicker Studio, IDEO and Adaptive Path described empathy as a principle driver behind successful experience design. Realworld stories were described as an effective tool to help build this empathy; specifically, case firms often created personas, scenarios, short videos and storyboards to help humanize their research findings. Hence, by embracing the user's perspective designers were able to pinpoint and tackle the issues that would lead the user to experience a positive emotional reaction when engaging with the respective touchpoints.

From these findings a diagram was created (seen in Figure 6.0) to illustrate how the human centered approach affects the project team and the outcomes for the user. 

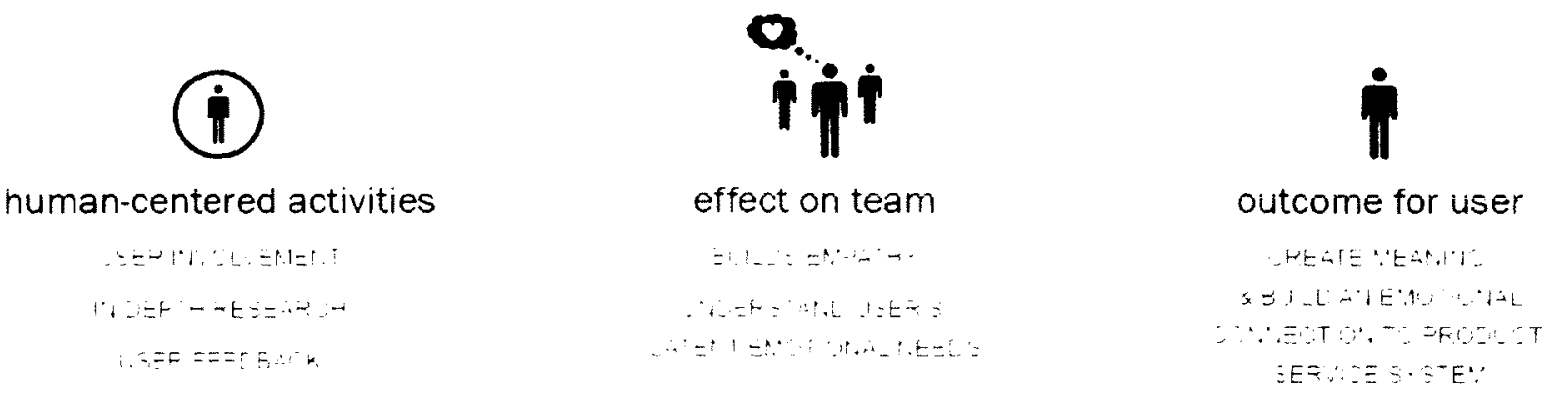

Figure 6.0. Building an Emotional Connection. This figure illustrates how a human centered approach affects the design team and leads to an emotional connection between the user and the company.

In sum, case firms embraced the user's perspective when carrying out the design of any product or service, as well as strived to build empathy across all team and project members. This combined with application of the human-centered research methods outlined above, allowed them to create an emotional connection - a significant component that any product or service must possess if they are to foster rich user experience. While this pattern suggests that consulting firms must still engage in a barrage of human-centered research techniques, it also indicates that they should focus more energy on the emotional impact of their solutions as well as on building empathy across team members.

\subsubsection{INTERDISCIPLINARY COLLABORATION}

It is universally understood that a company is only as good as its people, thus it is not surprising that companies today are so highly focused on hiring the right individuals with the right combination of skills in order to build teams of great chemistry and flow. The case studies revealed that the design firms investigated placed a strong importance on team composition. In particular, participants described their team models as being composed of individuals from varying professional backgrounds, yet who each had a good understanding of other specialties beyond their formal training, often referred to as 
design generalists. In addition, they discussed the way in which team members interacted - not only were teams cross-functional, but they were collocated and whenever possible encouraged to collaborate from beginning to end. Firms stressed the importance of involving each team member in all phases of the design and research, indicating that there is no substitution for first-hand knowledge. This type of continual consideration and dedication to team structure demonstrated a common organizational behavior exhibited by the case firms.

Perhaps the most interesting finding from the research was the emergence of the design generalist as a key player in team make-up, particularly when designing for rich user experience. Although no definitive definitions of the term were provided from the case studies, through the interviews and document analysis it is surmised that a design generalist has their central domain knowledge (formal training) bolstered by satellite skills from other design and research disciplines, affording them the capacity to assume different roles through and across projects. It is important to note that the study focused on design firms and although a few of the consulting firms had in-house marketing, and engineering departments, the emphasis is placed on the design disciplines and research capacities. Consequently, the designers and researchers may hold degrees from other domains, including engineering, sociology, anthropology, or marketing but their professional experience and job titles are as designer and/or researcher.

As previously noted in the first identified pattern, experience design inherently requires big picture thinking and seamlessly integrated solutions across all touchpoints. As such, it could be deduced that the emphasis case firms placed on hiring design generalists may have been due to the holistic perspective necessary when designing for rich experience, as individuals capable of assuming multiple roles in or across projects are innately more open-minded and capable of understanding the bigger picture (Choi and Pak 2006). In all likelihood, teams who habitually engage in cross-pollinating and 
sharing ideas and perspectives automatically open themselves up to finding more insightful design opportunities.

The type of collaboration exhibited by these firms is significant as it differs from the conventional multidisciplinary team model in several key dimensions.

Multidisciplinary cooperation, a prevalent team model in industry, involves individuals from respective disciplines coming together at strategic points during a project, where each individual acts as the advocate or guru of their particular discipline (Blevis and Stolterman 2008; Choi and Pak 2006). As such, they are responsible for all project tasks specific to their functional domain. Generally, these teams will come together in occasional meetings and subsequently return to their individual workspaces, siloed from other perspectives. This type of team structure leads to numerous hand-offs between functional groups, something to be adamantly avoided as far as participants were concerned.

From the interviews it could be postulated that every hand-off acts as a filter, resulting in valuable insights lost at each stage throughout a project. An analogy could be made to the childhood game of "telephone": Where players whisper a word/phrase from one person to the next, and inevitably, at the end of the chain the original phrase has been distorted beyond recognition. Only instead of a word or simple phrase, we are dealing with complex sticky systems involving many stakeholders and scenarios.

An interpretation of these results assumes that the reports and presentations used during these hand-offs only impart explicit knowledge (knowledge that can be readily transferred to others through media, writing, manuals, etc.) to the individual on the receiving end. This exacerbates the issue, as critical insights discovered throughout the research phase often fall through the cracks and are unintentionally omitted from reports (Merholz et al. 2008). In essence, there is so much more to be understood in these complex webs of products, services, contexts, and people than could possibly be 
communicated via reports and presentations. As a result, these types of team models can result in products and services designed in isolation, without consideration of the entire system. The diagram depicted in Figure 7.0 attempts to demonstrate the latter and what can happen to elements of a system designed through multidisciplinary collaboration.

In contrast, interdisciplinary collaboration, as previously defined, was a seemingly crucial element of case firms' organizational behaviors. Moreover, it helps to minimize the silo effect and hand-offs, which they stressed, leads to a loss of insights. Thus, the findings suggest that by having individuals who cross over functional lines, taking on multiple roles within a project, even if only in a small capacity, allows all team members to understand what cannot be readily understood through reports and presentations. It is the concept of tacit knowledge transfer (knowledge that can't readily be explained in words): Wherein, team members who conduct some of the research and some of the design in essence remove functional barriers and carry the insights from each phase, forward to the next. Figure 8.0 depicts interdisciplinary collaboration and the resulting outcome on a product ecosystem.

Therefore, through the empirical study it could be postulated that team involvement in all phases, as well as hiring design generalists ensures continuity of insights and knowledge throughout each phase of the design process. This also seemed to lead to a more comprehensive understanding of the "whole" and cultivated the ideal conditions for designing products and services capable of eliciting rich user experiences. 


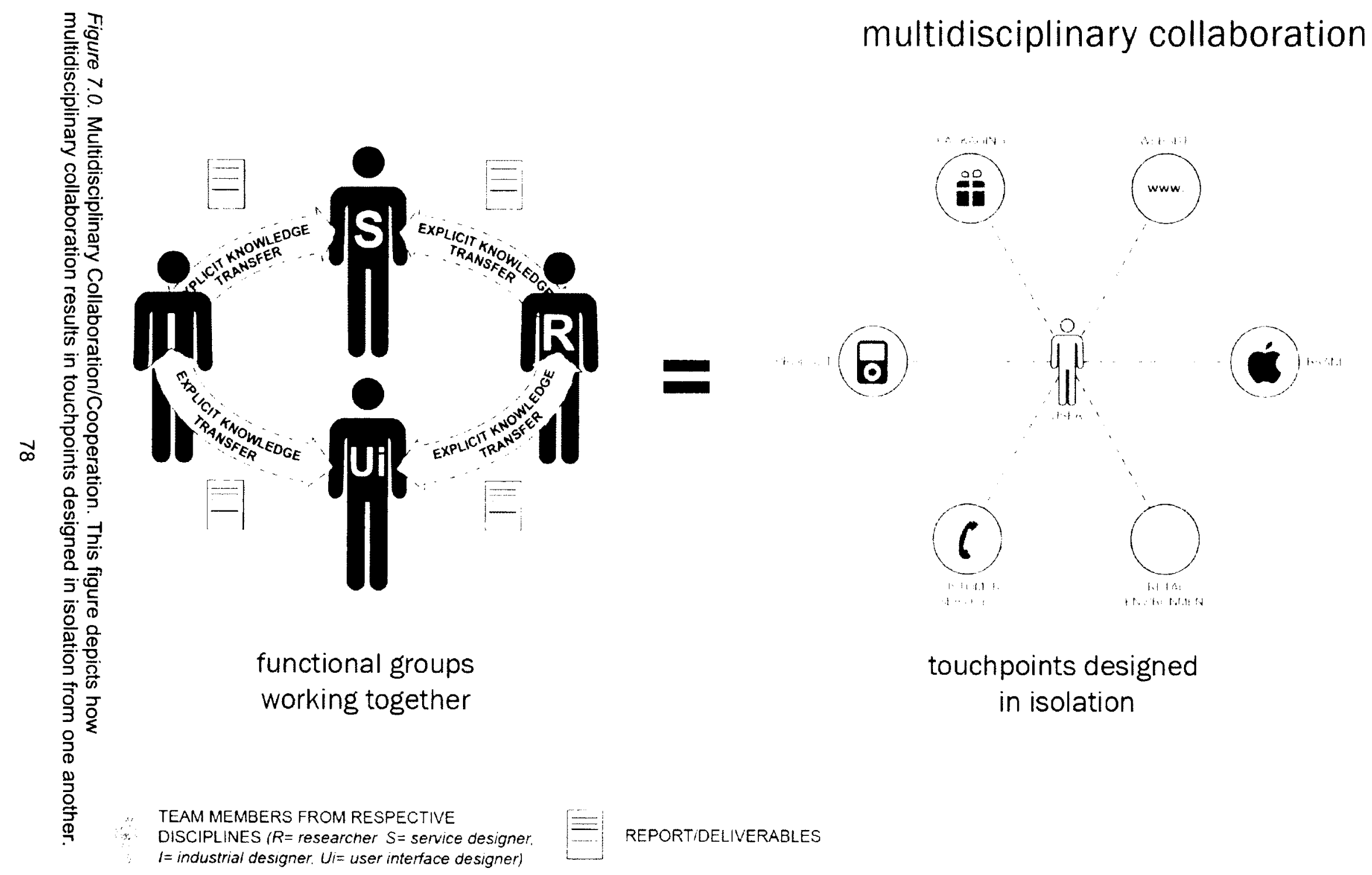



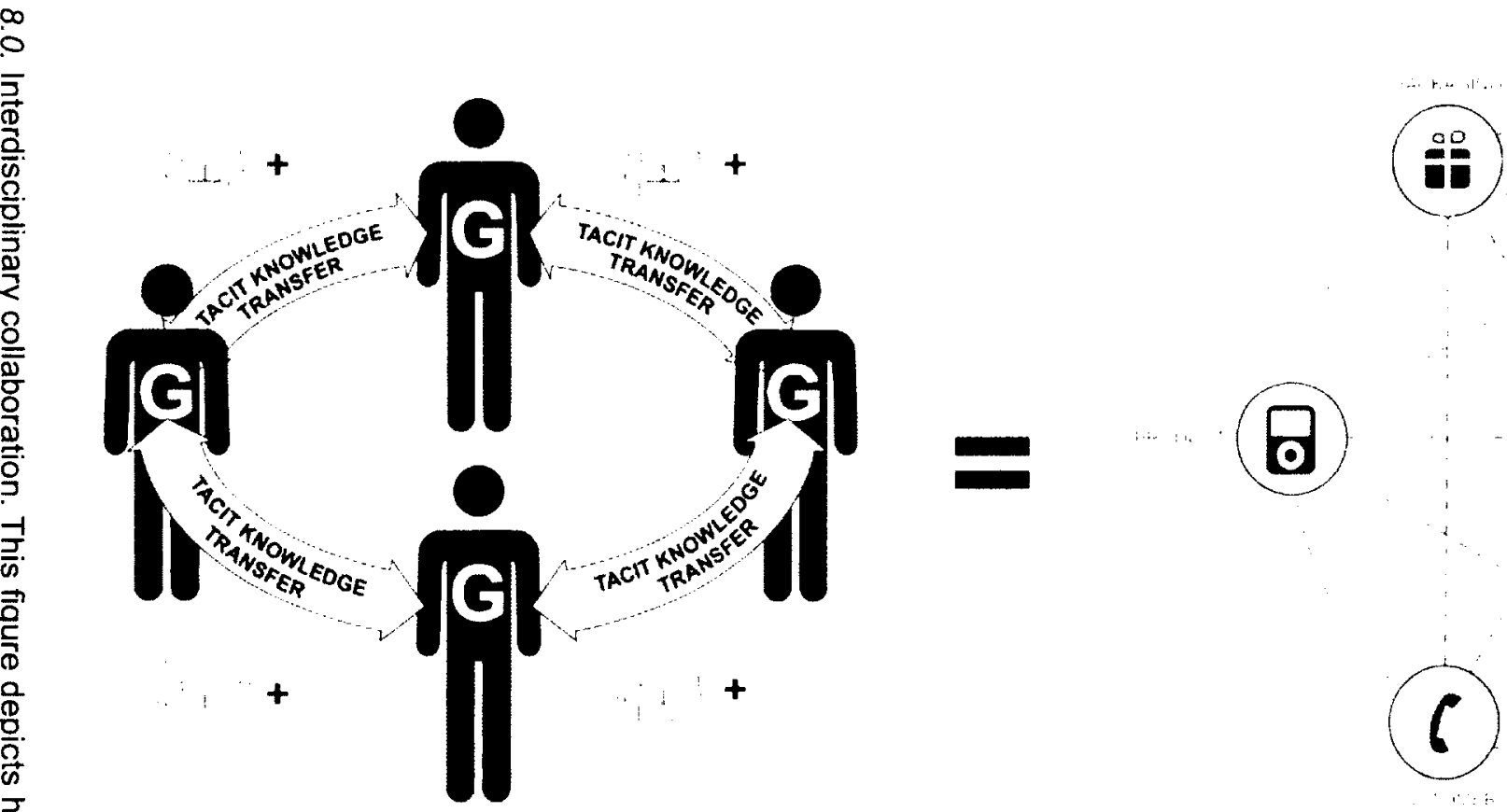

interdisciplinary collaboration

cross-pollinating

design generalists

touchpoints designed

in an ecosystem 


\subsubsection{HARMONIZING TOUCHPOINTS}

Further to the idea of interdisciplinary collaboration was the importance of having a team with a broad-reaching skill set and thus the capacity to deliver an integrated experience across multiple touchpoints. Interestingly, while the notion of harmonizing touchpoints was expressed by nearly all participants, Ziba Design and Kicker Studio were two firms which persisted on the topic. It became evident through the case study analysis that the design firms recognized and strived to achieve a consistent user experience throughout the user-customer journey. In order to achieve this consistency they spoke of designing and carefully executing each touchpoint, staring from the smallest detail in the product packaging to employee-customer interactions. This closely ties the pattern of harmonizing touchpoints with that of a holistic perspective, reiterating the importance placed on a firm's ability to design for the micro while understanding the macro.

The concept of harmonizing touchpoints mirrors discussions from the services sector presented in the literature (Zomerdijk and Voss 2010; Pine and Gilmore 2011; Shedroff 2009). Nonetheless, these findings bring new insights and techniques specific to designers and are of particular use to those unable to design across multiple touchpoints either due to contractual limitations or separate functional groups, however ill advised. In order to overcome these challenges many of the case firms presented best practices to implement that can be used internally and also to extend externally to the client.

Specifically, these firms created overarching design principles or themes that they used to align everyone on the team to a common design vision. Participants described similar definitions/concepts, referring to a "North Star" while others referred to design principles or guidelines. Through the interviews it is possible to interpret a design 
vision as a set of rules describing the emotive elements to be elicited through a set of interactions.

Similarly, other participants described them as a set of attributes or strategy that denotes both company personality and a review of how people will exchange with the touchpoints. This idea is of particular interest to designers and managers in industry, serving as a practical tool that they can use when trying to impact the system on a larger scale, even if only immediately involved with one touchpoint. This concept of presenting the user a harmonized experience seemed to be a component leading to rich experience, as did building an emotional connection.

\subsubsection{CLIENT EMPOWERMENT}

Extending this notion also means imparting a design vision to clients so they are able to sustain and grow the vision once the consulting firm has moved on. The concept of client empowerment stood out as perhaps the most unique and novel among all of the design patterns identified. Participants strongly believed that their work as designers was only truly complete when they could successfully empower their clients. They expressed that, in the end, even great ideas fail with poor execution and lack of maintenance. Moreover, the case firms ultimately felt that their clients have to take ownership of the design vision and learn how to apply it properly, if rich user experiences are to be sustained.

The latter denotes a shift in the traditional designer-client paradigm, whereby clients have moved from being idle receivers of deliverables to active participants in the research, design and development process. This approach is in contrast to the conventional "curtain" model where designers would unveil the design at various project milestones, using presentations and research reports as validation for design decisions made. Rather, it follows the new trend towards co-design. However the commonly 
understood definition of co-design is one where users become actively involved in the design process, whereas client empowerment shifts it to also including the client. The latter suggests that designers are to become the facilitators and enablers of the design vision - the evangelists. To some extent this involves assessing a client's readiness to accept their new roles as participants in the design and research phase and subsequently, as the design caretaker. In one of the cases, the firm under investigation even went so far as to turn away clients whom they didn't feel had the organizational maturity and openness to take on such responsibility. While turning away clients is perhaps extreme and not always feasible for many consulting firms, it surely emphasizes the importance some of these firms placed on client empowerment.

It is important to note that only three of the seven firms expressed the client's role as paramount in considering when designing for rich experience, and was central to their strategy when tackling any problem. Nonetheless, client empowerment was singled out as a pattern because of its novelty and emphasis. In particular, IDEO, Adaptive Path and Ziba Design adamantly expressed these ideas. This may have been due to firm size and available resources, as well as their time in industry.

Of all the patterns, client empowerment may be the most difficult to adopt, as designers often resist changes that imply a loss of control. However, a clarification must be made in that this concept is not meant to suggest that clients become designers, familiar with the ins and outs of all processes. Rather, it suggests that consulting firms supply the knowledge and essential tools clients require in order to apply the design within other contexts, and ensure that their own teams are appropriately implementing the vision.

In an attempt to interpret the data, a diagram was created to visually represent this relationship using the metaphor of a seed that is nurtured and blossoms into a flower. Figure 9.0 depicts this metaphor as the designer planting a seed or "design" and 
uses tools and techniques to actualize the design (or seed into a flower). Along the way, he involves the client as an active participant in the design and development process and subsequently aids during the implementation phase. Meanwhile the client is heavily involved in all decisions made. Eventually, when the designer moves on, the client has the tools and knowledge needed to sustain the design (or flower) allowing it to live on and even grow. As a caveat, should the client wish to grow a brand new species of flower (or new design) he must enlist the designer's aid once again.

If designers are to accept this new role, they must learn the tools that will enable their clients to take ownership of the design once they are gone. Decidedly, participants recognized that deliverables still remain a crucial bridge between designers and clients. However, they were specific in the types of deliverables to be used - they must be actionable and compelling and should also involve the use of stories and information visualization. Similarly, one participant emphasized that as work in this field becomes more and more abstract and less tangible, more people are needed who can communicate these concepts to others in a compelling way that transcends functional language barriers. In discussing the benefit of information visualization one participant stated: "It was a tantalizing way to represent information that can otherwise be overlooked or forgotten."

Training was also a significant component in imparting the knowledge clients needed. However, no matter how strong the deliverable or the workshop they cannot replace a client's active participation in the design and development phases. 


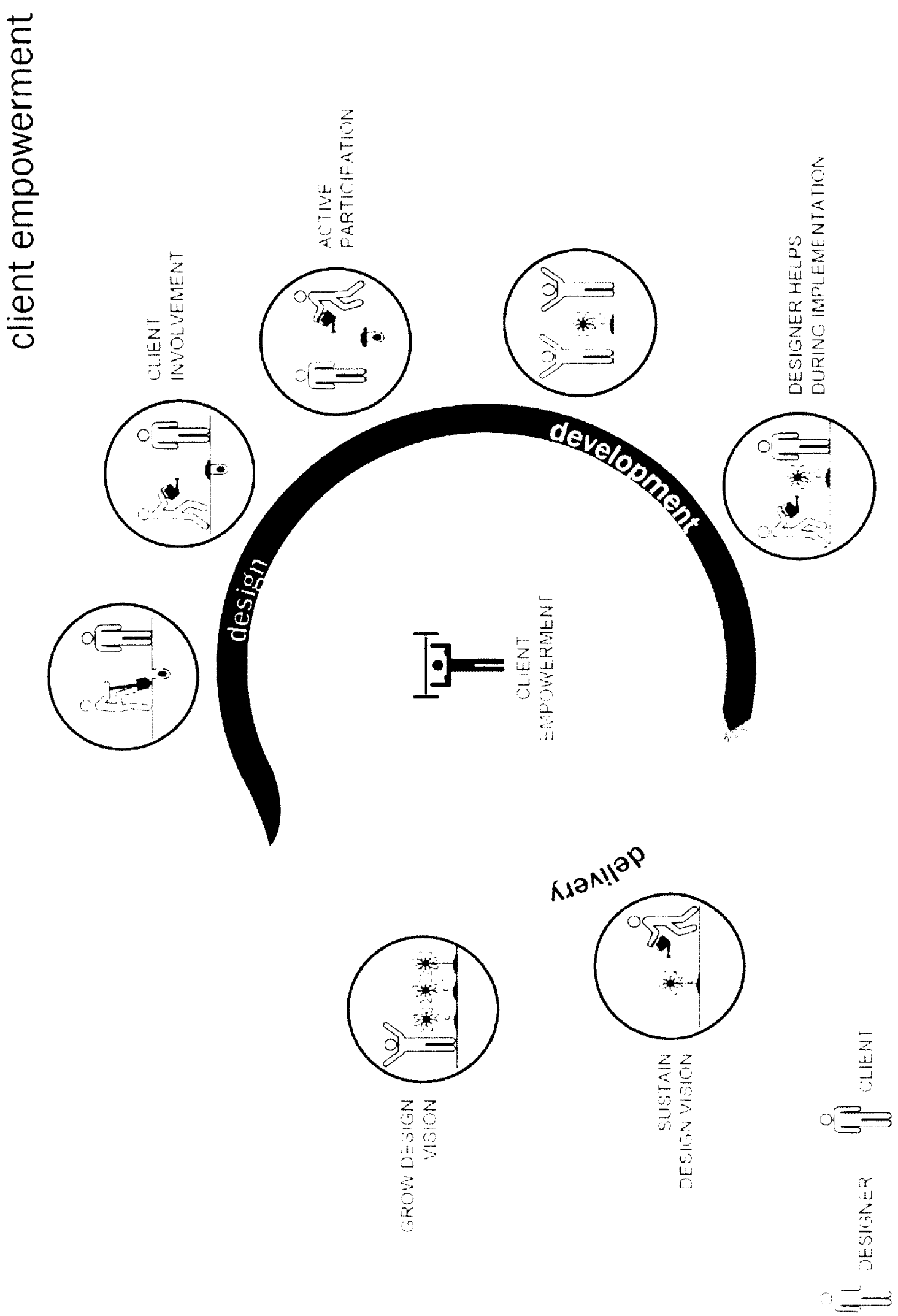

Figure 9.0. Client empowerment. Using the metaphor of a seed, this figure depicts how a designer can enable client empowerment through the various phases of a project. 
In effect, even a firm that has successfully embraced a holistic perspective, applied a human centered approach in building an emotional connection with the user, and is capable of harmonizing touchpoints, is still dependent on the success their clients. This pattern demarks new behaviours between client and designers and as a result has the potential to change every interaction between these previously segregated groups; this may denote a higher frequency of contact, as well as new mediums for mutually sharing ideas. This pattern helps to round out the other four patterns and complete the progression towards designing for rich user experience.

\subsection{IMPLICATIONS}

As shown, the five design patterns identified through the case study analysis are heavily intertwined; henceforth, it could be assumed that they work most effectively when applied in conjunction with one another and that together they present significant implications for the design community at large. Consequently, it is possible to connect and organize these patterns into an actionable design approach for managers and designers alike. Figure 10.0 depicts a diagram that synthesizes the patterns into a Rich Experience Design Diffusion Approach (REDDA). This section will first focus on explaining the connections and actions brought about through REDDA, followed by a discussion of the general implications of this research for the design field.

First and foremost, a design consulting firm, prior to beginning any project, must embrace a holistic perspective. This perspective will then support them in understanding the relationships and impact of each touchpoint along the user-customer journey, as well as the complexity encircling the users and stakeholders and spreading through all other business, research and design activities. Firms must then employ a process that is rich in human centered activities leading to an in-depth understanding of the user's emotional 
latent needs in order to design for them. Interestingly, if we revisit two previous statements when defining rich experience:

1. Rich experiences are generated when a person forms an emotional connection to an interaction with an element of their environment.

2. Rich experiences are created as the result of a series of smaller satisfying experiences, unified within a product's ecosystem.

We see that rich experience is not possible without positive emotional connection. Furthermore, the second statement supports the third pattern related to harmonizing touchpoints. As such, both could be considered components of rich experience. Subsequently, firms must focus their attention on delivering a consistent experience across all touchpoints - when one component of the system fails the entire experience is broken. Once again, maintaining a holistic perspective throughout all processes will help firms in understanding the "big picture" and how all of the touchpoints fit together. Moreover, it will aid them in developing the overarching design principles and guidelines that provide the attributes of the desired experience at each touchpoint. However, these three elements - a holistic perspective, emotional connection, and harmonizing touchpoints - alone are still insufficient for delivering the kind of experience that results in the "wow" factor and true staying power of a product or service. There are two organizational competencies, one internal and the other external that allow those other three patterns to thrive: Interdisciplinary collaboration and client empowerment. The former denotes an internal behavior of team structure that allows for knowledge and insight continuity across all phases, as well as a better opportunity to discover the insights needed that lead to fulfilling designs. Client empowerment denotes a 
relationship between the firm and their client that must be fostered in order to allow the design to flourish beyond the walls of consulting firm.

Nelson and Stolterman (2003) raised an important issue on the topic of systems and the emergent field of experience design: Noting that the systems approach, inherent in the first pattern of holistic thinking, is paradoxical in academia. They explain that domain specialization is characteristically narrow-minded, as specialization necessitates deep immersion in one area, resulting in a diluted or absent understanding of the other fields overlapping it. However, systems require a more general understanding of all domains, yet to date in academia, attempts to gain disciplinary knowledge from a more inclusive perspective have been viewed as superficial. Further emphasizing the importance for professionals involved in systems design to gain the ability to move between macro and micro perspectives, which was a proficiency exhibited by case firms through employment of design generalists.

If this new breed of designers is to resolve these paradoxical worldviews, how does one become a design generalist? While the answers to this question move beyond the scope of this study, these findings nevertheless have significant implications for all manner of designers, whether new to the field or well established. The advent of the design generalist supposes that future designers will need to work on broadening their skill sets and implies that design educators may require new teaching methods in order to foster a wider skill base in their students. Although this research was conducted in the context of design and research firms, the concept of domain generalists could be extended to other disciplines such as engineering and marketing. Moreover, it may even suggest that companies should invest in training and shadowing programs for their employees. The latter could also aid in breaking down functional language barriers. 


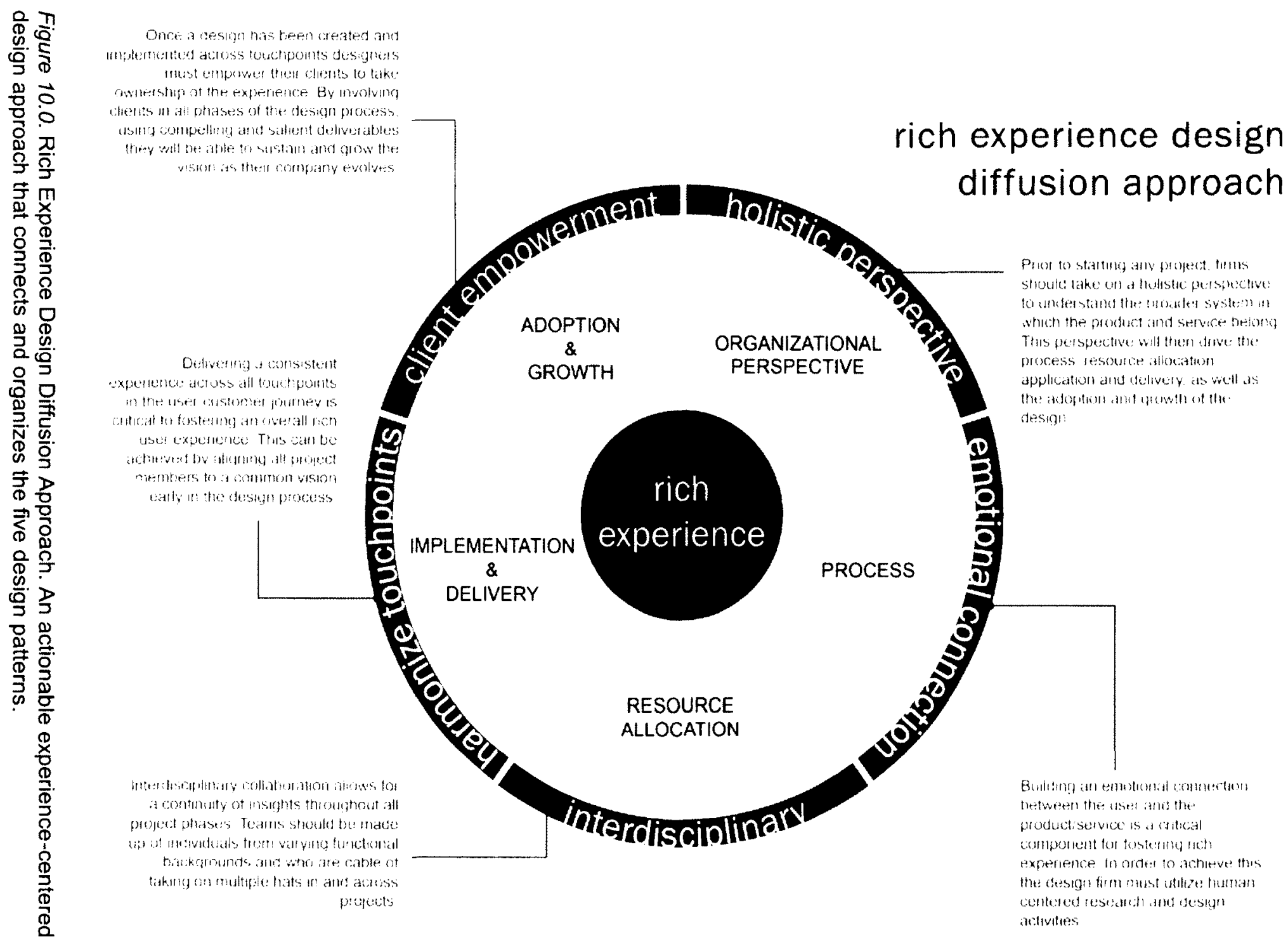


Furthermore, through the case study analysis it appeared that firms which embraced a holistic perspective worked to overcome these barriers and find compelling means for communicating complexity: As "our dominant mode of communication, the linear format of text, falls short when used without the corroboration of other means of representing complex dynamic entities" (Nelson and Stolterman 2003, 87). Thus, how can a complex system be accurately represented through the deliverables they create? The case firms revealed several tools, such as storyboards, scenarios, videos, and experience maps, however, these tools were only examined superficially by the researcher. In fact, Ziba Design, Method, and Adaptive Path were three firms that repeatedly mentioned storytelling as critical in understanding and translating the complexity surrounding users, and imparting contextual information to other parties. It became clear that two concepts "Stories" and "Empathy" were reiterated throughout the interviews and document analysis, however based on the research problem, the choice to amalgamate and disperse them throughout the patterns rather than separating them out as unique patterns seemed more appropriate.

While the findings from this study offer practical tools for design consulting firms transitioning to an experience-centered culture, however, this evolution requires a significant level of organizational change. Especially for those firms which have only recently embraced the power of a design culture. As such a firm wishing to employ these principles or REDDA, should first look within their organization and assess readiness among their employees. 


\subsection{LIMITATIONS AND FUTURE RESEARCH}

\subsubsection{LIMITATIONS}

The limitations surrounding this study resulted both from the sampling technique utilized by the researcher, as well as the time and resource restrictions placed on the study. The resulting implications are discussed below.

Firstly, employing a snowball sampling technique resulted in a disproportionate amount of participants from a User Experience (or $\mathrm{HCl}$ ) Design background. Namely, Method, Macadamian, Kicker Studio, Akendi, and Adaptive Path all started out as UX design or software innovation firms. While several of them have branched out to include service and communication design, many of their principles are based on traditionally UX doctrines. However, all participants understood the context of the study prior to partaking and all demonstrated a critical appreciation for experience design from a systems approach. Nonetheless, future studies in this area should aim to achieve a broader sample of case study firms, from every avenue of design.

Moreover, beyond varying in type of business offering, consulting firms also varied in size and available resources. Specifically, three of the firms did not have inhouse engineering and marketing departments and as a result their interpretation of interdisciplinary collaboration may have been on a smaller scale and based more on diversity within the design and research team. As a result of the varying team structures, these firms also had slightly different understandings of communication tools.

In addition, participants themselves also held different job titles from one another and as such had differing perspectives on experience. For example, one participant started their career as a researcher, and therefore placed much greater importance on the impact of research over design. Others had a broader view of the whole situation and some had more in-depth, tailored knowledge and experience in certain areas. This 
diversity in participant job role and background was purposefully chosen so as to achieve a broad sample with varying perspectives, however, as a result the generalizability of the results remains tentative. To help mitigate some of these disparities a rigorous document analysis was conducted on each firm in order to support the findings from the interviews, as well as to fill in the gaps - where one participant may not have had views that accurately represented the entire capacity of the firm under investigation.

Lastly, and perhaps most significantly, while the case study analysis presented valuable results for designers and managers involved in rich user experience design, due to time and resource restrictions a study to validate these findings was not conducted. As a result, these findings are speculative and are not intended to be prescriptive. Rather, they act as a critical stepping-stone in the pursuit towards defining and demystifying this new design space.

\subsubsection{FUTURE RESEARCH}

As previously mentioned, while this study has made significant strides in developing actionable business, research, and design processes, more should be done to substantiate the identified patterns as a mainstay in the Experience Design domain. After discussing the research limitations, an advantageous next step would validate these patterns through a design exercise: Conceivably, conducting a case study of an existing company while employing the five design patterns to redesign it, or given the opportunity, infiltrating a design team and applying these techniques in tackling a problem to understand their effectiveness.

What is more, this study utilized grounded theory and as a result approached the area of research broadly. It began simply with a keen interest in learning how to create products and services that are continually enthused over and widely praised. 
Consecutively, three general research questions surrounding the creation of rich user experience were developed. However, as a result of employing this type of bottom-up research approach, the set of design patterns uncovered only skim the surface and due to time limitations do not address the specific "how-to's" behind each one, as participants only provided a handful of examples during the interviews. This is primarily attributable to the fact that the interview protocol was tailored more towards understanding the general approaches and behaviours case firms regularly utilized when designing for experience. As such, future research should delve more deeply into each pattern and investigate the explicit tools and artifacts firms use when attempting to build an emotionally charged, harmonized experience for user's and customers.

Furthermore, future research may also look to build a robust taxonomy of systems in order to solidify the patterns identified here. These types of systems, whether product based, service based, retail oriented, or based on internal organizational structure could be used as a lens through which to apply the patterns and develop new principles spanning across the various system categories. Perhaps even building experience design toolkits specific to system type.

Although this study examined the components of rich experience and found them to surround emotional connection and harmonized touchpoints, these were discovered from the consultant's perspective. Therefore, an analogous study could be conducted, only from the user's viewpoint. This could involve interviewing and observing users and stakeholders to uncover the elements that they find either make a good or bad experience in regards to the products and services they use. 


\subsection{CONCLUSION}

In discussing the implications of these finding on the larger field of design, the analysis revealed an emergent design space where designers must shift their perspective from the isolated product or service towards a system of interconnected products, places and people. The latter was particularly evident when reporting on employing a holistic perspective throughout the design approach. Moreover, designing for experience denotes a new competitive market. These patterns can aid in redirecting organizational focus and help companies to recognize that every touchpoint impacts the greater experience. What is more, it is evident through the growing focus on creating interdisciplinary teams that previously segregated design and research roles are fusing. This transcends the traditional disciplinary casts towards building a new breed of designers: Design generalists. Similarly, the shift in the existing client-designer paradigm towards participatory design ascertains that designers will play a greater and greater role as facilitator, communicator and enabler for their clients. More specifically, through the grounded theory approach, utilizing inductive reasoning, it can be hypothesized that,

A design consulting firm can foster rich user experience through the services and products they design if,

H1: they employ a holistic system's perspective in understanding the complexities resulting from the web of interconnected people, places, and things.

H2: they employ a human-centered process to all research and design activities in order to build team empathy, allowing them to uncover the user's emotional latent needs. 
H3: they harmonize the interactions between a user and a company's touchpoints, enabling the user to feel a positive emotional connection at each stage throughout the user-customer journey.

H4: they utilize interdisciplinary collaboration to obtain varying perspectives and maintain insight continuity through all phases of the research, design, and development processes.

H5: they empower their clients to take ownership of the design vision in order to have them maintain and grow it, once the firm has moved on.

In conclusion, by utilizing these patterns and incorporating the components, behaviours, methods and perspectives presented throughout, a consulting firm should be able to successfully foster rich user experience through the products and services they create. In turn, this would ultimately enable their design to live and thrive in the competitive battleground of the experience economy. 


\section{REFERENCES}

Abrahams, Roger D. 1986. Ordinary and extraordinary experience. In The anthropology of experience, eds. Victor W. Turner, and Edward M. Bruner, 45-72. Urbana, IL: University of Illinois Press.

Adaptive Path. 2011a. In-house training. http://adaptivepath.com/work/services/inhouse-training (accessed February 28, 2012).

_. 2011b. Services. http://adaptivepath.com/work/services (accessed February $28,2012)$.

- 2011c. Work. http://adaptivepath.com/work (accessed February 28, 2012).

Risdon, Chris. 2011. The anatomy of an experience map. Adaptive Path blog, entry posted November 20. http://adaptivepath.com/ideas/the-anatomy-of-anexperience-map (accessed February 28, 2012).

Akendi. 2012b. Our services. http://www.akendi.ca/customer-user-experiencedesign/customer-user-experience-research-design-services.php (accessed February 28, 2012).

Altheide, David. 1996. Qualitative media analysis: Vol. 38. Qualitative research methods. Thousand Oaks, CA: Sage Publications.

Arnould, Eric. J., and Linda L. Price. 1993. River magic: Extraordinary experience and the extended service encounter. Journal of Consumer Research 20, no.1: 24-45.

Auerbach, Carl F., and Louise B. Silverstein. 2003. Qualitative data: An introduction to coding and analysis. New York, NY: New York University Press. 
Backett, Paul. 2011. Rethinking design education. Ziba Design. Whitepaper. http://www.ziba.com/perspectives/topic/beautifulexperiences/\#/perspectives/articl e/rethinking-design-education/ (accessed February 28, 2012).

Bak, Per. 1996. How nature works: The science of self-organized criticality. New York, NY: Springer-Verlag.

Bitner, Mary J. 1992. Servicescapes: The impact of physical surroundings on customers and employees. Journal of Marketing 56 (April): 57-71.

Blevis, Eli, and Erik Stolterman. 2008. The confluence of interaction design and design: From disciplinary to transdisciplinary perspectives. Proceedings of DRS2008: Design Research Society Biennial conference, 1-12, in Sheffield, UK.

Brown, Tim. 2009. Change by design: How design thinking transforms organizations and inspires innovation. New York, NY: Harper Business.

Brunner, Robert, and Stewart Emery. 2009. Do you matter: How great design will make people love your company. Upper Saddle River, NJ: Pearson Education.

Bui, Yvonne N. 2009. How to write a master's thesis. Thousand Oaks, CA: Sage Publications.

Choi, Bernard C. K., and Anita W.P. Pak. 2006. Multidisciplinarity, interdisciplinarity and transdisciplinarity in health research, services, education and policy: Definitions, objectives, and evidence of effectiveness. Clinical Investigative Medicine 29, no. 6: 351-364.

Conklin, Jeff. 2006. Wicked problems and social complexity. In Dialogue mapping: Building shared understanding of wicked problems, 3-40. West Sussex, England: John Wiley \& Sons.

Csikszentmihalyi, Mihaly, and Isabella S. Csikszentmihalyi, eds. 1988. Optimal experience: Psychological studies of flow in consciousness. Cambridge: Cambridge University Press. 
Csikszentmihalyi, Mihaly, and Eugene Rochberg-Halton. 1981. The meaning of things: Domestic symbols of the self. Cambridge: Cambridge University Press.

Dewey, John. 2005. Art as Experience. New York, NY: Perigee. (Orig. pub. 1934.) Dubberly, Hugh, and Shelly Evenson. 2008. The experience cycle. Interactions Magazine, May 01. http://www.dubberly.com/articles/interactions-the-experiencecycle.html (accessed March 01, 2012).

Dust, Fred, and llya Prokopoff. 2009. Designing systems at scale. Rotman Magazine, Winter.

Edgall, Stephen, Kevin Hetherington, and Alan Warde, eds. 1997. Consumption matters: The production and experience of consumption. Oxford: Blackwell.

Farnham, Kevin. n.d. Holistic brand experiences. Method. SWF file. http://method.com/\#/thoughts/people/detail/people/holistic-brandexperiences (accessed February 28, 2012).

Forlizzi, Jodi L. 1997. Designing for experience: An approach to human-centered design. Master's thesis, Carnegie Mellon University.

- 2008. The product ecology: Understanding social product use and supporting design culture. International Journal of Design 2, no.1: 10-19.

Forlizzi, Jodi L., and Shannon Ford. 2000. The building blocks of experience: An early framework for interaction designers. Paper presented at the 3rd conference on Designing Interactive Systems: Processes, Practices, Methods, and Techniques, in Brooklyn, New York.

Garret, Jesse J. 2003. The elements of user experience: User-centered design for the web. Berkely: New Riders Press. 
Hassenzahl, Mark, Sarah Diefenbach, and Anja Goritz. 2010. Needs, affect, and interactive products: Facets of user experience. Interacting with Computers 22: 353-362.

Hately, Matthew. 2011. Google+Motorola=Apple? Puhleeeze. Macadamian blog, entry posted August 18. http://www.macadamian.com/blog/post/google_motorola_apple_puhleeeze/ (accessed February 28, 2012).

IDEO. 2009. Long-haul travel experience for Air New Zealand.

http://www.ideo.com/work/long-haul-travel-experience/ (accessed February 28, 2012).

2010. Designing the ideal home for "Wounded Warriors" for Clark Realty. http://www.ideo.com/work/designing-the-ideal-home-for-wounded-warriors/ (accessed February 28, 2012).

- 2012. Careers. http://www.ideo.com/contact\#careers (accessed February 28 , 2012).

Khalid, H. M., and M. G. Helander. 2006. Customer emotional needs in product design. Concurrent Engineering: Research and Applications 14, no.3: 197-206.

Macadamian. 2012. Training. http://www.macadamian.com/services/training/ (accessed February 28, 2012).

Margolin, Victor. 1997. Getting to know the user. Design Studies 18, no.3: p227.

McCarthy, John, and Peter C. Wright. 2004. Technology as Experience. Cambridge, USA: MIT Press.

Meredith, Jack. 1998. Building operations management theory through case and field research. Journal of Operations Management 16, no.4: 441-454. 
Merholz, Peter, Brandon Schauer, David Verba, and Todd Wilkens. 2008. Subject to change: Creating great products and services for an uncertain world. Sebastopol, CA: O'Reilly Media.

Murdock, Robert. n.d. How to be a successful designer. Method. SWF file. http://method.com/\#/thoughts/people/detail/people/how-to-be-a-successfuldesigner (accessed February 28, 2012).

Mutlu, Bilge D. 2004. The chaotic nature of human experience: Insights on the subject matter of design towards sstablishing a science of design. Master's thesis, Carnegie Mellon University.

Neilson, Jakob. 2005. Retrieved from http://www.useit.com/papers/heuristic/heuristic_list.html (accessed January 20, 2012).

Nelson, Harold G., and Erik Stolterman. 2003. The design way: Intentional change in an unpredictable world. Englewood Cliffs, NJ: Educational Technology Publications. Newbery, Patrick. n.d.(a) Climbing out of the silo. Method. SWF file. http://method.com/\#/thoughts/all/detail/people/climbing-out-of-thesilo (accessed February 28, 2012).

Norman, Don A. 2004. Emotional design: Why we love (or hate) everyday things. New York, NY: Basic Books.

Norman, Don A. 2002. The design of everyday things. New York, NY: Basic Books. (Orig. pub. 1988).

O'Reilly, Terry. 2012. "A new BRIC in the wall." Under the Influence. Podcast audio program. CBC Radio, January 7, http://www.cbc.ca/undertheinfluence/ (accessed January 20, 2012). 
Pine, Joseph B., and James H. Gilmore. 1998. Welcome to the experience economy. Harvard Business Revew 76: 97-105.

-1999. The experience economy: Work is theatre and every business a stage. Boston, MA: Harvard Business Review Press.

2011. The experience economy: Work is theatre and every business a stage. $2^{\text {nd }}$ ed. Boston, MA: Harvard Business Review Press.

Popovic, Vesna. 2002. Activity and designing pleasurable interaction with everyday artifacts. In Pleasure with products: Beyond Usability, eds. William. S. Green, and Patrick. W. Jordan, 365-376. London: Taylor and Francis.

Pourdehnad, John, Erica R. Wexler, and Dennis V. Wilson. 2011. System and design thinking: A conceptual framework for their integration. Paper presented at the $55^{\text {th }}$ annual conference on International Society for the Systems Sciences, July 17-19, in Hull, UK.

Pullman, Madeleine E., and Michael A. Gross. 2004. Ability of experience design elements to elicit emotions and loyalty behaviours. Decision Sciences 35, no.3: $551-578$

Rodriguez, Diego. 2006. "Products and their Ecosystems: Understanding the power of context in product innovation." Core77 Design 2.0: Discussions on Strategy and Innovation. June 6. Mp3

file. http://www.core77.com/development/design2.0/src/Core77_peter_rojas. mp3 (accessed February 28, 2012).

Sanders, Elizabeth B.-N. 2002. Scaffolds for experiencing in the new design space. In Information Design Institute for the Information Design Japan. IID. J, Graphic-Sha Publishing Co., Ltd. 
-2001. Virtuosos of the experience domain. Paper presented at the 2001 IDSA Education Conference.

Schmitt, Bernd H. 1999. Experiential marketing: How to get customers to sense, feel, think, act, and relate to your company and brands. New York, NY: The Free Press.

Shedroff, Nathan. 2009. Experience design 1.1. San Francisco, CA: Experience Design Books.

Sheng, Chieh-Wen. and Ming-Chia Chen. 2011. A study of experience expectations of museum visitors. Tourism Management 33, no.1: 53-60.

Sommer, Barbara, and Robert Sommer. 1997. A practical guide to behavioral research: Tools and techniques. $4^{\text {th }}$ ed. New York, NY: Oxford University Press.

Sommers, Montrose, and James G. Barnes, eds. 2004. Marketing. $10^{\text {th }}$ ed. Toronto: McGraw-Hill Ryerson Limited.

Stuart, lan F. 2006. Designing and executing memorable service experiences: Lights, camera, experiment, integrate, action! Business Horizons 49: 149-159.

Thorne, Frederick C. 1963. The clinical use of peak and nadir experience reports. Journal of Clinical Psychology 19, no.2: 248-250.

van Gelderen, Tedde. 2010. Experience lifecycle strategies: Keys to creating an experience-oriented culture. Akendi. Whitepaper. http://www.akendi.ca/end-toend-experience-design-process/akendi-experience-design-process.php (accessed February 28, 2012).

Veloutsou, Cleopatra, and Luiz Moutinho. 2009. Brand relationships through brand reputation and brand tribalism. Journal of Business Research 62: 314-322.

Vossoughi, Sohrab. 2010. Designing beautiful experiences. Ziba Design. Whitepaper. 
www.ziba.com/perspectives/topic/beautifulexperiences/\#/perspectives/article/ designing-beautiful-experiences/ (accessed February 28, 2012).

Walls, Andrew R., Fevzi Okumus, Youcheng Wang and David Joon-Yuk Kwun. 2011. An epistemological view of consumer experiences. International Journal of Hospitality Management 30: 10-21.

Yin, Robert K. 1994. Case study research: Design and methods. $2^{\text {nd }}$ ed. Thousand Oaks, CA: Sage Publications.

Zhou, Feng, Qianli Xu, and Roger J. Jiao. 2011. Fundamentals of product ecosystem design for user experience. Research in Engineering Design 22: 43-61.

Ziba Design. n.d. About. http://www.ziba.com/perspectives/topic/beautifulexperiences/\#/about/ (accessed February 28, 2012).

Zomerdijk, Leonieke G., and Christopher A. Voss. 2010. Service design for experiencecentric services. Journal of Service Research 13, no.1: 67-82.

- 2011. NSD processes and practices in experiential services. Journal of Product Innovation Management 28: 63-80. 


\section{APPENDIX A}

Research Overview (Letter of Invitation to Participate and Information)

Garleton

Date: DD/MM/YY

Title of Project: A System's Approach to User Experience Design

Faculty Supervisors:

Wonjoon Chung

School of Industrial Design

wonjoon_chung@carleton.ca

Student Investigators:

Sara Fortier

School of Industrial Design

sfortie2@connect.carleton.ca

Dear Sir or Madame,

My name is Sara Fortier, I am a Master's student in the School of Industrial Design at Carleton University conducting research under the supervision of Dr. WonJoon Chung.

You are invited to participate in a study investigating the design processes involved in total experience design. I have provided an overview of my area of research below for your review. My data collection will consist of a 45 minute phone interview. The interview will be audio recorded. Also, you will not be associated with the data collected.

If you are interested in participating please contact me at:

\section{Sara Fortier}

sfortie2@connect.carleton.ca.

\section{Introduction}

Successful products such as Apple's iPod and Nintendo's Wii have caused an industry wide lust for the illusive "wow" factor; the element that elevates a product, allowing it to outperform its competitors. This has led to a shift in the design discipline away from its traditional focus on a standalone "artifact" and towards a larger system of experiences. Principal to this is the advent of Experience Design or designing for experience; coined by Pine and Gilmour in 1999 as the next frontier in economic offerings.

Fundamental to the industry of services and product development, yet often neglected, is the concept that no product can exist in isolation (Nelson \& Stolterman, 2003), and as such is a part of a larger system of services and products offered within an organization. 
Every component of that system is interrelated and must work together harmoniously, evoking satisfying customer experiences at each stage within the product lifecycle.

\title{
Background
}

At it most basic, an experience is yielded when a person interacts with any element of nature (Dewey, 1934). Thus, every interaction is an experience, whether it is human-toproduct, human-to-environment, or human-to-human. The key to eliciting satisfying experiences is in making each of those interactions pleasurable (Popovic, 2002).

In addition, experiences are innately personal, and thereby evoke different meanings for different people. For this reason, as designers, we cannot design an individual experience. We can only design the elements of interaction through which we can stage the desired experiences (Forlizzi \& Ford, 2000; Sanders, 2001; Pine \& Gilmour, 1999). Moreover, people's experiences are greatly affected by contextual elements within the services environment and the emotional qualities these elements can evoke.

\section{Rationale}

Increasingly, companies are looking to differentiate themselves from their competitors. As the products and services they offer are easily reproduced, the opportunity lies in experience design, an attribute much harder to replicate if done well (Garrett, 2003). While extant literature on experience design has been written from the perspectives of services research, human computer interaction, operational management and hospitality disciplines (Sheng \& Chen, 2011), there are relatively few studies completed from a product design perspective. Yet, central to the concept of experience is the idea that they exist as a result of human interactions with products, not only with services.

\section{Objective}

Through a series of interviews with industry experts in the area of experience design within a system of interconnected products and services I hope to uncover the fundamental components of their services and products that enrich customers experiences. As well as how these lessons can be applied to other businesses and designers.

\section{Expected outcome}

In this study, I hope to develop a set of design principles to aid designers and company visionaries in designing meaningful experiences through their products and services. My research will provide valuable insight into the elements that should be considered when developing innovative products and services to enrich customer experience.

This project has been reviewed and cleared by the Carleton University Research Ethics Board. Should you have any concerns or questions please feel free to contact them at:

\author{
Professor Antonio Gualtieri, Chair \\ Research Ethics Board \\ Carleton University Research Office \\ Carleton University \\ 1125 Colonel By Drive \\ Ottawa, Ontario K1S 5B6
}

Tel: 613-520-2517 E-mail: ethics@carleton.ca 


\section{APPENDIX B}

Interview Script (Including Oral Consent)

Hello,

My name is Sara Fortier, I am a student at Carleton University and I am conducting research for my Master of Design thesis.

Thank you again for taking the time to share your experience with me today. The interview should only take about $\mathbf{4 5}$ minutes, is that still okay with your schedule?

Just to give you a little background on my research; I am looking at the total customer experience, from a broader perspective. Not only user interface, product or service design, but the entire package or system of products working together. Examples of companies that have employed this type of total experience design might be Apple or IKEA. I am most interested in understanding what experience design is for you and your firm, as well as the processes your firm uses to help clients achieve great customer experiences. Your thoughts and comments are all very helpful. Of course, I understand that you may not be able to share some information with me as it may be confidential, but where possible, any examples of what you did in one case or another, great stories, or funny experiences are all excellent.

During the interview, I will be taking notes. To help with data collection, and to help me when I return to my notes I am seeking your consent to audio record this session. Is that okay with you? Also, your name will not be associated with the comments you provide, you may simply be referred to as "Participant X". However, I would like to use your company name in my thesis. Is that alright with you?

Your participation is voluntary and should you wish to skip any questions or end the interview you are free to do so at any time. If for any reason you would like to be removed from the study once the interview has been completed, you may do so at any point, prior to February $30^{\text {th }}, 2012$.

As a final note, I would like to mention that this research has been approved by the Carleton University Research Ethics Board. They can be reached at 613-520-2517, should you have any concerns or questions.

Do you have any questions before we get started?

Great, are you ready to start? 


\section{APPENDIX C}

Interview Protocol

\section{USER PROFILE: [2minutes]}

1. What is your role and job title?

2. What is your professional background in experience design?

a. And how many years have you been doing it for?

\section{COMPANY PROFILE [3minutes]}

3. What is [Company Name]'s client base?

4. What does [Company Name] do for its clients (why do they come to you)?

\section{GENERAL/PROCESS [20 minutes]}

5. What does experience design mean for you at [Company Name] and why is it so important?

6. What is it about the experiences you design for your clients that are so compelling to customers? (You can use an example to explain)

a. What are the most critical components that must be considered when it comes to enhancing the customer experience?

As I mentioned earlier, I am interested in your design process at [Company Name]. I will ask you a few question about that and feel free to add anything I have not asked and you feel to be important or use examples to better explain the concepts.

7. How do you identify the design opportunities that ultimately lead to great customer experiences?

a. What do you need to know first?

b. What are you looking for?

c. What approach do you use when tackling this kind of problem? (Probe: Research Methods)

d. Could you describe your typical design process when it comes to consulting on enhancing a customer experience?

8. What are the most important attributes of a company culture or of their design strategy that one should have if they are looking to design services and products that create great customer experiences? 
9. What is the typical output of your service?

\section{CONCLUSION [3 minutes]}

10. What are some examples of companies offering exceptional customer experiences?

11. What are examples of other experience-centric design agencies/consulting firms?

\section{WRAP-UP [2 minutes]}

That brings me to the end of my questions. Is there anything you would like to add, or any questions you have for me? Would it be alright with you if I have any quick follow-up questions to contact via email? I'll be sure to send you a copy of the results once I have finished all of my interviews.

Thanks again for taking time to help me today. 
APPENDIX D

Units of Analysis (Documents)

\begin{tabular}{|c|c|c|c|c|c|c|}
\hline $\begin{array}{l}\text { Firm/ Unit of } \\
\text { Analysis }\end{array}$ & $\begin{array}{l}\text { About \& } \\
\text { Services }\end{array}$ & Process & $\begin{array}{l}\text { Blog } \\
\text { Post }\end{array}$ & $\begin{array}{l}\text { Case- } \\
\text { Study }\end{array}$ & $\begin{array}{l}\text { White } \\
\text { Paper \& } \\
\text { Articles }\end{array}$ & Other \\
\hline Akendi & $\overline{0}$ & $\square$ & & & 0 & \\
\hline IDEO & (1) & [] & & $\overline{0}$ & $\overline{0}$ & $\overline{0}$ \\
\hline Macadamian & $\bar{\square}$ & & प & & & \\
\hline Adaptive Path & [ & (1) & D & & & \\
\hline Kicker Studio & ( & & & & & \\
\hline Method & ( & & & & $\overline{7}$ & $\overline{0}$ \\
\hline Ziba & $\overline{0}$ & & & & $\overline{0}$ & $\overline{0}$ \\
\hline
\end{tabular}


APPENDIXE

Document Protocol

Case \#:

Medium:

Title:

Date Published:

Author:

Main Topic:

Source:

\section{Brief Description:}

\begin{tabular}{|l|l|l|}
\hline Categories & $\begin{array}{l}\text { Relevant Passages and } \\
\text { Quotations }\end{array}$ & $\begin{array}{l}\text { Researcher } \\
\text { Comments }\end{array}$ \\
\hline $\begin{array}{l}\text { Human Centered Design } \\
\text { (including empathy, in-depth } \\
\text { research and user feedback) }\end{array}$ & & \\
\hline Holistic Thinking & & \\
\hline Harmonizing Touchpoints & & \\
\hline $\begin{array}{l}\text { Interdisciplinary Collaboration } \\
\text { (Including human resource } \\
\text { allocation) }\end{array}$ & & \\
\hline $\begin{array}{l}\text { Client Empowerment (Including } \\
\text { tools and methods) }\end{array}$ & & \\
\hline Storytelling & & \\
\hline
\end{tabular}




\section{APPENDIX F}

Annotated Bibliography - Document Analysis

Adaptive Path. 2011a. In-house training. http://adaptivepath.com/work/services/inhouse-training (accessed February 28, 2012). [descriptions of in-house training workshops provided by Adaptive Path to clients and other designers] 2011b. Services. http://adaptivepath.com/work/services (accessed February 28, 2012). [description on Adaptive Path company website of services they offer, including Experience Strategy, Service Design, Digital Product Design, etc.] 2011c. Work. http://adaptivepath.com/work (accessed February 28, 2012). [description on Adaptive Path company website of work they do and why companies hire them]

Adaptive Path blog. The anatomy of an experience map. http://adaptivepath.com/ideas/the-anatomy-of-an-experience-map (accessed February 28, 2012). [a blog post on the benefits of using and creating experience maps as well as a detailed "how-to" guide]

Akendi. 2012a. Our process. http://www.akendi.ca/end-to-end-experience-designprocess/akendi-experience-design-process.php (accessed February 28, 2012). [description of Akendi's design and research process - referred to as Intentional Experience Creation]

- 2012b. Our services. http://www.akendi.ca/customer-user-experiencedesign/customer-user-experience-research-design-services.php (accessed February 28, 2012). [description on Akendi company website of services they offer] 
Backett, Paul. 2011. Rethinking design education. Ziba Design. Whitepaper. http://www.ziba.com/perspectives/topic/beautifulexperiences/\#/perspectives/a rticle/rethinking-design-education/ (accessed February 28, 2012). [6 part series written for Core77 on rethinking design education: Teach less, integrate more; Research; Sketching; Prototyping; Collaboration; Presentation]

Boulanger, Frederic. 2011. Customer experience and your core business. Macadamian blog, entry posted December 12 . http://www.macadamian.com/blog/post/customer_experience_and_your_core _business/ (accessed February 28, 2012). [blog post describing an unpleasant experience at a hair salon and how your business must master its core offering]

Dust, Fred, and llya Prokopoff. 2009. Designing systems at scale. Rotman Magazine, Winter. [article discussing the issue of wicked problems and the importance for designers to influence people's behaviours on a mass scale in order to impact the larger system]

Farnham, Kevin. n.d. Holistic brand experiences. Method. SWF file. http://method.com/\#/thoughts/people/detail/people/holistic-brandexperiences (accessed February 28, 2012). [short video on building holistic experiences and breaking down silos]

Hately, Matthew. 2011. Google+Motorola=Apple? Puhleeeze. Macadamian blog, entry posted August 18. [blog post describing the Apple experience and how it is the total experience Apple offers, integrated consistently across touchpoints that make it renown] 
Hately, Matthew. 2011. Two ways you can be more innovative. Macadamian blog, entry posted August 25.

http://www.macadamian.com/blog/post/two_ways_you_can_be_more_innovat ive/ (accessed February 28, 2012).

IDEO. 2009. Long-haul travel experience for Air New Zealand.

http://www.ideo.com/work/long-haul-travel-experience/ (accessed February $28,2012)$. [case study of a project IDEO created - the redesign of Air New Zealand airline experience]

_ 2010. Designing the ideal home for "Wounded Warriors" for Clark Realty. http://www.ideo.com/work/designing-the-ideal-home-for-wounded-warriors/ (accessed February 28, 2012). [case study of a project IDEO worked on designed a new model for building accessible homes on military installations]

- 2012. Careers. http://www.ideo.com/contact\#careers (accessed February 28, 2012). [IDEO Careers website - access to many different job postings and desirable qualities in potential candidates]

Macadamian. 2012. Training. http://www.macadamian.com/services/training/ (accessed February 28, 2012). [description of Macadamian's training workshops] 
Murdock, Robert. n.d. How to be a successful designer. Method. SWF file.

http://method.com/\#/thoughts/people/detail/people/how-to-be-a-successfuldesigner (accessed February 28, 2012).

Newbery, Patrick. n.d.(a) Climbing out of the silo. Method. SWF file. http://method.com/\#/thoughts/all/detail/people/climbing-out-of-thesilo (accessed February 28, 2012). [short video describing how to avoid functional silos]

n.d.(b) "Mushrooms": Skill across disciplines. Method. SWF file. http://method.com/\#/thoughts/people/detail/people/mushrooms-skill-acrossdisciplines (accessed February 28, 2012). [short video on cross-disciplinary designers]

Rodriguez, Diego. 2006. "Products and their Ecosystems: Understanding the power of context in product innovation." Core77 Design 2.0: Discussions on Strategy and Innovation. June 6. Mp3

file. http://www.core77.com/development/design2.0/src/Core77_peter_rojas.m p3 (accessed February 28, 2012). [podcast discussing product ecosystems and their contexts - holistic viewpoint]

van Gelderen, Tedde. 2010. Experience lifecycle strategies: Keys to creating an experience-oriented culture. Akendi. Whitepaper. http://www.akendi.ca/endto-end-experience-design-process/akendi-experience-design-process.php (accessed February 28, 2012). [Whitepaper on understand the experience lifecycle and its role in the human experience creation process] Vossoughi, Sohrab. 2010. Designing beautiful experiences. Ziba Design. Whitepaper. www.ziba.com/perspectives/topic/beautifulexperiences/\#/perspectives/article/ 
designing-beautiful-experiences/ (accessed February 28, 2012). [whitepaper discussing the importance of understanding the customer and how Ziba helps their clients to design meaningful experiences for their customer]

\section{Ziba Design. n. d. About.}

http://www.ziba.com/perspectives/topic/beautifulexperiences/\#/about/ (accessed February 28, 2012). [Ziba Design home page - provides an overview of their corporate mission and their professional expertise and backgrounds] 


\title{
APPENDIX G
}

\author{
Sample of Repeating Ideas
}

Repeating Idea \#23: Build cross-functional teams of design generalists (wear multiple hats/take on multiple roles): "they all did kind of everything"

a) "I favour small generalist teams. I mean to put this in opposition to larger, specialist teams, those are two operating models... (P7, p.3)"

b) "We sometimes, depending on the project will have a researcher, but sometimes the design team themselves will do that research (P4 p.3)."

c) "...and my role changes from project to project too (P5 p.4)."

d) "We involve the research and design in a team that everybody sees everything. So the researchers will see the design and put their input and the designers will do some of the visual work and be a note taker in the research so they catch on really quickly what the issues are. I'm a very strong believer in first hand learning. I don't want somebody to make nice research reports and then say, this is what we found and then the designer will say, well I don't really believe that so I'll do the design my way. I believe the design has to be part of the research activity either by doing some of it and be part of it or by virtually being involved in the overall thing and that's very much a philosophy of the company and I'm kind of hung up on that in that I want everyone to wear multiple hats so that they get both the research component and the design component (P6 p.5)."

e) "On the project we then had an additional interaction designer, design researcher, ux person and an intern who would do a lot of work on visual design but they all did kind of everything. It was more the emphases, the researcher would be the one who shot the photos for the storyboards and the interaction designer was involved with the research as well - He was the floater, he was active on both of the two tracks of the project. One of the interaction designers did a lot of trend analysis. This was one of the larger projects, with the manager and four design resources (P7 p.3)."

Repeating Idea \#24: Team involvement in all phases: "You learn so much from the actual experience, it can never be contained on a piece of paper"

a) "I try to avoid the powerpoint thing, I strongly believe in first hand research, the primary research is the most powerful. That is the same thing we use building testing, you see people stumble, there is no substitute for that (P6, p.5)."

b) "you learn so much from the actual experience, it can never be contained on a piece of paper. You need to be there, that alone is so well spent and some of us still have to fight for it, because we wont get it. They'll want one person, and we'll send three, because it will pay back (P6, p.6)."

c) "we extend the philosophy to wanting to build the product and wanting the designers to be involved in building throughout the engineering and vice-versa (P1, p.6)." 
Repeating Idea \#25: Cross-functional teams collaborating tightly from beginning to end (no hand-offs)

a) "a wide range of disciplines, from you know anthropologist to industrral designers to engineers to mechanical engineers to social scientist, whatever, architects (P5 p.3)."

b) "Because it's easier to get a great customer experience when the whole team is collaborating tightly and is aligned on their goals and works side by side throughout the entire project - one of the hardest things to do in creating a great customer experience is if you've gotta hand off designs right - because anything can happen at that part. It's hard right, because even if you're handing off a concept design to a details design team, just imparting all the context for you know why certain decisions were made and not being involved - I think that's one of the biggest destroyers of experience is when it's been designed and developed by completely different people or different agendas (P1, p.7)."

C) "I think a holistic approach, where the interaction design and the visual design and industrial design all work together is really important (P4, p.4)."

d) "We have for example industrial designers here, people with psychology degree, and the design part and we have people with more research backgrounds, my background is in psychology, that's usually how we team up (P6, p.5)."

e) "Everyone on board was sketching storyboards. So again a small mid level crossfunctional team. Ideally everyone was working from the beginning to the end of the project, I got to hand them off, it allows you to flex better, create better, you remember stuff, you get people up to speed (P7, p.3)."

Repeating Idea \#26: Cross-pollinating ideas from the beginning: "Work in this group environment where across different disciplines [...] we all get together and we're exchanging and cross-pollinating our ideas"

a) "they work in this group environment where across different disciplines - and this is the other thing - you don't just have hardcore designers, but you have people who are anthropologists or education background people, or some people who are you know, computer geeks and like we all get together and we're exchanging and crosspollinating our ideas (P5, p.2)."

b) "they do incredible well is that they use without fail cross-functional teams at the early design phases of a project and so they get a lot of idea input, they mix in a lot of personalities (P1, p.9)."

c) "there are a couple of other people who help us to answer those questions around that as a team from the beginning (P2, p.2)."

d) "We actually invite a lot of designers, and a lot of other people and we do a quick but very serious brainstorming session (P2 p.4)." 


\section{APPENDIX H}

Sample of Repeating Ideas Grouped Into Themes

Theme \#1: Every touchpoint counts

R1. Customer experience means every way you touch that product or brand: "anything that a consumer touches that is related to your company or your brand is part of that experience"

R2. Customer experience as a whole is mitigated or enhanced depending on the experience at each touchpoint: "everything that touches the product either adds or takes away from the experience as a whole"

R3. Holistic picture of everyone involved (not just the end user)

Theme \#2: Realizing where you fit

R4. Understanding the user holistically and how each touch point within the chain of events serves them: "A holistic approach to design that takes into account the whole person" - human centered cognitive

R5. Hiring big picture thinkers: "It helps to have big picture thinkers on the team"

R6. Acknowledge other parts of the chain even if you are only designing one channel: "Understanding how that fits in with a larger ecosystem"

R7. Experience unfolds within a wider narrative: "Realizing that it exists within a wider narrative that unfolds when people start to experience a brand"

Theme \#3: Deliver across touchpoints

R8. Broad reaching skill set

R9. A design firm can be successful at experience design when they are able to deliver across touchpoints: "Our ability to design across a whole wide sloth of touchpoints makes us powerful"

R10. Stay involved during the "refinement" stage to help implementation on client's end: "The more we interact at the development level the better execution you get"

R11. Integrate experience consistently across all touchpoints: "I think that consistency is half the battle"

R12. Ensure that you are consistently telling your brand story and how it is tied across touchpoints

R13. Good experience design requires detailed execution of each touchpoint: "Getting into the details of how that design might extrapolate into other screens or products - how those might work across platforms" 
Theme \#4: Understanding the user

R14. Understanding the user; their life, needs, pains, desires

R15. Good experienced design requires understand and serving a user's emotional needs: "When you do good experience design its because you understand your customer's emotional needs and you serve them"

R16. Prioritizing user needs: "You start to really understand what matters and what doesn't"

Theme \#5: Building Empathy

R17. Hire empathetic people: "We focus on hiring people who have this innate sense of empathy"

R18. Empathy is at the heart of everything you do

R19. Have all project members (including client) develop empathy for the user: "When you have empathy you can appreciate what better solutions are inherently"

Theme \#6: In-depth research is the cornerstone of good experience design

R20. In depth research up front forms the foundation of good design: "Preparing and front loading your work is the singular most important step"

R21. Experience design involves in-depth research: ethnography, observations, interviews: "The level of depth we go into is what really differentiates us"

R22. Understanding the context of use is crucial for good experience design: "The cornerstone for everything we do is contextual research and really understanding what context the products and services will be used in" 


\section{APPENDIX $\mid$}

Sample of Themes Grouped Into Theoretical Constructs/Patterns

\section{CLIENT EMPOWERMENT}

Theme \#8: Client involvement

R28. Get the client involved as soon as and as much as possible: "Doing that allows them to empathise"

R29. Clients are no longer "simple receivers of deliverables": "Not to be a simple receiver of deliverables, but to be an active participant in our creation so they understand why decisions were made"

Theme \#9: Client empowerment

R30. Empowering clients to win at design making: "They have to take it [the design] and make it fly"

R31. Empower clients to bring design in-house and maintain it by engendering a sense of ownership: "Experience design needs to become embedded in the clients business because it is their business"

Note: Designing for rich experience [or empowering clients] involves developing compelling and actionable communication artifacts.

Theme \#10: Salient and Compelling deliverables

R32. Make tangible deliverables that the client can take action on: "[Deliverables] should capture the intent of the research findings, communicate them in a way that is concrete, because research findings are pretty abstract"

R33. Stories help everyone understand: "This is compelling and I understand why its compelling because its being presented in a way that I can immediately understand"

R34. To be salient information/data should be represented in a visual way: "We can go through a lot of data and then plot it in a simple way - a way that we can share with clients and get them to understand" 